University of South Carolina

Scholar Commons

1-1992

\title{
A Family of Complexes Associated to an Almost Alternating Map, with Applications to Residual Intersections
}

Andrew R. Kustin

University of South Carolina - Columbia, kustin@math.sc.edu

Bernd Ulrich

Follow this and additional works at: https://scholarcommons.sc.edu/math_facpub

Part of the Mathematics Commons

Publication Info

Memoirs of the American Mathematical Society, Volume 95, Issue 461, 1992, pages 1-95.

(C) 1992 by American Mathematical Society

This Article is brought to you by the Mathematics, Department of at Scholar Commons. It has been accepted for inclusion in Faculty Publications by an authorized administrator of Scholar Commons. For more information, please contact digres@mailbox.sc.edu. 


\title{
A FAMILY OF COMPLEXES ASSOCIATED TO AN ALMOST ALTERNATING MAP, WITH APPLICATIONS TO RESIDUAL INTERSECTIONS
}

\author{
Andrew R. Kustin And Bernd UlRich
}

The notion of residual intersection was introduced by Artin and Nagata [1]; it has been improved and generalized by Huneke [29] and Huneke and Ulrich [32]. Let $I$ and $J$ be prime ideals in a local Gorenstein ring $R$ with ht $I \leq$ ht $J$ and $I$ not contained in $J$. Krull's altitude theorem guarantees that ht $J \leq \mu(I \cap J)$, where $\mu(M)$ is the minimal number of generators of a module $M$. If equality holds, then $J$ is called a geometric residual intersection of $I$. (A more general definition may be found in (10.1).) One example of a residual intersection concerns the ideal $I(X)$, generated by the maximal order minors of a generic matrix $X$. Huneke [29] proved that $I(X)$ is the residual intersection of a height two perfect ideal. Another example demonstrates the ubiquity of residual intersections. Let $I$ be a strongly Cohen-Macaulay $\left(G_{\infty}\right)$ ideal. (Recall that $I$ is a strongly Cohen-Macaulay ideal if the Koszul homology modules of some (and hence any) set of generators of $I$ are Cohen-Macaulay modules; and $I$ satisfies the property $\left(G_{\infty}\right)$ if $\mu\left(I_{P}\right) \leq$ ht $P$ for all prime ideals $P$ of $R$ with $I \subseteq P$.) Consider the extended Rees algebra $R\left[I t, t^{-1}\right]$ of $I$, which is a one-parameter deformation of the associated graded ring $\mathfrak{G r}_{I}(R)=\oplus_{i}\left(I^{i} / I^{i+1}\right)$ of $I$. Huneke has proved that the defining ideal of $R\left[I t, t^{-1}\right]$ is a residual intersection of a hypersurface section on $I$. The first theorem about residual intersections [29, Theorem 3.1] states that if $I$ is a strongly Cohen-Macaulay ideal which satisfies the condition $\left(G_{\infty}\right)$, and $J$ is a geometric residual intersection of $I$, then the ring $R / J$ is Cohen-Macaulay.

When the ideals $I$ and $J$, from the beginning of the preceding paragraph, have the same height, then the notion of residual intersection is the same as the notion of linkage (or liaison) which has proved very useful; see, for example, [3, 14, 23, $31,46,47,48,49]$. (This list of recent papers which use the technique of linkage is far from complete.) One reason that the technique of linkage has been so fruitful is that if $I$ is perfect, then the generators of $J$ and a free resolution of $R / J$ can easily be described in terms of a free resolution of $R / I$. At the present time, there is no comparable result for residual intersections; in fact, if $J$ is a residual intersection of $I$, then very little is known about the resolution of $R / J$ : if the ideal $I$ is a complete intersection, then the generators of $J$ were described in [32] and the resolution of $R / J$ may be found in [7]; if $I$ is a height two perfect ideal, then $J$ is a determinantal ideal (as described above) and the resolution of $R / J$ is given in [20]; if $I$ is a Gorenstein ideal in the linkage class of a complete intersection, and the

Both authors were partially supported by the National Science Foundation 
height of $J$ is precisely ht $I+1$, then the generators of $J$ are described in [41]. The only general result about the resolution of residual intersections concerns the end of the resolution. If $I$ is strongly Cohen-Macaulay and satisfies the condition $\left(G_{\infty}\right)$, or if $I$ is in the linkage class of a complete intersection, then a set of generators for the canonical module of $R / J$ has been described in [32].

The resolution of a residual intersection of $I$ is understood if $I$ is a complete intersection or if $I$ is a perfect height two ideal. The next natural class of ideals to consider is the class of grade three Gorenstein ideals. Let $X$ be a $g \times g$ alternating matrix over $R$ for some odd integer $g$. Suppose that the ideal $I$, generated by the maximal order pfaffians of $X$, has grade three. Let $a_{1}, \ldots, a_{f}$ be elements of $I$, with $f \geq 3$, and let $J$ be the ideal $\left(a_{1}, \ldots, a_{f}\right): I$ of $R$. If $J$ is a proper ideal and ht $J \geq f$, then $J$ is a residual intersection of $I$ (in the more general sense of Definition 10.1). The present paper began as an attempt to answer the questions, "What are the generators of $J$ ?" and "What is the minimal resolution of $R / J ? "$. These questions are answered in Theorem 10.2. In addition, our approach yielded other, unexpected, resolutions. For example, if the grade three Gorenstein ideal $I$ satisfies the condition $\left(G_{\infty}\right)$, then we have resolved $I^{n}$ for all positive integers $n$ (cf. Theorem 6.25). In particular, we calculate depth $\left(R / I^{n}\right)$ for all $n$. (Previously, the resolution of $R / I^{n}$ had been known only for $n=1$ [14] and $n=2$ [4]; and the depth of $R / I^{n}$ had been known only for $n \leq 2$ [22] or $n \geq \mu(I)-2$ [33].) In the "generic" case, we have also resolved "half" of the modules comprising the divisor class group of $R / J$ (cf. Theorem 9.13).

For $X, I$, and $J$ as defined in the beginning of the preceding paragraph, there is a natural first approximation for $J$. The elements $a_{i}$ are in $I$. Let $Y$ be a $g \times f$ matrix which gives the coefficients of the $a_{i}$ with respect to the generators of $I$. We call $\rho=\left[\begin{array}{ll}X & Y\end{array}\right]$ an "almost alternating matrix". The corresponding alternating matrix is

$$
T=\left[\begin{array}{cc}
X & Y \\
-Y^{\mathrm{t}} & 0
\end{array}\right]
$$

Let $J(\rho)$ be the ideal which is generated by the pfaffians of all principal submatrices of $T$ which contain $X$; see (4.10). It is not difficult to show that $J(\rho)$ is contained in $J$ and that both ideals have the same grade. (See, for example, [42].) Instead of proving directly that $J(\rho)$ is all of $J$, we went straight to our second task; namely, we worked out the entire free $R$-resolution of $R / J$. This can be done without prior knowledge of the generators of $J$, since $J$ is perfect and the canonical module $\omega$ of $R / J$ is known from [32]. In fact, it turns out that $\omega$ is the symmetric power $S_{f-2}(\operatorname{coker} \rho)$ of the cokernel of $\rho$. Thus, we will have proved the equality $J=J(\rho)$ and constructed a resolution of $R / J$, once we are able to resolve symmetric powers of the cokernel of a (generic) almost alternating matrix. Since we resolve all symmetric powers of coker $\rho$, we obtain, in addition, resolutions for "half of the divisor class group" of $R / J$, at least in the generic case.

Now, if we view $\rho: E^{*} \rightarrow G$ as an almost alternating map of free $R$-modules (see Definition 2.1), then the zeroth homology of the Koszul complex

$$
\bigwedge_{S \bullet G}\left(E^{*} \otimes_{R} S_{\bullet} G\right)=S_{\bullet} G \otimes_{R} \grave{\bullet} E^{*}
$$


is $S_{\bullet}(\operatorname{coker} \rho)$. The graded strand

$$
0 \rightarrow S_{0} G \otimes \bigwedge^{i} E^{*} \rightarrow S_{1} G \otimes \bigwedge^{i-1} E^{*} \rightarrow \cdots \rightarrow S_{i-1} G \otimes \bigwedge^{1} E^{*} \rightarrow S_{i} G \otimes \bigwedge^{0} E^{*} \rightarrow 0
$$

of $S_{\bullet} G \otimes_{R} \wedge^{\bullet} E^{*}$ is not acyclic; but, its zeroth homology is equal to $S_{i}(\operatorname{coker} \rho)$. At this point, we are reminded of the classical family of complexes, $\left\{\mathfrak{C}^{i}\right\}$, which is associated to a generic map $\rho: E^{*} \rightarrow G$. The complexes $\mathfrak{C}^{0}$ and $\mathfrak{C}^{1}$ are known as the Eagon-Northcott complex and the Buchsbaum-Rim complex, respectively. There does not seem to be a name for the entire family $\left\{\mathfrak{C}^{i}\right\}$; nonetheless, it has been considered often by many authors. (See, for example, $[20,10,15,12,37,8$, 9].) If $\rho$ is a generic map, then the resolution $\mathfrak{C}^{i}$ of $S_{i}(\operatorname{coker} \rho)$ is

$$
\begin{gathered}
\cdots \rightarrow D_{2} G^{*} \otimes \bigwedge^{g+i+2} E^{*} \rightarrow D_{1} G^{*} \otimes \bigwedge^{g+i+1} E^{*} \rightarrow D_{0} G^{*} \otimes \bigwedge^{g+i} E^{*} \rightarrow S_{0} G \otimes \bigwedge^{i} E^{*} \\
\rightarrow S_{1} G \otimes \bigwedge^{i-1} E^{*} \rightarrow S_{2} G \otimes \bigwedge^{i-2} E^{*} \rightarrow \ldots
\end{gathered}
$$

with $S_{0} G \otimes \bigwedge^{i} E^{*}$ in position $i$. The maps $S_{a} G \otimes \bigwedge^{b} E^{*} \rightarrow S_{a+1} G \otimes \bigwedge^{b-1} E^{*}$ are Koszul maps; the maps $D_{a} G^{*} \otimes \bigwedge^{b} E^{*} \rightarrow D_{a-1} G^{*} \otimes \bigwedge^{b-1} E^{*}$ are Eagon-Northcott maps; and the map

$$
D_{0} G^{*} \otimes \bigwedge^{g+i} E^{*} \rightarrow S_{0} G \otimes \bigwedge^{i} E^{*}
$$

is the composition

$$
\bigwedge^{g+i} E^{*} \stackrel{\Delta}{\longrightarrow} \bigwedge^{g} E^{*} \otimes \bigwedge^{i} E^{*} \stackrel{\bigwedge^{g} \rho \otimes 1}{\longrightarrow} \bigwedge^{g} G \otimes \bigwedge^{i} E^{*} \stackrel{[] \otimes 1}{\longrightarrow} \bigwedge^{i} E^{*}
$$

where $\Delta$ is co-multiplication, $g=\operatorname{rank} G$, and [ ] is orientation. (See section one for more details.) By using the family $\left\{\mathfrak{C}^{i}\right\}$ as a model we were able to produce a family of complexes $\left\{\mathfrak{D}^{q}\right\}$ with the property that if $\rho$ is a "sufficiently general" almost alternating map, then $\mathfrak{D}^{q}$ is a resolution of $S_{q}(\operatorname{coker} \rho)$ for all $q \geq 1$. The family of complexes $\left\{\mathfrak{D}^{q}\right\}$ has many properties which are analogous to the family $\left\{\mathfrak{C}^{i}\right\}$.

If we are willing to omit all details, then the family of complexes $\left\{\mathfrak{D}^{q}\right\}$ can be described very quickly. The right side of $\mathfrak{D}^{q}$, see (2.7), is a graded strand of the quotient

$$
K_{\bullet \bullet}=\frac{S_{\bullet} G \otimes \Lambda^{\bullet} E^{*}}{\mathcal{J}}
$$

where $S_{\bullet} G \otimes \bigwedge^{\bullet} E^{*}$ is the Koszul algebra mentioned above and $\mathcal{J}$ is the differential graded ideal generated by the two elements $\eta$ and $\lambda$ of (2.13). The left side of $\mathfrak{D}^{q}$ is the dual of the right side of $\mathfrak{D}^{f-2-q}$. Sitting between these two sides is a module we have called $Q_{q}$ (see 2.16). All of the maps in $\mathfrak{D}^{q}$ are defined above and are linear except for two:

$$
\left(K_{0 f-q-2}\right)^{*} \rightarrow Q_{q} \rightarrow K_{0 q}
$$


The map $Q_{q} \rightarrow K_{0 q}$ is defined in (2.15 f); it involves pfaffians of all possible sizes and lives in many degrees. Up to sign, the map $\left(K_{0 f-q-2}\right)^{*} \rightarrow Q_{q}$ is the dual of $Q_{f-q-2} \rightarrow K_{0 f-q-2}$.

Our interest in the complexes $\left\{\mathfrak{D}^{q}\right\}$ was motivated by the notion of residual intersection; however, residual intersections play no role in our construction of the family $\left\{\mathfrak{D}^{q}\right\}$ or in our proof of the properties of these complexes. After the present paragraph, the next serious consideration of residual intersections takes place in section 10. In sections 1 through $9, R$ is an arbitrary commutative noetherian ring and $\rho$ is an almost alternating map of shape $(g, f)$ where $g \geq 1$ and $f \geq 0$. (In other

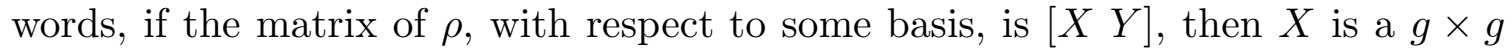
alternating matrix and $Y$ is a $g \times f$ matrix.) Notice that we do not require $f$ to be at least three, as we would if we were solely dealing with residual intersections. In fact, when we take $f$ to be zero, we obtain, in Theorem 6.25 , the resolution of the powers of a grade three Gorenstein ideal. This result, although one of the main theorems in the paper, comes more or less for free given the basic strategy of the paper. Also, we do not require $g$ to be odd, as we would if we insisted that the maximal order pfaffians of $X$ generate a grade three Gorenstein ideal. This flexibility allows us to describe, in Theorem 10.2, the minimal resolution of all residual intersections of a grade three Gorenstein ideal in a Gorenstein local ring $(R, \mathfrak{m})$, not merely those residual intersections of the form $J=(A: I)$ with $A \subseteq \mathfrak{m} I$.

We conclude the introduction by describing the contents of each section. Section one contains all of the relevant background material about multilinear algebra, pfaffians, commutative algebra, and index sets. Most readers will probably want to refer to this section only when necessary. In section two we define $\mathfrak{D}^{q}$ and we prove that $\mathfrak{D}^{q}$ is a complex, except possibly at $Q_{q}$. We also give an alternate description for various parts of $\mathfrak{D}^{q}$. In section three we complete the proof that $\mathfrak{D}^{q}$ is a complex by showing that $\mathfrak{D}^{q}$ is a complex at $Q_{q}$. There is nothing analogous to this step in the construction of the family $\left\{\mathfrak{C}^{i}\right\}$. The proof in section three is based on the pfaffian identity we have labeled (3.2). It is possible that this identity may be of independent interest. We have noticed that Srinivasan [50] used a similar identity when she put a differential graded algebra structure [51] on the minimal resolution of the deviation two Huneke-Ulrich Gorenstein ideals. In addition, Day [19] has used a similar identity in his proof that a polynomial ring is an Algebra with Straightening Law on the poset of all pfaffians of a generic alternating matrix. At any rate, we prove identity (3.2) by formulating it in a coordinate-free manner and then picking the most convenient basis. Eventually, the problem is reduced to a combinatorial lemma (Lemma 3.12) about permutations of sets of integers.

Section four, "Elementary facts about the complexes $\left\{\mathfrak{D}^{q}\right\}$ ", is relatively easy reading. We begin by giving a portrait of the family $\left\{\mathfrak{D}^{q}\right\}$. This will be many reader's favorite part of the paper. In Proposition 4.13, we record the homology $H_{0}\left(\mathfrak{D}^{q}\right)$ for $q \geq 0$. Proposition 4.23 contains the long exact sequence of homology which we use in our inductive proof of the acyclicity of the complexes $\mathfrak{D}^{q}$. The section concludes with a record of the degrees of the maps in $\mathfrak{D}^{q}$. The ultimate theorems about the acyclicity of the complexes $\mathfrak{D}^{q}$ (Theorems 6.17 and 8.3) state that if $\rho$ is a "sufficiently general" almost alternating map, then $\mathfrak{D}^{q}$ is an acyclic complex for all $q \geq-1$. Section five contains our precise formulation of "sufficiently general". It also contains some relatively technical calculations of lower bounds for 
the grade of all of the relevant ideals. In section six we prove that the complexes $\mathfrak{D}^{q}$ are acyclic in the case that $f=0$. In this case, the almost alternating matrix $\rho=\left[\begin{array}{ll}X & Y\end{array}\right]$ is simply the alternating matrix $X$. Our proof is an application of the Buchsbaum-Eisenbud criterion [11]. We conclude the section by observing that, when $g$ is odd, the $\mathfrak{D}^{q}$ are resolutions of powers of the grade three Gorenstein ideal $\operatorname{Pf}_{g-1}(X)$.

In section seven we prove, by induction on $f$, that if $\rho$ is a generic almost alternating matrix, then the complex $\mathfrak{D}^{q}$ is acyclic for $q \geq-1$. In section eight, we specialize the result of section seven to the case where $\rho$ is merely "sufficiently general". In particular, we see that if the ideal $J(\rho)$ has grade at least $f$, then it is perfect of grade $f$. In Corollary 8.9 we make the calculation which shows that $J(\rho)$ is the residual intersection of $\operatorname{Pf}_{g-1}(X)$. In section nine we emphasize the determinantal-like nature of the ideals $J(\rho)$ when $\rho$ is a generic almost alternating matrix. Let $\bar{R}=R_{0}[X, Y] / J(\rho)$. The ring $\bar{R}$ is reduced (or a domain or normal) if and only if the ring $R_{0}$ has the same property. We also give a lower bound for the codimension of the singular locus of $\bar{R}$ in terms of the codimension of the singular locus of $R_{0}$. If $R_{0}$ is a normal domain, then we calculate the divisor class group of $\bar{R}$ in terms of the divisor class group of $R_{0}$. In section ten we interpret the complexes $\left\{\mathfrak{D}^{q}\right\}$ in terms of residual intersections. 


\section{Section 1. Preliminary Concepts.}

Fix a commutative noetherian ring $R$. All $R$-modules that we consider are finitely generated. If $M$ is an $R$-module, then $M^{*}$ is the dual, $\operatorname{Hom}_{R}(M, R)$, of $M$ and

$$
\langle,\rangle: M \otimes M^{*} \rightarrow R \text { and }\langle,\rangle: M^{*} \otimes M \rightarrow R
$$

each represent the evaluation map. Let $E$ be a free $R$-module of rank $e$. We will make much use of the symmetric algebra $S(E)$, the exterior algebra $\bigwedge(E)$, and the divided power algebra $D(E)$. (The formal properties of these algebras may be found in [13].) Each of these algebras $A$ comes equipped with a multiplication $\mu: A \otimes A \rightarrow A$, and a co-multiplication $\Delta: A \rightarrow A \otimes A$. The co-multiplication is induced by the diagonal map $E \rightarrow E \oplus E$. For each non-negative integer $i$, the co-multiplication maps together with the evaluation map $\langle, \quad\rangle: E \otimes E^{*} \rightarrow R$ induce canonical perfect pairings

$$
\begin{aligned}
& \langle\langle,\rangle\rangle: \bigwedge^{i} E \otimes \bigwedge^{i} E^{*} \rightarrow R, \quad \text { and } \\
& \langle\langle,\rangle\rangle: D_{i}(E) \otimes S_{i}\left(E^{*}\right) \rightarrow R .
\end{aligned}
$$

In particular, if $x_{1}, \ldots, x_{i} \in E$ and $y_{1}, \ldots, y_{i} \in E^{*}$, then the value of

$$
\left\langle\left\langle x_{1} \wedge \ldots \wedge x_{i}, y_{1} \wedge \ldots \wedge y_{i}\right\rangle\right\rangle
$$

under the map of (1.2) is the determinant of the $i \times i$ matrix with $\left\langle x_{a}, y_{b}\right\rangle$ in row $\mathrm{a}$ and column $\mathrm{b}$. The map of (1.3) is defined so that

$$
\left\langle\left\langle x_{1}^{(i)}, y_{1} y_{2} \ldots y_{i}\right\rangle\right\rangle=\left\langle x_{1}, y_{1}\right\rangle\left\langle x_{1}, y_{2}\right\rangle \ldots\left\langle x_{1}, y_{i}\right\rangle
$$

and so that the diagram

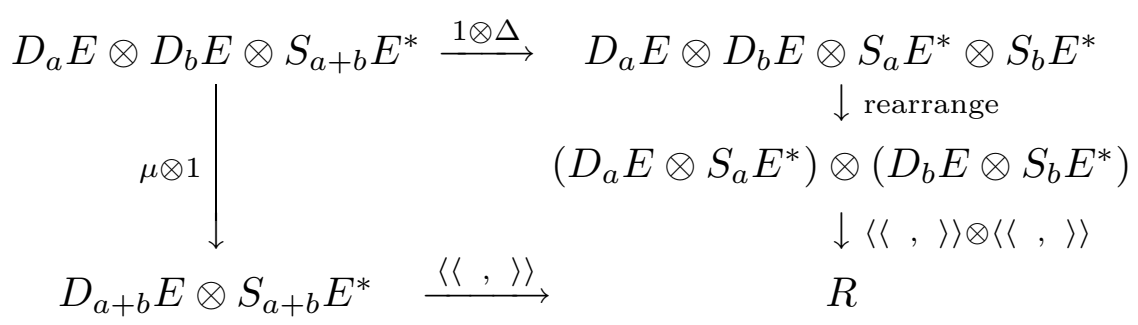

commutes. Both of these pairings have a particularly attractive form when expressed in terms of bases. Let $\varepsilon_{1}, \ldots, \varepsilon_{e}$ be any basis for $E$ and let $\varepsilon_{1}^{*}, \ldots, \varepsilon_{e}^{*}$ be the corresponding dual basis for $E^{*}$. If $a_{1}, \ldots, a_{e}$ and $b_{1}, \ldots, b_{e}$ are non-negative integers with $\sum a_{j}=\sum b_{j}=i$, then

$$
x=\varepsilon_{1}^{\left(a_{1}\right)} \ldots \varepsilon_{e}^{\left(a_{e}\right)} \quad \text { and } \quad y=\left(\varepsilon_{1}^{*}\right)^{b_{1}} \ldots\left(\varepsilon_{e}^{*}\right)^{b_{e}}
$$


are elements of $D_{i}(E)$, and $S_{i}\left(E^{*}\right)$, respectively. When the pairing of (1.3) is applied to $x \otimes y$, we obtain $\langle\langle x, y\rangle\rangle=1$, if $a_{j}=b_{j}$ for all $j$; and $\langle\langle x, y\rangle\rangle=0$, otherwise. Similarly, if $a_{1}, \ldots, a_{i}, b_{1}, \ldots, b_{i}$ are integers with

$$
1 \leq a_{1}<a_{2} \ldots<a_{i} \leq e, \quad \text { and } \quad 1 \leq b_{1}<b_{2}<\ldots<b_{i} \leq e
$$

then

$$
x=\varepsilon_{a_{1}} \wedge \ldots \wedge \varepsilon_{a_{i}} \in \bigwedge^{i} E, \text { and } y=\varepsilon_{b_{1}}^{*} \wedge \ldots \wedge \varepsilon_{b_{i}}^{*} \in \bigwedge^{i} E^{*}
$$

When the pairing of (1.2) is applied to $x \otimes y$ we obtain $\langle\langle x, y\rangle\rangle=1$ if $a_{j}=b_{j}$ for all $j$, and $\langle\langle x, y\rangle\rangle=0$ otherwise.

Every finitely generated free $R$-module, that we consider, comes equipped with an orientation. That is, there are fixed isomorphisms

$$
[]: \bigwedge^{e} E \rightarrow R \text { and }[]: \bigwedge^{e} E^{*} \rightarrow R
$$

which are compatible with the pairing of (1.2) in the sense that

$$
\langle\langle x, y\rangle\rangle=[x][y]
$$

for $x \in \bigwedge^{e} E$ and $y \in \bigwedge^{e} E^{*}$. Every basis $\varepsilon_{1}, \ldots, \varepsilon_{e}$ that we consider for $E$ has the property that

$$
\left[\varepsilon_{1} \wedge \ldots \wedge \varepsilon_{e}\right]=1
$$

Exterior multiplication, followed by the orientation isomorphism, gives a canonical perfect pairing

$$
\bigwedge^{i} E \otimes \bigwedge^{e-i} E \rightarrow R
$$

For each integer $i$, the canonical perfect pairings of (1.2) and (1.5) induce an isomorphism

$$
\beta: \bigwedge^{e-i} E \rightarrow \bigwedge^{i} E^{*}
$$

which is defined by

$$
[x \wedge y]=\langle\langle x, \beta(y)\rangle\rangle
$$

for all $x \in \bigwedge^{i} E$ and $y \in \bigwedge^{e-i} E$, where []$: \bigwedge^{e} E \rightarrow R$ is the orientation isomorphism of (1.4) and $\langle\langle\rangle$,$\rangle is the pairing of (1.2). Let \varepsilon_{1}, \ldots, \varepsilon_{e}$ be a basis for $E$ and $\varepsilon_{1}^{*}, \ldots, \varepsilon_{e}^{*}$ be the corresponding dual basis for $E^{*}$. If

$$
\left\{a_{1}, \ldots, a_{e-i}\right\} \cup\left\{b_{1}, \ldots, b_{i}\right\}=\{1, \ldots, e\},
$$

then

$$
\beta\left(\varepsilon_{a_{1}} \wedge \ldots \wedge \varepsilon_{a_{e-i}}\right)= \pm \varepsilon_{b_{1}}^{*} \wedge \ldots \wedge \varepsilon_{b_{i}}^{*}
$$


where the sign if determined by (1.7).

Let $\psi: F \rightarrow G$ be a map of free $R$-modules. Throughout this paper we will make much use of two differential $R$-algebras associated to $\psi$. The Koszul algebra associated to $\psi$ is the $R$-algebra

$$
\left(S_{\bullet} G \otimes \dot{\wedge} F, \partial\right)
$$

where the differential $\partial: S_{a} G \otimes \bigwedge^{b} F \rightarrow S_{a+1} G \otimes \bigwedge^{b-1} F$ is the composition

$$
S_{a} G \otimes \bigwedge^{b} F \stackrel{1 \otimes \Delta}{\longrightarrow} S_{a} G \otimes F \otimes \bigwedge^{b-1} F \stackrel{1 \otimes \psi \otimes 1}{\longrightarrow} S_{a} G \otimes G \otimes \bigwedge^{b-1} F \stackrel{\mu \otimes 1}{\longrightarrow} S_{a+1} G \otimes \bigwedge^{b-1} F .
$$

(Here and in the remainder of the paper, we use the convention that

$$
S_{a} G \cong D_{a} G \cong \bigwedge^{a} G=0
$$

if $a<0$.) Observe that the Koszul algebra of (1.8) is canonically isomorphic to the exterior algebra

$$
\grave{\bigwedge}_{S}\left(S \otimes_{R} F\right)
$$

(where $S$ is the symmetric algebra $S_{\bullet} G$ ); and consequently, it is a differential graded-commutative algebra. In fact, the algebra of (1.8) is the "usual Koszul complex" which is associated to the map

$$
S \otimes_{R} F \stackrel{1 \otimes \psi}{\longrightarrow} S \otimes_{R} G \stackrel{\text { mult }}{\longrightarrow} S
$$

from the free, rank $f, S$-module $S \otimes_{R} F$ to $S$. Following the lead of [7] we say that

$$
\left(D \cdot G^{*} \otimes \dot{\bigwedge} F, \delta\right)
$$

is the Eagon-Northcott algebra associated to $\psi$. The differential

$$
\delta: D_{a} G^{*} \otimes \bigwedge^{b} F \rightarrow D_{a-1} G^{*} \otimes \bigwedge^{b-1} F
$$

is the composition:

$$
\begin{gathered}
D_{a} G^{*} \otimes \bigwedge^{b} F \stackrel{\Delta \otimes \Delta}{\longrightarrow} D_{a-1} G^{*} \otimes G^{*} \otimes F \otimes \bigwedge^{b-1} F \stackrel{1 \otimes 1 \otimes \psi \otimes 1}{\longrightarrow} \\
D_{a-1} G^{*} \otimes G^{*} \otimes G \otimes \bigwedge^{b-1} F \stackrel{1 \otimes<,, \gg 1}{\longrightarrow} D_{a-1} G^{*} \otimes \bigwedge^{b-1} F .
\end{gathered}
$$


Observation 1.13. Let $\psi: F \rightarrow G$ be a map of free $R$-modules. The graded strands of the Koszul algebra associated to $\psi$ are dual to the graded strands of the Eagon-Northcott algebra associated to $\psi$.

Proof. Let $f$ be the rank of $F$. The perfect pairings of (1.3) and (1.5) give a perfect pairing

$$
\langle\langle,\rangle\rangle:\left(D_{a} G^{*} \otimes \bigwedge^{b} F\right) \otimes\left(S_{a} G \otimes \bigwedge^{f-b} F\right) \rightarrow R
$$

A straightforward calculation shows that if $z \in S_{a} G \otimes \bigwedge^{f-b} F$ and $y \in D_{a+1} G^{*} \otimes$ $\bigwedge^{b+1} F$, then

$$
\langle\langle\delta y, z\rangle\rangle=(-1)^{b}\langle\langle y, \partial z\rangle\rangle .
$$

Thus $\langle\langle\rangle$,$\rangle induces the following isomorphism of complexes:$

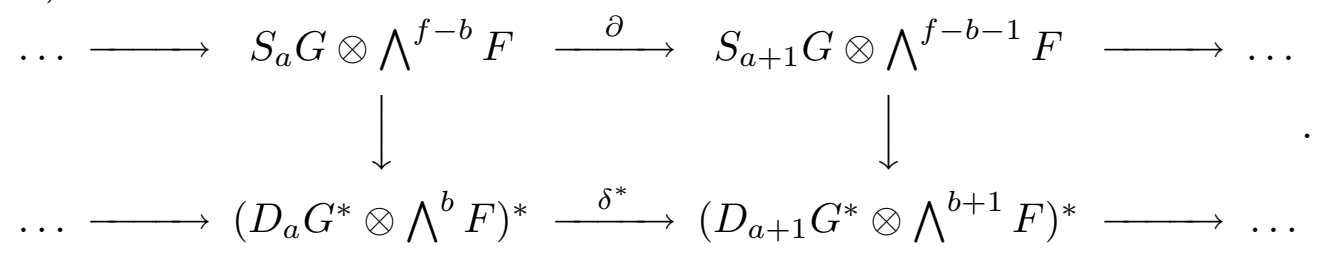

Definition 1.17. If $G$ is a free $R$-module, then the homomorphism $\xi: G^{*} \rightarrow G$ is called alternating if

$$
\left\langle\xi\left(\Gamma_{1}\right), \Gamma_{2}\right\rangle+\left\langle\xi\left(\Gamma_{2}\right), \Gamma_{1}\right\rangle=0 \quad \text { and } \quad\langle\xi(\Gamma), \Gamma\rangle=0
$$

for all elements $\Gamma_{1}, \Gamma_{2}$, and $\Gamma$ and $G^{*}$. (The map $\langle, \quad\rangle: G \otimes G^{*} \rightarrow R$ is the evaluation map of (1.1).)

In particular, if $\gamma_{1}, \ldots, \gamma_{g}$ is a basis for $G$ and $\gamma_{1}^{*}, \ldots, \gamma_{g}^{*}$ is the corresponding dual basis for $G^{*}$, then the matrix $X=\left(x_{i j}\right)$ of the alternating map $\xi: G^{*} \rightarrow G$ with respect to these bases is an alternating matrix. That is, $x_{j i}=-x_{i j}$ and $x_{i i}=0$ for all $i$ and $j$. The pfaffian of $X, \operatorname{pf}(X)$, is an element of $R$ whose square is the determinant of $X$. The pfaffian of $X$ is zero if $g$ is odd. For even sized matrices the pfaffian is defined by the Laplace expansion together with the convention

$$
\operatorname{pf}\left[\begin{array}{cc}
0 & x_{12} \\
-x_{12} & 0
\end{array}\right]=x_{12} \text {. }
$$

For integers $i$ and $j$, let

$$
\sigma(i j)= \begin{cases}1 & \text { if } i<j \\ 0 & \text { if } i=j \\ -1 & \text { if } j<i\end{cases}
$$

and let $X(i j)$ be $\sigma(i j)$ times the pfaffian of $X$ with rows and columns $i$ and $j$ removed. The Laplace expansion for pfaffians is:

$$
\sum_{j}(-1)^{j} x_{i j} X(k j)=(-1)^{i+1} \delta_{i k} \operatorname{pf}(X) .
$$


If $\xi: G^{*} \rightarrow G$ is an alternating homomorphism, then we will make much use of the divided powers $\xi^{(p)}: \bigwedge^{2 p} G^{*} \rightarrow R$ of $\xi$. The homomorphism $\xi$ induces an alternating bilinear form $\xi^{(1)}: \bigwedge^{2} G^{*} \rightarrow R$ with $\xi^{(1)}\left(\Gamma_{1} \wedge \Gamma_{2}\right)=\left\langle\xi \Gamma_{1}, \Gamma_{2}\right\rangle$ for all $\Gamma_{1}$ and $\Gamma_{2}$ in $G^{*}$. Let $\Xi$ be the element of $\bigwedge^{2} G$ which corresponds to $\xi^{(1)} \in\left(\bigwedge^{2} G^{*}\right)^{*}$ under the canonical isomorphism induced by (1.2). The exterior algebra $\Lambda^{\bullet} G$ is an algebra with divided powers (see, for example, [13, pg. 258]); consequently, $\Xi^{(p)}$ is a well defined element of $\bigwedge^{2 p} G$. Let $\xi^{(p)}: \bigwedge^{2 p} G^{*} \rightarrow R$ be the corresponding element of $\left(\bigwedge^{2 p} G^{*}\right)^{*}$.

Observation 1.19. Let $G$ be a free $R$-module; $\xi: G^{*} \rightarrow G$ be an alternating map and $\Gamma_{1}, \ldots, \Gamma_{2 p}$ be arbitrary elements of $G^{*}$. Then the map $\xi^{(p)}: \wedge^{2 p} G^{*} \rightarrow R$ has been defined so that $\xi^{(p)}\left(\Gamma_{1} \wedge \ldots \wedge \Gamma_{2 p}\right)$ is equal to the pfaffian of the $2 p \times 2 p$ matrix with $\left\langle\xi \Gamma_{i}, \Gamma_{j}\right\rangle$ in row $i$ and column $j$.

By combining the pfaffian expansion formula of (1.18) with the explicit realization of the divided power $\xi^{(p)}$ in Observation 1.19 we obtain the following commutative diagram:

$$
\begin{array}{ccc}
G^{*} \otimes \bigwedge^{2 p-1} G^{*} & \multicolumn{1}{c}{} & \bigwedge^{2 p} G^{*} \\
1 \otimes \Delta \downarrow & & \downarrow \xi^{(p)} \\
G^{*} \otimes G^{*} \otimes \bigwedge^{2 p-2} G^{*} \stackrel{\mu \otimes 1}{\longrightarrow} \bigwedge^{2} G^{*} \otimes \bigwedge^{2 p-2} G^{*} \stackrel{\xi^{(1)} \otimes \xi^{(p-1)}}{\longrightarrow} & R .
\end{array}
$$

In other words, $\xi^{(p)}\left(\Gamma \wedge \Gamma_{1} \wedge \ldots \wedge \Gamma_{2 p-1}\right)$ is equal to

$$
\sum_{i=1}^{2 p-1}(-1)^{i+1}\left\langle\xi \Gamma, \Gamma_{i}\right\rangle \xi^{(p-1)}\left(\Gamma_{1} \wedge \ldots \wedge \widehat{\Gamma}_{i} \wedge \ldots \wedge \Gamma_{2 p-1}\right)
$$

for all integers $p \geq 1$, and for all $\Gamma, \Gamma_{1}, \ldots, \Gamma_{2 p-1}$ in $G^{*}$.

Many of the results we prove are valid over any commutative noetherian ring (not necessarily graded, local, or Cohen-Macaulay); consequently, we are obliged to define a few of the concepts which are used throughout the paper. One reference which uses terms very much the same way we do is the book by Bruns and Vetter [9]. The ideal $I$ of the ring $R$ is unmixed of height $h$ if ht $P=h$ for all prime ideals $P$ of $R$ with $P \in \operatorname{Ass}(R / I)$. The proper ideal $I$ of $R$ has grade $g$ if the longest regular $R$-sequence in $I$ consists of $g$ elements. (We use the phrase "grade $I \geq n$ " to mean, either, that $I$ is a proper ideal of grade at least $n$, or else, that $I$ is the entire ring $R$.) Let $M \neq 0$ be an $R$-module. (As always, $M$ is finitely generated.) The projective dimension of $M$ is denoted by $\operatorname{pd}_{R} M$ and the annihilator of $M$ is denoted ann $M$. The inequality

$$
\operatorname{grade}(\operatorname{ann} M) \leq \operatorname{pd}_{R} M
$$

always holds. If equality holds in (1.21), then $M$ is called a perfect $R$-module. The ideal $I$ in $R$ is perfect if $R / I$ is a perfect $R$-module. It is an easy exercise (see, for example, [9, Proposition 16.19]) to verify that if $M$ is a perfect $R$-module and $R$ is a Cohen-Macaulay ring, then $M$ is a Cohen-Macaulay $R$-module; that is, depth $M_{P}=\operatorname{dim} M_{P}$ for all prime ideals $P$ in the support of $M$. In particular, if $I$ is a perfect ideal in a Cohen-Macaulay ring $R$, then $R / I$ is also a Cohen-Macaulay ring. The following result of Eagon and Northcott may be found in [25, Proposition 6.14] or [9, Theorem 3.15]. 
Proposition 1.22. Let $R \rightarrow S$ be a homomorphism of commutative noetherian rings, and let $\mathbb{F}$ be a projective resolution of a perfect $R$-module $M$. If grade (ann $M) S \geq$ $\operatorname{pd}_{R} M$, then $\mathbb{F} \otimes S$ is acyclic. Furthermore, if $(\operatorname{ann} M) S \neq S$, then $M \otimes S$ is a perfect $S$-module and $\operatorname{pd}_{R} M=\operatorname{pd}_{S}(M \otimes S)=\operatorname{grade}(\operatorname{ann} M) S$.

Some of the most important examples of perfect ideals are the determinantal ideals. Let $\psi: F \rightarrow G$ be a map of free $R$-modules with $\operatorname{rank} F=f \geq g=$ $\operatorname{rank} G \geq 1$. Let $Y$ be the matrix of $\psi$ with respect to some bases for $F$ and $G$. If $t$ is a positive integer, then $I_{t}(\psi)$ is the ideal in $R$ generated by the $t \times t$ minors of $Y$. This ideal is independent of the choice of bases for $F$ and $G$. (We let $I_{0}(\psi)$ represent all of the ring $R$.) The rank of $\psi$ is $t$ if $I_{t}(\psi) \neq 0$, but $I_{t+1}(\psi)=0$. We write $I(\psi)$ to mean $I_{t}(\psi)$ for $t=\operatorname{rank}(\psi)$. Now suppose that $\psi$ is a generic map. That is, suppose that there is a commutative noetherian ring $R_{0}$, and that there are bases for $F$ and $G$ so that the entries, $y_{i j}$, of the matrix of $\psi$ with respect to these bases are indeterminates over $R_{0}$ with $R=R_{0}\left[\left\{y_{i j}\right\}\right]$. Hochster and Eagon [26] have proved that for each integer $t$, with $1 \leq t \leq g$, the ideal $I_{t}(\psi)$ of $R$ is perfect with grade equal to

$$
(f-t+1)(g-t+1) .
$$

Ideals generated by the pfaffians of a generic alternating map are also perfect. Let $G$ be a free module of rank $g \geq 1, \xi: G^{*} \rightarrow G$ be an alternating map, and $X=\left(x_{i j}\right)$ be the alternating matrix which represents $\xi$ with respect to some basis for $G$ as described after Definition 1.17. If $t$ is a positive even integer, then $\operatorname{Pf}_{t}(\xi)$ is the ideal generated by the pfaffians of all principal $t \times t$ submatrices of $X$. This ideal is independent of the choice of basis for $G$. (We let $\operatorname{Pf}_{0}(\xi)$ represent all of the ring $R$.) The map $\xi$ is called a generic alternating map if the entries $x_{i j}$ of $X$ with $1 \leq i<j \leq g$ are indeterminates over some subring $R_{0}$ of $R$ and $R$ is the polynomial ring $R_{0}\left[\left\{x_{i j} \mid 1 \leq i<j \leq g\right\}\right]$. If $\xi$ is a generic alternating map, then for every even integer $t$, with $2 \leq t \leq g$, the ideal $\mathrm{Pf}_{t}(\xi)$ is perfect of grade

$$
\left(\begin{array}{c}
g-t+2 \\
2
\end{array}\right) .
$$

This last fact has been proved by Marinov [43, 44] and Kleppe and Laksov [38] using the principal radical systems technique of Hochster and Eagon. A combinatorial proof which is valid when $R$ contains the rational numbers may be found in [35].

Let $M$ be a finitely generated $R$-module. We say that $M$ is torsion-free if every non-zero-divisor in $R$ is regular on $M$; we say that $M$ is reflexive if the natural map $M \rightarrow M^{* *}$ is an isomorphism. The following Serre type conditions provide a way to prove that a particular module is torsion-free or reflexive. Following the lead of [9, Proposition 16.29] we say that the $R$-module $M$ satisfies the condition $\left(\widetilde{S}_{n}\right)$ if

$$
\operatorname{depth} M_{P} \geq \min \left\{n \text {, depth } R_{P}\right\} \text { for all } P \in \operatorname{Spec} R \text {. }
$$

(The usual Serre conditions $\left(S_{k}\right)$ and $\left(R_{k}\right)$ may be found in [43, (17.I)].) If the ring $R$ satisfies the usual Serre condition $\left(S_{n}\right)$ and $k \leq n$, then the $R$-module $M$ satisfies $\left(\widetilde{S}_{k}\right)$ if and only if it satisfies $\left(S_{k}\right)$. In particular, if $R$ is a Cohen-Macaulay 
ring, then $\left(\widetilde{S}_{n}\right)$ is the usual Serre condition $\left(S_{n}\right)$ for all $n$. If $M$ satisfies $\left(\widetilde{S}_{1}\right)$, then $M$ is a torsion-free $R$-module; in fact, $S^{-1} M$ is a torsion-free $S^{-1} R$-module for all multiplicatively closed subsets $S$ of $R$. If $R$ is a normal domain and $M$ satisfies $\left(\widetilde{S}_{2}\right)$, then $M$ is reflexive. The next result shows how a resolution of $M$ can be used to prove that $M$ satisfies $\left(\widetilde{S}_{n}\right)$ for some $n$. One may apply this result even when $I$ is the ideal (0).

Proposition 1.25. Let $M$ be a non-zero finitely generated $R$-module with $\operatorname{pd}_{R} M=$ $r$. Suppose that $I$ is a perfect grade $g$ ideal of $R$ with $I M=0$. For each integer $i$, with $1 \leq i \leq r$, let $F_{i}$ be the ideal of $R$ generated by

$$
\left\{x \in R \mid \operatorname{pd}_{R_{x}} M_{x}<i\right\} .
$$

If grade $F_{i} \geq i+n$ for all $i$ with $g+1 \leq i \leq r$, then the $(R / I)$-module $M$ satisfies the condition $\left(\widetilde{S}_{n}\right)$.

Note. If $P$ is a prime ideal of $R$, then $F_{i} \subseteq P$ if and only if $\operatorname{pd}_{R_{P}} M_{P} \geq i$.

Proof. Let $P$ be a prime ideal of $R$ which contains $I$. Observe, using (1.21), that

$$
g=\operatorname{grade} I \leq \operatorname{grade} I_{P} \leq \operatorname{pd}_{R_{P}}(R / I)_{P}
$$

When the Auslander-Buchsbaum equation is applied to the $R_{P}$-module $(R / I)_{P}$ we obtain:

$$
\operatorname{depth} R_{P}=\operatorname{pd}(R / I)_{P}+\operatorname{depth}(R / I)_{P} \geq g+\operatorname{depth}(R / I)_{P} .
$$

Let $i=\operatorname{pd} M_{P}$. If $i \leq g$, then the Auslander-Buchsbaum equation yields

$$
\operatorname{depth} M_{P}=\operatorname{depth} R_{P}-\operatorname{pd} M_{P} \geq\left(g+\operatorname{depth}(R / I)_{P}\right)-g=\operatorname{depth}(R / I)_{P} .
$$

If $g+1 \leq i \leq r$, then $F_{i} \subseteq P$; hence, grade $P \geq i+n$. Once again the AuslanderBuchsbaum equation yields

$$
\operatorname{depth} M_{P}=\operatorname{depth} R_{P}-\operatorname{pd} M_{P} \geq \operatorname{grade} P-i \geq(i+n)-i=n .
$$

A common application of the above result occurs when $\operatorname{pd}_{R} M \leq \operatorname{grade} I$. In this case there is no need to test the grade of any of the ideals $F_{i}$.

Corollary 1.26. If $I$ is a perfect ideal in $R$ and $M$ is a non-zero finitely generated $(R / I)$-module with $\operatorname{pd}_{R} M \leq$ grade $I$, then the $(R / I)$-module $M$ satisfies the condition $\left(\widetilde{S}_{n}\right)$ for all $n$; in particular, $M$ is torsion-free.

The $R$-module $M$ has rank $r$ if $M_{P}$ is a free $R_{P}$-module of rank $r$ for all $P \in \operatorname{Ass} R$. (Other, equivalent, definitions of rank may be found in section 16.A of [9].) We will often use the following 
Observation 1.27. If $\tau: M \rightarrow I$ is a surjection from a torsion-free $R$-module of rank one onto an $R$-ideal of positive grade, then $\tau$ is an isomorphism.

Occasionally, we will be forced to make an explicit calculation. We conclude this section by collecting the notation we use when dealing with sets of integers. If $s$ is a positive integer, then $[s]$ represents the set

$$
[s]=\{1,2, \ldots, s\} .
$$

Let $A=\left(a_{1}, \ldots, a_{i}\right)$ be an ordered $i$-tuple of integers. If the $a$ 's are distinct, then let

$$
\begin{aligned}
\sigma(A)= & \text { the sign of the permutation which rearranges } a_{1}, \ldots, a_{i} \text { into ascending } \\
& \text { order. }
\end{aligned}
$$

If there is a repeat among the $a$ 's, then let $\sigma(A)=0$. We say that $A$ is an index set of size $i$ if $a_{1}<a_{2}<\ldots<a_{i}$. Let $S$ be a fixed set of $s$ (distinct) integers. We write

$$
\sum_{\substack{A \cup B=S \\|A|=i}}
$$

to mean that the sum is taken over all $i$-element subsets $A$ of $S$, and the complement of $A$ in $S$ is denoted $B$. Furthermore, in the sum of (1.30) we think of $A$ and $B$ as index sets. Sometimes we will adjoin " $|B|=s-i$ " to the sum in (1.30). This addition has no effect on the meaning of (1.30). Let $x_{1}, \ldots, x_{s}$ be elements of a free module $E$. If $A=\left(a_{1}, \ldots, a_{i}\right)$ is an ordered $i$-tuple with each $a_{j}$ an element of $[s]$, then let $x_{A}$ be the element

$$
x_{A}=x_{a_{1}} \wedge \ldots \wedge x_{a_{i}} \text { of } \bigwedge^{i} E
$$

It is clear that the co-multiplication map $\Delta: \bigwedge^{s} E \rightarrow \bigwedge^{i} E \otimes \bigwedge^{s-i} E$ has been defined so that

$$
\Delta\left(x_{1} \wedge \ldots \wedge x_{s}\right)=\sum_{\substack{A \cup B=[s] \\|A|=i}} \sigma(A B) x_{A} \otimes x_{B}
$$




\section{Section 2. The definition of the family $\left\{\mathfrak{D}^{q}\right\}$.}

All of the modules, complexes, and ideals that we study in the rest of this paper are constructed from one almost alternating map.

Definition 2.1. Let $R$ be a commutative noetherian ring and let $f$ and $g$ be integers with $f \geq 0$ and $g \geq 1$. An almost alternating map $\rho$ of shape $(g, f)$ over $R$ is a map

$$
\rho=[\xi \psi]: G^{*} \oplus F \rightarrow G,
$$

where $F$ and $G$ are free $R$-modules of rank $f$ and $g$ respectively, $\xi$ is an alternating map, and $\psi$ is an arbitrary map.

Remarks 2.2.

(a) The definition of $\rho$ means that $\rho \mid G^{*}=\xi$ and $\rho \mid F=\psi$.

(b) See (1.17) for the definition of "alternating map".

(c) Whenever we choose bases $\gamma_{1}, \ldots, \gamma_{g}$ and $\phi_{1}, \ldots, \phi_{f}$ for $G$ and $F$ (and the corresponding dual basis $\gamma_{1}^{*}, \ldots, \gamma_{g}^{*}$ for $G^{*}$ and $\phi_{1}^{*}, \ldots, \phi_{f}^{*}$ for $F^{*}$ ), then we let $X$ be the matrix for $\xi$ and $Y$ be the matrix for $\psi$.

(d) It is reasonable to refer to the matrix $[X Y]$ as an "almost alternating matrix"; indeed, it is as close to an alternating matrix as a not necessarily square matrix can be.

(e) We say that the almost alternating map $\rho$ is generic if $R$ is a polynomial $\operatorname{ring} R_{0}\left[\left\{x_{i j}, y_{i j}\right\}\right], \xi$ is the generic alternating map given by $X=\left(x_{i j}\right)$ and $\psi$ is the generic map given by $Y=\left(y_{i j}\right)$. Each of the indeterminates $x_{i j}$ and $y_{i j}$ is given degree one.

Throughout the rest of the paper we use the following

Data 2.3. Let $\rho=[\xi \psi]: G^{*} \oplus F \rightarrow G$ be an almost alternating map of shape $(g, f)$ over the commutative noetherian ring $R$. Let $E$ be the module $G \oplus F^{*}$, let $e$ be the integer $f+g$, let $\pi$ denote the projection map

$$
\pi: E=G \oplus F^{*} \rightarrow G,
$$

and let $\theta$ be the alternating map

$$
\theta=\left[\begin{array}{cc}
\xi & \psi \\
-\psi^{*} & 0
\end{array}\right]: E^{*}=G^{*} \oplus F \rightarrow E=G \oplus F^{*} .
$$

Remark 2.4. The data of (2.3) is defined so that the diagram

$$
\begin{gathered}
E^{*}=G^{*} \oplus \underset{\theta^{\prime}}{\rightarrow} E=G \oplus F^{*} \\
\rho \searrow \swarrow \pi \\
G
\end{gathered}
$$

commutes. For each even integer $2 p$, the $p^{\text {th }}$ divided power of $\theta$ is the map

$$
\theta^{(p)}: \bigwedge^{2 p} E^{*} \rightarrow R
$$

as defined above Observation 1.19. 
In $(2.15)$ we define $R$-modules $Q_{i}=Q_{i}(\rho), K_{i j}=K_{i j}(\rho)$, and $L_{i j}=L_{i j}(\rho)$ with

$$
Q_{i} \subseteq \grave{\Lambda} F, \quad S_{i} G \otimes \bigwedge^{j} E^{*} \rightarrow K_{i j}, \quad \text { and } \quad L_{i j} \subseteq D_{i} G^{*} \otimes \bigwedge^{j} E^{*}
$$

and $R$-module homomorphisms

$$
\begin{aligned}
d: L_{a b} \rightarrow L_{a-1 b-1}, \quad & d: L_{0 g+q+2} \rightarrow Q_{q}, \quad d: Q_{q} \rightarrow K_{0 q} \\
\text { and } \quad d: K_{a b} \rightarrow K_{a+1 b-1} . &
\end{aligned}
$$

For each integer $q$, we define $\left(\mathfrak{D}^{q}, d\right)=\left(\mathfrak{D}^{q}(\rho), d\right)$ to be:

$$
\ldots \rightarrow L_{1 g+q+3} \rightarrow L_{0 g+q+2} \rightarrow Q_{q} \rightarrow K_{0 q} \rightarrow K_{1 q-1} \rightarrow \ldots
$$

with $Q_{q}$ in position $q+1$. The rest of this section and all of the next section are devoted to proving that each $\left(\mathfrak{D}^{q}, d\right)$ is a complex of free $R$-modules. (Some qualitative information about the family $\left\{\mathfrak{D}^{q}\right\}$, including a picture, may be found in section four.) Before giving our official definition of the modules and maps in (2.5), we outline our procedure. Let

$$
(\mathbb{K}, \partial)=\left(S \bullet G \otimes \grave{\bigwedge} E^{*}, \partial\right)
$$

be the Koszul algebra associated to $\rho: E^{*} \rightarrow G$. (See (1.8), if necessary.) In Proposition 2.17 we show that the part of $\left(\mathfrak{D}^{q}, d\right)$ "below the diagonal":

$$
K_{0 q} \rightarrow K_{1 q-1} \rightarrow \ldots \rightarrow K_{q-11} \rightarrow K_{q 0} \rightarrow 0,
$$

is a complex by identifying a differential ideal $\mathcal{J}$ of $\mathbb{K}$ such that (2.7) is a graded strand of the differential bigraded algebra $K_{\bullet} \bullet=(\mathbb{K} / \mathcal{J}, \partial)$. In Proposition 2.21 we prove that the composition

$$
Q_{q} \rightarrow K_{0 q} \rightarrow K_{1 q-1}
$$

is zero; hence $\left(\mathfrak{D}_{\text {right }}^{q}, d\right)$ :

$$
Q_{q} \rightarrow K_{0 q} \rightarrow K_{1 q-1} \rightarrow \ldots \rightarrow K_{q-11} \rightarrow K_{q 0} \rightarrow 0
$$

is a complex. Let $\left(\mathfrak{D}_{\text {left }}^{q}, d\right)$ represent

$$
0 \rightarrow L_{e-g-q-2 e} \rightarrow L_{e-g-q-3 e-1} \rightarrow \ldots \rightarrow L_{1 g+q+3} \rightarrow L_{0 g+q+2} \rightarrow Q_{q}
$$

We prove that the maps of $\mathfrak{D}_{\text {left }}^{q}$ are well defined and that $\mathfrak{D}_{\text {left }}^{q}$ is a complex by showing, in Proposition 2.23, that there is an isomorphism of complexes between $\mathfrak{D}_{\text {left }}^{q}$ and $\left(\mathfrak{D}_{\text {right }}^{f-2-q}\right)^{*}[-f]$. In section 3 we prove that the composition

$$
L_{0 g+q+2} \rightarrow Q_{q} \rightarrow K_{0 q}
$$


is zero, from which it follows that $\mathfrak{D}^{q}$ is a complex. We prove that the modules in $\mathfrak{D}^{q}$ are free and relate these modules to the modules $L_{p}^{q}$ of Buchsbaum and Eisenbud in Proposition 2.41.

We must set up a small amount of preliminary notation before we can define the constituent pieces of $\mathfrak{D}^{q}$. There is a natural decomposition

$$
\bigwedge^{j} E^{*} \cong \bigoplus_{a+b=j} \bigwedge^{a} G^{*} \otimes \bigwedge^{b} F
$$

consequently, if $a+b=j$, then there are natural maps

$$
\bigwedge^{a} G^{*} \otimes \bigwedge^{b} F \stackrel{\text { incl }}{\longrightarrow} \bigwedge^{j} E^{*}, \quad \text { and } \quad \bigwedge^{j} E^{*} \stackrel{\text { proj }}{\longrightarrow} \bigwedge^{a} G^{*} \otimes \bigwedge^{b} F .
$$

We consider two elements

$$
\eta \in S_{1} G \otimes \bigwedge^{1} E^{*} \subseteq \mathbb{K} \quad \text { and } \quad \lambda \in S_{0} G \otimes \bigwedge^{g} E^{*} \subseteq \mathbb{K}
$$

of the algebra $\mathbb{K}=\left(S \bullet G \otimes \bigwedge^{\bullet} E^{*}, \partial\right)$, which is defined in (2.6). There is a natural isomorphism

$$
G \otimes E^{*} \rightarrow \operatorname{Hom}(E, G) .
$$

Let $\eta$ be the element of $G \otimes E^{*}$ which corresponds to the projection $\pi: E=G \oplus F^{*} \rightarrow$ $G$ under the above isomorphism. Let $\lambda$ be the element of

$$
\bigwedge^{g} G^{*}=\bigwedge^{g} G^{*} \otimes \bigwedge^{0} F \stackrel{\text { incl }}{\longrightarrow} \bigwedge^{g} E^{*}=S_{0} G \otimes \bigwedge^{g} E^{*}
$$

with $[\lambda]=1$, where [ ]: $\bigwedge^{g} G^{*} \rightarrow R$ is the orientation isomorphism of (1.4). Finally, the inclusion $G^{*} \hookrightarrow E^{*}=G^{*} \oplus F$ induces a map

$$
\nu: D_{i} G^{*} \otimes \bigwedge^{j} E^{*} \rightarrow D_{i-1} G^{*} \otimes \bigwedge^{j+1} E^{*},
$$

where $\nu$ is the composition:

$$
\begin{gathered}
D_{i} G^{*} \otimes \bigwedge^{j} E^{*} \stackrel{\Delta \otimes 1}{\longrightarrow} D_{i-1} G^{*} \otimes G^{*} \otimes \bigwedge^{j} E^{*} \stackrel{1 \otimes \operatorname{incl} \otimes 1}{\longrightarrow} \\
D_{i-1} G^{*} \otimes E^{*} \otimes \bigwedge^{j} E^{*} \stackrel{1 \otimes \mu}{\longrightarrow} D_{i-1} G^{*} \otimes \bigwedge^{j+1} E^{*} .
\end{gathered}
$$

Definition 2.15. Let $i, j$, and $q$ be integers.

(a) The module $K_{i j}$ is defined to be cokernel:

$$
\begin{aligned}
& S_{i-1} G \otimes \bigwedge^{j-1} E^{*} \stackrel{\eta}{\rightarrow} S_{i} G \otimes \bigwedge^{j} E^{*} \rightarrow K_{i j} \rightarrow 0 \quad \text { if } \quad i>0, \quad \text { and } \\
& S_{0} G \otimes \bigwedge^{j-g} E^{*} \stackrel{\lambda}{\rightarrow} S_{0} G \otimes \bigwedge^{j} E^{*} \rightarrow K_{0 j} \rightarrow 0 .
\end{aligned}
$$

The first map in each sequence is multiplication from the left by an element of $S_{\bullet} G \otimes \Lambda^{\bullet} E^{*}$. 
(b) The module $L_{i j}$ is defined to be the kernel:

$$
\begin{aligned}
& 0 \rightarrow L_{i j} \rightarrow D_{i} G^{*} \otimes \bigwedge^{j} E^{*} \stackrel{\nu}{\rightarrow} D_{i-1} G^{*} \otimes \bigwedge^{j+1} E^{*} \quad \text { if } \quad i>0, \quad \text { and } \\
& 0 \rightarrow L_{0 j} \rightarrow D_{0} G^{*} \otimes \bigwedge^{j} E^{*} \stackrel{\text { proj }}{\longrightarrow} D_{0} G^{*} \otimes \bigwedge^{0} G^{*} \otimes \bigwedge^{j} F
\end{aligned}
$$

(c) The module $Q_{q}$ is equal to $\oplus_{r} \Lambda^{r} F$, where the sum is taken over all integers $r$ with

$$
g+r-q \text { even } \quad \text { and } \quad q-g+2 \leq r \leq q+g .
$$

(d) The map $d: K_{a b} \rightarrow K_{a+1 b-1}$ is induced by the Koszul complex map

$$
\partial: S_{a} G \otimes \bigwedge^{b} E^{*} \rightarrow S_{a+1} G \otimes \bigwedge^{b-1} E^{*}
$$

of (1.9) associated to $\rho: E^{*} \rightarrow G$.

(e) For $a>0$, the map $d: L_{a b} \rightarrow L_{a-1 b-1}$ is induced by the Eagon-Northcott $\operatorname{map} \delta: D_{a} G^{*} \otimes \bigwedge^{b} E^{*} \rightarrow D_{a-1} G^{*} \otimes \bigwedge^{b-1} E^{*}$ of (1.12) associated to $\rho: E^{*} \rightarrow G$.

(f) The map $d: Q_{q} \rightarrow K_{0 q}$ is defined by letting the restriction of $d$ to $\wedge^{r} F$ be the composition:

$$
\begin{gathered}
\bigwedge_{r} F \stackrel{\lambda}{\longrightarrow} \bigwedge^{g} G^{*} \otimes \bigwedge^{r} F \stackrel{\text { incl }}{\longrightarrow} \bigwedge^{g+r} E^{*} \stackrel{\Delta}{\longrightarrow} \bigwedge^{2 p} E^{*} \otimes \bigwedge^{q} E^{*} \\
\stackrel{\theta^{(p)} \otimes \text { id }}{\longrightarrow} R \otimes \bigwedge^{q} E^{*}=S_{0} G \otimes \bigwedge^{q} E^{*} \stackrel{\text { nat }}{\longrightarrow} K_{0 q},
\end{gathered}
$$

where $p$ is the integer $(g+r-q) / 2$. The maps $\lambda$ and nat are described in (a).

(g) Fix integers $a, b$, and $r$ so that $a+b=g+q+2, a \neq 0$, and $r$ satisfies the restrictions of (2.16). The component

$$
\bigwedge^{a} G^{*} \otimes \bigwedge^{b} F \stackrel{\text { incl }}{\longrightarrow} L_{0} g+q+2 \stackrel{d}{\rightarrow} Q_{q} \stackrel{\text { proj }}{\longrightarrow} \bigwedge^{r} F
$$

of $d$ is defined to be the composition

$$
\begin{gathered}
\bigwedge^{a} G^{*} \otimes \bigwedge^{b} F \stackrel{1 \otimes \Delta}{\longrightarrow} \bigwedge^{a} G^{*} \otimes \bigwedge^{b-r} F \otimes \bigwedge^{r} F \stackrel{\text { incl }}{\longrightarrow} \bigwedge^{2 p} E^{*} \otimes \bigwedge^{r} F \\
\stackrel{\theta^{(p)} \otimes 1}{\longrightarrow} R \otimes \bigwedge^{r} F=\bigwedge^{r} \stackrel{(-1)^{p}}{\longrightarrow} \bigwedge^{r} F
\end{gathered}
$$

for $p=(a+b-r) / 2$.

Proposition 2.17. The modules and maps of (2.7) form a complex.

Proof. Let $\mathcal{J}$ be the ideal of $\mathbb{K}$ generated by $\eta$ and $\lambda$ of (2.13). Parts (a) and (b) of Observation 2.18 show that $\mathcal{J}$ is a bigraded $D G$-algebra ideal of $\mathbb{K}$; and consequently, $K_{\bullet} \bullet=\mathbb{K} / \mathcal{J}$ is a differential bigraded algebra. Part (c) of Observation 2.18 shows that line $(2.7)$ is a graded strand of $K_{\bullet}$ •. 


\section{Observation 2.18.}
(a) $\partial(\eta)=0$
(b) $\partial(\lambda) \in \eta \mathbb{K}$
(c) $\lambda\left(S_{+} G \otimes \bigwedge^{\bullet} E^{*}\right) \subseteq \eta \mathbb{K}$

Proof. Pick bases for $G$ and $G^{*}$ as in Remark 2.2 (c). It is clear that

$$
\eta=\sum_{1}^{g} \gamma_{i} \otimes \gamma_{i}^{*} \in S_{1} G \otimes \bigwedge^{1} E^{*} \quad \text { and } \quad \lambda=\gamma_{1}^{*} \wedge \ldots \wedge \gamma_{g}^{*} \in S_{0} G \otimes \bigwedge^{g} E^{*}
$$

If $x$ is any element of $G$, then when we write $x$ in terms of the basis for $G$ we obtain

$$
x=\sum_{1}^{g}\left\langle\gamma_{j}^{*}, x\right\rangle \gamma_{j}
$$

(a) Recall that $\rho \mid G^{*}$ is the map $\xi$. It follows, from (2.20), that

$$
\partial(\eta)=\sum_{i=1}^{g} \gamma_{i} \xi\left(\gamma_{i}^{*}\right)=\sum_{i, j=1}^{g}\left\langle\gamma_{j}^{*}, \xi\left(\gamma_{i}^{*}\right)\right\rangle \gamma_{i} \gamma_{j} \in S_{2} G .
$$

We conclude that $\partial \eta=0$ because $\xi$ is an alternating map.

(b) Let $y$ be the element

$$
\sum_{a<b}(-1)^{a+b}\left\langle\gamma_{a}^{*}, \xi\left(\gamma_{b}^{*}\right)\right\rangle \gamma_{1}^{*} \wedge \ldots \wedge \widehat{\gamma_{a}^{*}} \wedge \ldots \wedge \widehat{\gamma_{b}^{*}} \wedge \ldots \wedge \gamma_{g}^{*}
$$

of $S_{0} G \otimes \bigwedge^{g-2} E^{*} \subseteq \mathbb{K}$. Observe that

$$
\eta y=\sum_{b=1}^{g}(-1)^{b+1}\left[\sum_{j=1}^{g}\left\langle\gamma_{j}^{*}, \xi\left(\gamma_{b}^{*}\right)\right\rangle \gamma_{j}\right] \otimes \gamma_{1}^{*} \wedge \ldots \wedge \widehat{\gamma_{b}^{*}} \wedge \ldots \wedge \gamma_{g}^{*} .
$$

Thus, (2.20) shows that

$$
\eta y=\sum_{b=1}^{g}(-1)^{b+1} \xi\left(\gamma_{b}^{*}\right) \otimes \gamma_{1}^{*} \wedge \ldots \wedge \widehat{\gamma_{b}^{*}} \wedge \ldots \wedge \gamma_{g}^{*}=\partial(\lambda) .
$$

(c) The final assertion is obvious because, for example,

$$
\gamma_{1} \lambda=\eta\left(\gamma_{2}^{*} \wedge \ldots \wedge \gamma_{g}^{*}\right)
$$


Proposition 2.21. The maps and modules $\left(\mathfrak{D}_{\text {right }}^{q}, d\right)$ in $(2.9)$ form a complex.

Proof. It suffices to prove that the composition of (2.8) is zero. Fix an integer $r$ which satisfies the restrictions of (2.16). Let $p$ be the integer

$$
p=(g+r-q) / 2 \text {. }
$$

Observe that the constraints on $r$ are equivalent to

$$
1 \leq p \leq g
$$

We establish the result by proving that the diagram

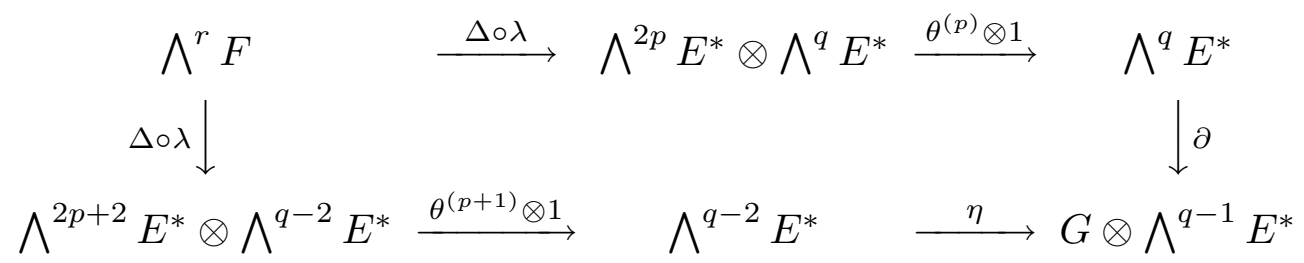

commutes. (Indeed, the composition of (2.8), when restricted to the summand $\bigwedge^{r} F$ of $Q_{q}$, is

$$
\bigwedge_{r} F \rightarrow G \otimes \bigwedge^{q-1} E^{*} \rightarrow \frac{G \otimes \bigwedge^{q-1} E^{*}}{\eta\left(\bigwedge^{q-2} E^{*}\right)}=K_{1 q-1}
$$

where the first map is the "clockwise map" in the above diagram and the second map is the natural quotient map.)

Let $x_{g+1}, \ldots, x_{g+r}$ be arbitrary elements of $F$, let $s$ be the integer $g+r$, let $X$ be the element $x_{g+1} \wedge \ldots \wedge x_{s}$ of $\wedge^{r} F$, and let $\operatorname{im} X$ be the element

$$
\operatorname{im} X=\partial \circ\left(\theta^{(p)} \otimes 1\right) \circ \Delta(\lambda X)
$$

of $G \otimes \bigwedge^{q-1} E^{*}$. For $1 \leq i \leq g$, let $x_{i}$ be the element $\gamma_{i}^{*}$ of $G^{*} \subseteq E^{*}$. We know, from (2.19), that $\lambda X=x_{1} \wedge \ldots \wedge x_{s}$ in $\wedge^{s} E^{*}$. According to (1.32), $\operatorname{im} X$ is equal to

$$
\partial \circ\left(\theta^{(p)} \otimes 1\right) \circ \Delta\left(x_{1} \wedge \ldots \wedge x_{s}\right)=\sum_{\substack{A \cup B=[s] \\|A|=2 p \\|B|=q}} \sigma(A B) \theta^{(p)}\left(x_{A}\right) \partial\left(x_{B}\right) .
$$

If we continue to use the notation of (1.32), then we see that

$$
\partial\left(x_{B}\right)=\sum_{\substack{\{i\} \cup C=B \\|C|=q-1}} \sigma(i C) \rho\left(x_{i}\right) x_{C}
$$

Recall, from Remark 2.4, that $\rho=\pi \theta$. Consequently, when $\rho\left(x_{i}\right)$ is expressed in terms of the basis for $G$, as in (2.20), we obtain

$$
\rho\left(x_{i}\right)=\sum_{j=1}^{g}\left\langle\gamma_{j}^{*}, \theta\left(x_{i}\right)\right\rangle \gamma_{j}=-\sum_{j=1}^{g}\left\langle\theta\left(\gamma_{j}^{*}\right), x_{i}\right\rangle \gamma_{j}
$$


We change the order of summation in order to obtain that $\operatorname{im} X$ is equal to

$$
-\sum_{j=1}^{g} \sum_{\substack{D \cup C=[s] \\|D|=2 p+1 \\|C|=q-1}} \sigma(D C)\left[\sum_{\substack{\{i\} \cup A=D \\|A|=2 p}} \sigma(i A)\left\langle\theta \gamma_{j}^{*}, x_{i}\right\rangle \theta^{(p)}\left(x_{A}\right)\right] \gamma_{j} \otimes x_{C}
$$

(Notice that $\sigma(A B) \sigma(i C)=\sigma(A i C)=\sigma(i A C)=\sigma(i A) \sigma(D C)$, where the middle equality holds because $A$ has even cardinality.) The pfaffian expansion formula of (1.20) shows that the expression inside the brackets is equal to $\theta^{(p+1)}\left(\gamma_{j}^{*} \wedge x_{D}\right)$; consequently $\operatorname{im} X$ is equal to

$$
-\sum_{j=1}^{g} \sum_{\substack{D \cup C=[s] \\|D|=2 p+1 \\|C|=q-1}} \sigma(D C) \theta^{(p+1)}\left(\gamma_{j}^{*} \wedge x_{D}\right) \gamma_{j} \otimes x_{C}
$$

The number $j$ must appear in either $D$ or $C$. If $j$ is in $D$, then the corresponding term is zero because $x_{j}=\gamma_{j}^{*}$. So we may assume $j \in C$; in other words, $C=\{j\} \cup E$ for some set $E$. After a slight amount of further index shuffling we see that $\operatorname{im} X$ equals

$$
\begin{aligned}
& \sum_{j=1}^{g} \sum_{\substack{0 \cup E=[s] \backslash\{j\} \\
|D|=2 p+1 \\
|E|=q-2}} \sigma(j D E) \theta^{(p+1)}\left(\gamma_{j}^{*} \wedge x_{D}\right) \gamma_{j} \otimes \gamma_{j}^{*} \wedge x_{E} \\
& \quad=\sum_{j=1}^{g} \sum_{\substack{G \cup E=[s] \\
|G|=2 p+2 \\
|E|=q-2}} \sigma(G E) \theta^{(p+1)}\left(x_{G}\right) \gamma_{j} \otimes \gamma_{j}^{*} \wedge x_{E} \\
& =\sum_{j=1}^{g}\left(\gamma_{j} \otimes \gamma_{j}^{*}\right)\left(\theta^{(p+1)} \otimes 1\right) \Delta(\lambda X)=\eta\left(\theta^{(p+1)} \otimes 1\right) \Delta(\lambda X) .
\end{aligned}
$$

Proposition 2.23. The sequence of maps and modules $\left(\mathfrak{D}_{\mathrm{left}}^{q}, d\right)$ of $(2.10)$ is a complex. Furthermore, the complexes $\mathfrak{D}_{\text {left }}^{q}$ and $\left(\mathfrak{D}_{\text {right }}^{f-2-q}\right)^{*}[-f]$ are isomorphic.

Proof. In light of Proposition 2.21, it suffices to prove the second assertion. We begin by proving that

$$
L_{i j} \cong\left(K_{i e-j}\right)^{*}
$$

for all integers $i$ and $j$. We first take $i=0$. The three orientation isomorphisms of $(1.4)$ :

$$
\left[\mathrm{]}: \bigwedge^{e} E^{*} \rightarrow R, \quad\left[\mathrm { ] } : \bigwedge ^ { g } G ^ { * } \rightarrow R , \quad \text { and } \quad \left[\mathrm{]}: \bigwedge^{f} F \rightarrow R\right.\right.\right.
$$


are compatible in the sense that the diagram

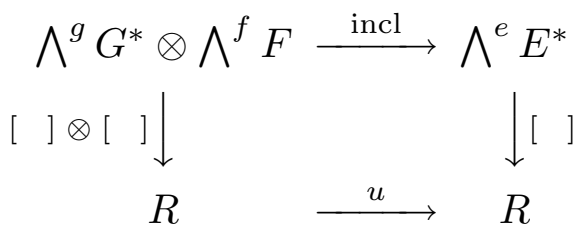

commutes for some unit $u$ of $R$. We may assume, without loss of generality, that $u=1$. It is immediate that the pairing of (1.5) induces a commutative square:

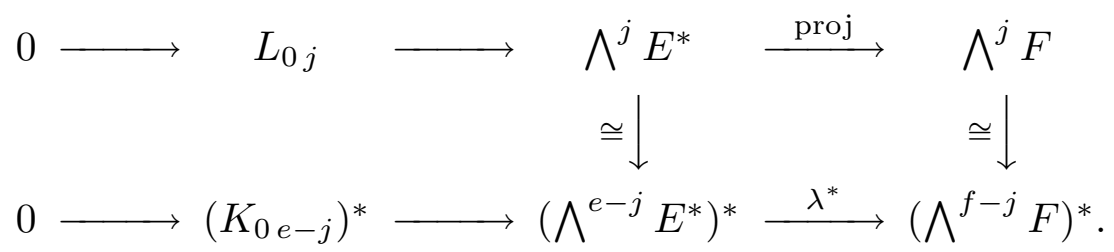

Indeed, if $x \in \bigwedge^{j} E^{*}$ and $y \in \bigwedge^{f-j} F$, then

$$
[(\operatorname{proj} x) \wedge y]=[\lambda \wedge x \wedge y]
$$

We conclude that (2.24) holds for $i=0$.

We next show that (2.24) holds for $i>0$. Let

$$
\langle\langle, \quad\rangle\rangle:\left(D_{i} G^{*} \otimes \bigwedge^{j} E^{*}\right) \otimes\left(S_{i} G \otimes \bigwedge^{e-j} E^{*}\right) \rightarrow R
$$

be the perfect pairing of (1.14), and let

$$
\nu: D_{i} G^{*} \otimes \bigwedge^{j} E^{*} \rightarrow D_{i-1} G^{*} \otimes \bigwedge^{j+1} E^{*}
$$

be the map of (2.14). If $x \in D_{i} G^{*}, y \in \bigwedge^{j} E^{*}, z \in S_{i-1} G$, and $w \in \bigwedge^{e-j-1} E^{*}$, then it is easy to see that

$$
\begin{aligned}
& \langle\langle x \otimes y, \eta(z \otimes w)\rangle\rangle=\sum_{i=1}^{g}\left\langle\left\langle x, \gamma_{i} z\right\rangle\right\rangle\left[y \wedge\left(\gamma_{i}^{*} \wedge w\right)\right], \quad \text { and } \\
& \langle\langle\nu(x \otimes y), z \otimes w\rangle\rangle=\sum_{i=1}^{g}\left\langle\left\langle x, \gamma_{i} x\right\rangle\right\rangle\left[\left(\gamma_{i}^{*} \wedge y\right) \wedge w\right]
\end{aligned}
$$

where $\left\langle\left\langle x, \gamma_{i} z\right\rangle\right\rangle$ is the perfect pairing of (1.3) and [ ] is the orientation isomorphism of (1.4). Since these elements of $R$ differ only by a factor of $(-1)^{j}$ we conclude that $\langle\langle\rangle$,$\rangle induces a commutative square:$

$$
\begin{aligned}
& 0 \longrightarrow L_{i j} \longrightarrow D_{i} G^{*} \otimes \bigwedge^{j} E^{*} \stackrel{\nu}{\longrightarrow} D_{i-1} G^{*} \otimes \bigwedge^{j+1} E^{*} \\
& \cong \downarrow \\
& 0 \longrightarrow\left(K_{i e-j}\right)^{*} \longrightarrow\left(S_{i} G \otimes \bigwedge^{e-j} E^{*}\right)^{*} \stackrel{\eta^{*}}{\longrightarrow}\left(S_{i-1} G \otimes \bigwedge^{e-j-1} E^{*}\right)^{*} \text {; }
\end{aligned}
$$


and consequently, (2.24) holds for $i>0$.

Fix $i>0$. Consider the diagram:

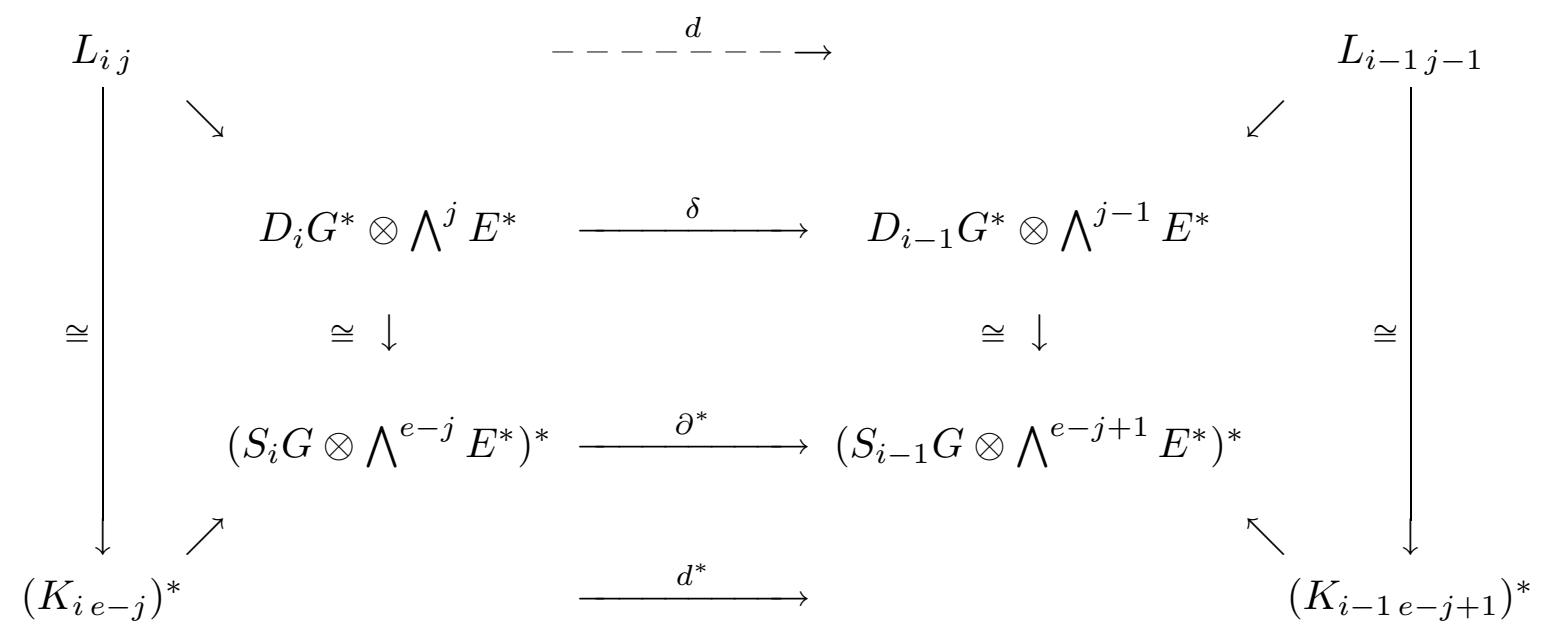

The side quadrilaterals commute because of (2.27) (and (2.26) if $i=1$ ). The bottom quadrilateral is the dual of Definition 2.15 (d) (by way of Proposition 2.17). The middle square is the dual of (1.16). We conclude that the image of the map

$$
d: L_{i j} \rightarrow D_{i-1} G^{*} \otimes \bigwedge^{j-i} E^{*}
$$

of Definition 2.15 (e) is contained in $L_{i-1 j-1}$, as desired. We also conclude that the complexes

$$
\begin{aligned}
& 0 \rightarrow L_{e-a e} \rightarrow \ldots \rightarrow \quad L_{1 a+1} \rightarrow L_{0 a}, \quad \text { and } \\
& 0 \rightarrow\left(K_{e-a 0}\right)^{*} \rightarrow \ldots \rightarrow\left(K_{1 e-a-1}\right)^{*} \rightarrow\left(K_{0 e-a}\right)^{*}
\end{aligned}
$$

are isomorphic for all integers $a$.

The proof will be completed once we define an isomorphism

$$
\alpha_{q}: Q_{q} \rightarrow Q_{f-2-q}^{*}
$$

which makes the square

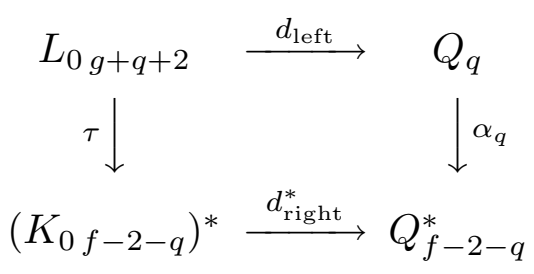

commute, where the maps $d_{\text {right }}$ and $d_{\text {left }}$ are found in Definition $2.15(\mathrm{f})$ and $(\mathrm{g})$, respectively; and the map $\tau$ is described in (2.26). In particular, $\tau$ is induced by the perfect pairing

$$
\bigwedge^{g+q+2} E^{*} \otimes \bigwedge^{f-2-q} E^{*} \rightarrow R
$$


of (1.5). (Remember that $f+g=e$.) In this part of the calculation it is useful for us to be a little more explicit; so we define

$$
\tau: \bigwedge^{g+q+2} E^{*} \rightarrow\left(\bigwedge^{f-2-q} E^{*}\right)^{*}
$$

by $\tau(x)=[-\wedge x] \in\left(\bigwedge^{f-2-q} E^{*}\right)^{*}$ for all $x \in \bigwedge^{g+q+2} E^{*}$.

The $Q$ modules are defined in $(2.15 \mathrm{c})$ to be

$$
Q_{q}=\bigoplus_{f-r} \bigwedge^{f-r} F \quad \text { and } \quad Q_{f-2-q}=\bigoplus_{r} \bigwedge^{r} F,
$$

where the integers $f-r$ satisfy

$$
g+(f-r)-q \text { even and } \quad q-g+2 \leq(f-r) \leq q+g
$$

for $Q_{q}$; and the integers $r$ satisfy

$$
g+r-(f-2-q) \quad \text { even and } \quad(f-2-q)-g+2 \leq r \leq(f-2-q)+g
$$

for $Q_{f-2-q}$. It is clear that (2.30) and (2.31) put exactly the same restrictions on $r$. We define the isomorphism $\alpha_{q}$ of (2.28) by

$$
\alpha_{q}(x)=(-1)^{(g+r+q-f+2) / 2}[-\wedge x] \in\left(\bigwedge^{r} F\right)^{*}
$$

for each $x \in \bigwedge^{f-r} F$ and for each $r$ which satisfies the restrictions of (2.31). Observe, for future reference, that

$$
\alpha_{f-2-q}=u \alpha_{q}^{*}
$$

for some unit $u$ of $R$. Indeed, if $r$ is a fixed integer which satisfies (2.31) and $y$ is an element of $\bigwedge^{r} F \subseteq Q_{f-2-q}$, then

$$
\begin{aligned}
\alpha_{q}^{*}(y) & =(-1)^{(g+r+q-f+2) / 2}[y \wedge-] \in\left(\bigwedge^{f-r} F\right)^{*}, \quad \text { and } \\
\alpha_{f-2-q}(y) & =(-1)^{(g+f-r-q) / 2}[-\wedge y] \in\left(\bigwedge^{f-r} F\right)^{*} .
\end{aligned}
$$

It is clear that $[y \wedge x]=(-1)^{r(f-r)}[x \wedge y]$. On the other hand $(-1)^{r(f-r)+g+1}$ depends only on $f, g$, and $q$.

Now that $\alpha_{q}$ has been defined, we return to the left side of (2.29). Recall from Definition 2.15 (a) and (b) that

$$
\begin{aligned}
K_{0 f-2-q} & =\frac{\bigwedge^{f-2-q} E^{*}}{\bigwedge^{g} G^{*} \otimes \bigwedge^{f-2-q-g} F}=\sum_{\substack{a+b=f-q-2 \\
a \neq g}} \bigwedge^{a} G^{*} \otimes \bigwedge^{b} F, \quad \text { and } \\
L_{0 g+2+q} & =\frac{\bigwedge^{g+2+q} E^{*}}{\bigwedge^{0} G^{*} \otimes \bigwedge^{g+q+2} F}=\sum_{\substack{(g-a)+(f-b)=g+2+q \\
g-a \neq 0}} \bigwedge^{g-a} G^{*} \otimes \bigwedge^{f-b} F \\
& =\sum_{\substack{a+b=f-q-2 \\
a \neq g}} \bigwedge^{g-a} G^{*} \otimes \bigwedge^{f-b} F .
\end{aligned}
$$


Fix $a, b$, and $r$ with

$(2.33)$

$a+b=f-2-q, \quad(g-a)+(r-b)$ even, $\quad$ and $\quad a+b+2-g \leq r \leq g+a+b$.

We prove that (2.29) commutes by proving that

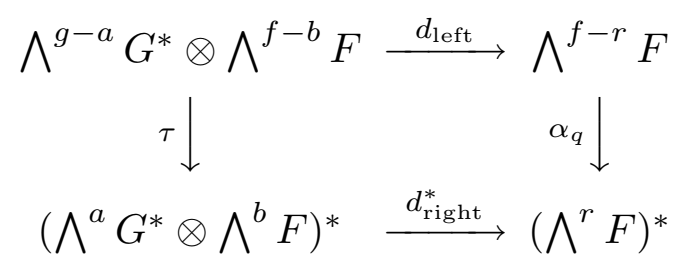

commutes. In other words, we show that

$$
\left[d_{\text {right }} z \wedge x \wedge y\right]=(-1)^{(g+r+q+2-f) / 2}\left[z \wedge d_{\text {left }}(x \otimes y)\right]
$$

for all $x \in \bigwedge^{g-a} G^{*}, y \in \bigwedge^{f-b} F$, and $z \in \bigwedge^{r} F$. (The orientation on the left is [ ] : $\bigwedge^{e} E^{*} \rightarrow R$; the orientation on the right is [ ]: $\bigwedge^{f} F \rightarrow R$.) Let $p$ be the integer defined by $2 p=(g-a)+(r-b)$. The restriction on $r$ in (2.33) is equivalent to $1 \leq p \leq g$ and (2.35) may be rewritten as

$$
\left[d_{\text {right }} z \wedge x \wedge y\right]=(-1)^{p}\left[z \wedge d_{\text {left }}(x \otimes y)\right] .
$$

The component $d_{\text {left }}: \bigwedge^{g-a} G^{*} \otimes \bigwedge^{f-b} F \rightarrow \bigwedge^{f-r} F \quad$ of $d: L_{0 g+q+2} \rightarrow Q_{q}$ which is used in (2.34) is the composition:

$$
\begin{gathered}
\bigwedge^{g-a} G^{*} \otimes \bigwedge^{f-b} F \stackrel{1 \otimes \Delta}{\longrightarrow} \bigwedge^{g-a} G^{*} \otimes \bigwedge^{r-b} F \otimes \bigwedge^{f-r} F \\
\stackrel{\text { incl } \otimes 1}{\longrightarrow} \bigwedge^{2 p} E^{*} \otimes \bigwedge^{f-r} F \stackrel{\theta^{(p)} \otimes 1}{\longrightarrow} \bigwedge^{f-r} F \stackrel{(-1)^{p}}{\longrightarrow} \bigwedge^{f-r} F
\end{gathered}
$$

The component $d_{\text {right }}: \bigwedge^{r} F \rightarrow \bigwedge^{a} G^{*} \otimes \bigwedge^{b} F$ of $d: Q_{f-2-q} \rightarrow K_{0 f-2-q}$ which is used in (2.34) is the composition.

$$
\begin{aligned}
\bigwedge_{r} F \stackrel{\lambda}{\longrightarrow} \bigwedge^{g} G^{*} \otimes & \bigwedge^{r} F \hookrightarrow \bigwedge^{g+r} E^{*} \stackrel{\Delta}{\longrightarrow} \bigwedge^{2 p} E^{*} \otimes \bigwedge^{a+b} E^{*} \\
& \stackrel{\theta^{(p)} \otimes 1}{\longrightarrow} \bigwedge^{a+b} E^{*} \stackrel{\operatorname{proj}}{\longrightarrow} \bigwedge^{a} G^{*} \otimes \bigwedge^{b} F .
\end{aligned}
$$

Choose bases for $F$ and $G$ as in Remark 2.2 (c). We may as well assume that

$$
x=\gamma_{i_{1}}^{*} \wedge \ldots \wedge \gamma_{i_{g-a}}^{*}, \quad y=\phi_{j_{1}} \wedge \ldots \wedge \phi_{j_{f-b}}, \quad \text { and } \quad z=\phi_{k_{1}} \wedge \ldots \wedge \phi_{k_{r}} .
$$

Observe that both sides of (2.36) are zero unless

$$
\left\{j_{1}, \ldots, j_{f-b}, k_{1}, \ldots, k_{r}\right\}=[f] .
$$


(See (1.28).) In this case, the intersection

$$
\left\{j_{1}, \ldots, j_{f-b}\right\} \cap\left\{k_{1}, \ldots, k_{r}\right\}
$$

has cardinality $r-b$.

Let $x_{2}$ be the basis element in $\bigwedge^{a} G^{*}$ with $\left[x \wedge x_{2}\right]=1$. In other words,

$$
x_{2}=u \gamma_{k_{1}}^{*} \wedge \ldots \wedge \gamma_{k_{a}}^{*},
$$

where $\left\{i_{1}, \ldots, i_{g-a}\right\} \cup\left\{k_{1}, \ldots, k_{a}\right\}=[g]$ and

$$
u=\sigma\left(i_{1}, \ldots, i_{g-a}, k_{1}, \ldots, k_{a}\right)
$$

for $\sigma$ described in (1.29). Similarly, let $z_{1} \in \bigwedge^{b} F, z_{2} \in \bigwedge^{r-b} F$, and $z_{3} \in \bigwedge^{f-r} F$ be basis elements with

$$
z=z_{1} \wedge z_{2} \quad \text { and } \quad y=z_{2} \wedge z_{3} .
$$

The right side of (2.36) is equal to

$$
\left[z_{1} \wedge z_{2} \wedge z_{3}\right] \theta^{(p)}\left(x \otimes z_{2}\right) .
$$

The left side of (2.36) is equal to

$$
\begin{gathered}
(-1)^{(a+b)(r-b)} \theta^{(p)}\left(x \otimes z_{2}\right)\left[x_{2} \wedge z_{1} \wedge x \wedge z_{2} \wedge z_{3}\right] \\
=(-1)^{(a+b)(r-b+g-a)} \theta^{(p)}\left(x \otimes z_{2}\right)\left[x \wedge x_{2} \wedge z_{1} \wedge z_{2} \wedge z_{3}\right] .
\end{gathered}
$$

However, $r-b+g-a$ is even and

$$
\left[x \wedge x_{2} \wedge z_{1} \wedge z_{2} \wedge z_{3}\right]=\left[x \wedge x_{2}\right]\left[z_{1} \wedge z_{2} \wedge z_{3}\right]
$$

by (2.25). We conclude that both sides of (2.36) are equal to (2.37) and the proof is complete.

If $F_{j}$ is the module in position $j$ of the complex $\mathbb{F}$, then $F_{j}^{*}$ is in position $-j$ in the complex $\mathbb{F}^{*}$. If $a$ is an integer, then the complex $\mathbb{F}[a]$ is the complex $\mathbb{F}$ shifted to the right by $a$. In other words, $\mathbb{F}[a]_{j}=F_{j+a}$.

Remark 2.38. We have not yet shown that each $\mathfrak{D}^{q}$ is a complex. However, as soon as this fact has been established in Corollary 3.18, then we may conclude, from Proposition 2.23, that there is an isomorphism of complexes:

$$
\mathfrak{D}^{q} \cong\left(\mathfrak{D}^{f-2-q}\right)^{*}[-f]
$$

Indeed, the isomorphism $\mathfrak{D}_{\text {left }}^{q} \rightarrow\left(\mathfrak{D}_{\text {right }}^{f-2-q}\right)^{*}[-f]$ can be concatenated with the dual of the isomorphism $\mathfrak{D}_{\text {left }}^{f-2-q} \rightarrow\left(\mathfrak{D}_{\text {right }}^{q}\right)^{*}[-f]$ because of $(2.32)$.

The final result in this section provides an alternate description for our modules $K_{i j}$. Unlike Definition 2.15 (a), the description (2.42) has the same form for all $i$. 
We also are able to relate the $K_{i j}$ to the Buchsbaum Eisenbud free $R$-modules $L_{p}^{q}$; and as a consequence, we can calculate the rank of each $K_{i j}$. We first recall the definition of the module $L_{p}^{q}$. Let $G$ be a free $R$-module of rank $g \geq 0$. Consider the Koszul algebra $\left(S \bullet G \otimes \wedge^{\bullet} G, \partial\right)$ associated to the identity map on $G$ as described in (1.8). For all integers $p$ and $q$, Buchsbaum and Eisenbud [13, pg. 260] defined $L_{p}^{q}(G)$ to be the kernel of

$$
\partial: S_{p} G \otimes \bigwedge^{q-1} G \rightarrow S_{p+1} G \otimes \bigwedge^{q-2} G
$$

and they proved [13, Proposition 2.5] that $L_{p}^{q}(G)$ is a free $R$-module. If $0 \leq p$ and $1 \leq q \leq g+1$, then

$$
\operatorname{rank} L_{p}^{q}(G)=\left(\begin{array}{c}
g+p-1 \\
q+p-1
\end{array}\right)\left(\begin{array}{c}
q+p-2 \\
q-1
\end{array}\right)
$$

Observe that $L_{p}^{q}(G)=0$ if $p<0$ or $q \leq 0$ or $g+2 \leq q$. Furthermore,

$$
L_{0}^{q}(G)=\left\{\begin{array}{ll}
R & \text { if } q=1 \\
0 & \text { otherwise }
\end{array} \quad \text { and } \quad L_{p}^{g+1}(G)=\left\{\begin{array}{ll}
R & \text { if } g=p=0 \\
0 & \text { otherwise }
\end{array} .\right.\right.
$$

Lemma 2.40. Let $F$ and $G$ be free $R$-modules of rank $f$ and $g$ respectively, and let $\pi: E=G \oplus F^{*} \rightarrow G$ be the projection map. If $\left(S_{\bullet} G \otimes \wedge^{\bullet} E, \partial\right)$ is the Koszul algebra associated to $\pi$, then for all integers $a$ and $b$, the kernel of

$$
\partial: S_{a} G \otimes \bigwedge^{b} E \rightarrow S_{a+1} G \otimes \bigwedge^{b-1} E
$$

is isomorphic to the free $R$-module

$$
\bigoplus_{v=0}^{f} L_{a}^{b-f+v+1}(G) \otimes \bigwedge^{v} F
$$

Proof. The indicated map decomposes into the direct sum

$$
\bigoplus_{v=0}^{f}\left(\partial: S_{a} G \otimes \bigwedge^{b-v} G \rightarrow S_{a+1} G \otimes \bigwedge^{b-v-1} G\right) \otimes \bigwedge^{v} F^{*}
$$

therefore, the kernel is

$$
\bigoplus_{v=0}^{f} L_{a}^{b-v+1}(G) \otimes \bigwedge^{v} F^{*}
$$

Replace $v$ by $f-v$ and use (1.6) to obtain the conclusion. 


\section{Proposition 2.41.}

(a) All of the modules $L_{i j}, K_{i j}$, and $Q_{i}$ are free $R$-modules.

(b) The module $K_{i j}$ is isomorphic to

$$
\bigoplus_{v=0}^{f} L_{i+1}^{g-j+v}(G) \otimes \bigwedge^{v} F
$$

Proof.

(a) It is clear that the $Q_{i}$ are free modules. The modules $K_{i j}$ are free because of (b). We know, from $(2.24)$, that $L_{i j} \cong\left(K_{i e-j}\right)^{*}$; and therefore, the $L_{i j}$ are also free.

(b) Let $\left(S_{\bullet} G \otimes \bigwedge^{\bullet} E, \partial\right)$ be the Koszul algebra associated to the projection map

$$
\pi: E=G \oplus F^{*} \rightarrow G
$$

For all integers $i$ and $j$, let

$$
Z_{i j}=\operatorname{Ker}\left(\partial: S_{i+1} G \otimes \bigwedge^{e-j-1} E \rightarrow S_{i+2} G \otimes \bigwedge^{e-j-2} E\right)
$$

Claim (b) follows from Lemma 2.40 as soon as we show that

$$
K_{i j} \cong Z_{i j}
$$

for all integers $i$ and $j$. It is well known that all of the complexes $\mathfrak{C}^{q}$ (see $(0.1)$ ), which are associated to the map $\pi$, are split exact. It follows that

$$
\begin{aligned}
& S_{i-1} G \otimes \bigwedge^{e-j+1} E \stackrel{\partial}{\rightarrow} S_{i} G \otimes \bigwedge^{e-j} E \rightarrow Z_{i j} \rightarrow 0, \quad \text { for } \quad i>0, \quad \text { and } \\
& D_{0} G^{*} \otimes \bigwedge^{e-j+g} E \stackrel{c}{\rightarrow} S_{0} G \otimes \bigwedge^{e-j} E \rightarrow Z_{0 j} \rightarrow 0
\end{aligned}
$$

are exact sequences, where $c$ is described in $(0.2)$.

We first prove (2.42) for $i=0$. The module $\bigwedge^{e-j+g} E$ is the direct sum

$$
\sum_{a+b=e-j+g} \bigwedge^{a} G \otimes \bigwedge^{b} F^{*}
$$

The map $c$ carries $\bigwedge^{g} G \otimes \bigwedge^{e-j} F^{*}$ isomorphically onto the summand $\bigwedge^{0} G \otimes$ $\bigwedge^{e-j} F^{*}$ of $\bigwedge^{e-j} E$. All of the other summands in (2.43) are in the kernel of $c$. Thus, $Z_{0 j}$ is the cokernel

$$
\bigwedge^{0} G \otimes \bigwedge^{e-j} F^{*} \hookrightarrow \bigwedge^{e-j} E \rightarrow Z_{0 j} \rightarrow 0
$$


The isomorphism $\beta: \bigwedge^{e-j} E \rightarrow \bigwedge^{j} E^{*}$ is defined in (1.6). Observe that $f-(e-j)=$ $j-g$; consequently, $\beta: \bigwedge^{e-j} F^{*} \rightarrow \bigwedge^{j-g} F$ is also an isomorphism. It is also clear that the diagram

$$
\begin{array}{cc}
\bigwedge^{e-j} F^{*} \stackrel{\text { incl }}{\longrightarrow} \bigwedge^{e-j} E \longrightarrow Z_{0 j} \longrightarrow 0 \\
\cong \downarrow \beta & \cong \downarrow_{\beta} \\
\bigwedge^{j-g} F \stackrel{\lambda}{\longrightarrow} \bigwedge^{j} E^{*} \longrightarrow K_{0 j} \longrightarrow 0
\end{array}
$$

commutes up to sign; and therefore, $Z_{0 j} \cong K_{0 j}$.

For $i>0$, we prove (2.42) by showing that

$$
\begin{aligned}
S_{i-1} G & \otimes \bigwedge^{j-1} E^{*} \stackrel{\eta}{\longrightarrow} S_{i} G \otimes \bigwedge^{j} E^{*} \longrightarrow K_{i j} \longrightarrow 0 \\
& \cong \downarrow^{1 \otimes \beta} \quad \cong 1 \otimes \beta \\
S_{i-1} G & \otimes \bigwedge^{e-j+1} E \stackrel{\partial}{\longrightarrow} S_{i} G \otimes \bigwedge^{e-j} E \longrightarrow Z_{i j} \longrightarrow 0
\end{aligned}
$$

commutes up to sign. We think of $\eta$ in terms of a basis $\gamma_{1}, \ldots, \gamma_{g}$ for $G$ as is described in (2.19). For each integer $a$, with $1 \leq a \leq g$, we decompose $E$ into $R \gamma_{a} \oplus E_{a}$; in other words, let

$$
\begin{aligned}
& E_{a}=R \gamma_{1} \oplus \ldots \oplus \widehat{R \gamma_{a}} \oplus \ldots \oplus R \gamma_{g} \oplus F^{*}, \quad \text { and } \\
& E_{a}^{*}=R \gamma_{1}^{*} \oplus \ldots \oplus \widehat{R \gamma_{a}^{*}} \oplus \ldots \oplus R \gamma_{g}^{*} \oplus F .
\end{aligned}
$$

If $r$ is an integer, let $p_{a}: \bigwedge^{r} E \rightarrow \bigwedge^{r-1} E_{a}$ be the composition

$$
\bigwedge^{r} E=\left(R \gamma_{a} \otimes \bigwedge^{r-1} E_{a}\right) \oplus \bigwedge^{r} E_{a} \stackrel{\text { proj }}{\longrightarrow} R \gamma_{a} \otimes \bigwedge^{r-1} E_{a} \cong \bigwedge^{r-1} E_{a}
$$

where the last map takes $\gamma_{a} \otimes x$ to $x$. Now we consider (2.44). Take $x \in S_{i-1} G$ and $y \in \bigwedge^{j-1} E^{*}$. It is clear that

$$
\begin{aligned}
& (1 \otimes \beta) \eta(x \otimes y)=\sum_{a=1}^{g} \gamma_{a} x \otimes \beta\left(\gamma_{a}^{*} \wedge y\right), \quad \text { and } \\
& \partial(1 \otimes \beta)(x \otimes y)=\sum_{a=1}^{g} \gamma_{a} x \otimes p_{a} \beta(y) .
\end{aligned}
$$

It suffices to show that

$$
p_{a} \beta(y)=(-1)^{e-j} \beta\left(\gamma_{a}^{*} \wedge y\right)
$$

for all integers $a$ and $j$ with $1 \leq a \leq g$. If $y \in R \gamma_{a}^{*} \otimes \bigwedge^{j-2} E^{*}$, then it is clear that both sides of (2.45) are zero. Assume that $y \in \bigwedge^{j-1} E_{a}^{*}$. Then

$$
\beta(y)=\gamma_{a} \wedge y^{\prime} \quad \text { for some } \quad y^{\prime} \in \bigwedge^{e-j} E_{a}
$$


(In particular, $p_{a} \beta(y)=y^{\prime}$.) Let $\langle\langle\rangle\rangle:, \bigwedge^{r} E^{*} \otimes \bigwedge^{r} E \rightarrow R$ be the perfect pairing of (1.2). We compare the terms in (2.45) by comparing

$$
\left\langle\left\langle-, y^{\prime}\right\rangle\right\rangle \text { and }\left\langle\left\langle-, \beta\left(\gamma_{a}^{*} \wedge y\right)\right\rangle\right\rangle: \bigwedge^{e-j} E^{*} \rightarrow R
$$

Both of these maps send $R \gamma_{a}^{*} \otimes \bigwedge^{e-j-1} E^{*}$ to zero. Let $z \in \bigwedge^{e-j} E_{a}^{*}$. Use the definition of $\langle\langle\rangle$,$\rangle together with (2.46)$ to see that

$$
\left\langle\left\langle z, p_{a} \beta(y)\right\rangle\right\rangle=\left\langle\left\langle z, y^{\prime}\right\rangle\right\rangle=\left\langle\left\langle\gamma_{a}^{*} \wedge z, \gamma_{a} \wedge y^{\prime}\right\rangle\right\rangle=\left\langle\left\langle\gamma_{a}^{*} \wedge z, \beta(y)\right\rangle\right\rangle .
$$

On the other hand, the definition of $\beta$ in (1.7) shows that

$\left\langle\left\langle\gamma_{a}^{*} \wedge z, \beta(y)\right\rangle\right\rangle=\left[\left(\gamma_{a}^{*} \wedge z\right) \wedge y\right]=(-1)^{e-j}\left[z \wedge\left(\gamma_{a}^{*} \wedge y\right)\right]=(-1)^{e-j}\left\langle\left\langle z, \beta\left(\gamma_{a}^{*} \wedge y\right)\right\rangle\right\rangle$

hence, $(2.45)$ is established and the proof is complete. 


\section{Section 3. Each $\mathfrak{D}^{q}$ IS A COMPlex.}

Given the data of (2.3) we have defined a family $\left\{\mathfrak{D}^{q}\right\}$ where each $\mathfrak{D}^{q}$ is the sequence of modules and maps of (2.5). We will have shown that each $\mathfrak{D}^{q}$ is a complex as soon as we show that (2.11) is a complex, and this is accomplished by proving the following pfaffian identity. Let $X$ be any $g \times g$ alternating matrix. If $n_{1}, \ldots, n_{m}$ is a list of integers (between 1 and $g$ ), then let

$X_{n_{1} \ldots n_{m}}$ represent the pfaffian of the principal submatrix of $X$ consisting of rows and columns $n_{1}, \ldots, n_{m}$ (in that order).

(The appropriate convention when $m=0$ is to set $X_{\text {empty list }}=1$.) In Proposition 3.4 we prove that if $A, B, C$, and $D$ are index sets of size $a, b, c$, and $d$, respectively, with $a+b+d$ even and $a+b \leq d-2$, then

$$
\sum_{t} \sum_{\substack{E \cup F=D \\|E|=t}}(-1)^{(a+c+t) / 2} \sigma(E F) X_{A C E} X_{B C F}=0 .
$$

The first sum is taken over all integers $t$ such that $a+c+t$ is even. (See (1.30) and (1.29) for the meaning of the second sum and the meaning of $\sigma(E F)$.) We make sure that the notation is clear by recording two trivial applications of (3.2). If $a=b=c=0$ and $D=\{1,2\}$, then (3.2) becomes the identity:

$$
-X_{12} \cdot 1+1 \cdot X_{12}=0 .
$$

If $A=\{1\}, b=c=0$, and $D=\{2,3,4\}$, then (3.2) becomes the (more interesting) identity:

$$
-X_{12} X_{34}+X_{13} X_{24}-X_{14} X_{23}+X_{1234} \cdot 1=0 .
$$

We prove and apply (3.2) in a coordinate free manner.

Definition 3.3. Let $G$ be a free module of rank $g$ over the commutative noetherian ring $R$; let $\xi: G^{*} \rightarrow G$ be an alternating map, and let $a, b, c$, and $d$ be integers with $a+b+d$ even. For every choice of $u$ in $\bigwedge^{c} G^{*}$, we define the map $\zeta_{u}: \bigwedge^{a} G^{*} \otimes$ $\bigwedge^{b} G^{*} \otimes \bigwedge^{d} G^{*} \rightarrow R$ to be the composition:

$$
\bigwedge^{a} G^{*} \otimes \bigwedge^{b} G^{*} \otimes \bigwedge^{d} G^{*} \stackrel{1 \otimes 1 \otimes \Delta}{\longrightarrow} \sum_{t} \bigwedge^{a} G^{*} \otimes \bigwedge^{b} G^{*} \otimes \bigwedge^{t} G^{*} \otimes \bigwedge^{d-t} G^{*} \stackrel{\sum f_{t}}{\longrightarrow} R
$$

where $t$ varies over all integers between 0 and $d$ with $a+t+c$ even, and

$$
f_{t}: \bigwedge^{a} G^{*} \otimes \bigwedge^{b} G^{*} \otimes \bigwedge^{t} G^{*} \otimes \bigwedge^{d-t} G^{*} \rightarrow R
$$

is defined by

$$
f_{t}(x \otimes y \otimes z \otimes w)=(-1)^{p} \xi^{(p)}(x \otimes u \otimes z) \xi^{(q)}(y \otimes u \otimes w)
$$

for $p=(a+c+t) / 2$ and $q=(b+c+d-t) / 2$. (The $p^{t h}$ divided power, $\xi^{(p)}$, of $\xi$ is defined above Observation 1.19.) 
Proposition 3.4. In the notation of Definition 3.3, if $a+b \leq d-2$, then the map $\zeta_{u}$ is identically zero for all choices of $u$.

Proof. We first reduce to the case where $R$ is a field, and a basis for $G$ has been chosen so that the matrix of $\xi$ is

$$
X=\left[\begin{array}{rrrrr}
0 & 1 & \cdots & 1 & 1 \\
-1 & 0 & \cdots & 1 & 1 \\
\vdots & \vdots & & \vdots & \vdots \\
-1 & -1 & \cdots & 0 & 1 \\
-1 & -1 & \cdots & -1 & 0
\end{array}\right]
$$

(The above matrix has 0 along the main diagonal, 1 in every entry above the diagonal, and -1 in every entry below the diagonal.) Indeed, every ring is a $\mathbb{Z}$ algebra, so we may first assume that $R$ is the polynomial ring $\mathbb{Z}[X]$ where the generic alternating matrix $X$ represents $\xi$. Furthermore, a map $\zeta_{u}: G \rightarrow R$ is identically zero if and only if $\zeta_{u} \otimes Q$ is identically zero where $Q$ is the quotient field of $R$. Finally, the map $\xi \otimes Q$ has rank $g$ if $g$ is even; or rank $g-1$, if $g$ is odd; consequently, there is a basis for $G$ so that the matrix of $\xi \otimes Q$ is given in (3.5). The first reduction is complete.

The pfaffian of every even-sized matrix which has the form of (3.5) is +1 . (This fact may be established by induction using the Laplace expansion in (1.18).) We have already chosen a basis $\gamma_{1}, \ldots, \gamma_{g}$ for $G$ and the corresponding dual basis $\gamma_{1}^{*}, \ldots, \gamma_{g}^{*}$ for $G^{*}$. If $A=\left(n_{1}, \ldots, n_{a}\right)$ is an $a$-tuple of integers, then, as in (1.31), we let

$$
\gamma_{A}^{*}=\gamma_{n_{1}}^{*} \wedge \gamma_{n_{2}}^{*} \wedge \ldots \wedge \gamma_{n_{a}}^{*} .
$$

If $a=2 p$, then it is clear that

$$
\xi^{(p)}\left(\gamma_{A}^{*}\right)=X_{A}=\sigma(A) .
$$

(The notation $X_{A}$ is explained in (3.1) and the meaning of $\sigma$ is given in (1.29).) We next show that it suffices to prove:

If $A, B, C$, and $D$ are disjoint index sets of size $a, b, c$, and $d$, respectively, with $a+b+d$ even and $a+b \leq d-2$, then $\zeta_{u}\left(\gamma_{A}^{*} \otimes \gamma_{B}^{*} \otimes \gamma_{D}^{*}\right)=0$ for $u=\gamma_{C}^{*}$.

If the word "disjoint" is removed from (3.8), then it is obvious that (3.8) is equivalent to the result we want to prove. In this part of the argument, we show that if $A, B, C$, and $D$ are arbitrary index sets (with $a+b+d$ even and $a+b \leq d-2$ ), then $\zeta_{u}\left(\gamma_{A}^{*} \otimes \gamma_{B}^{*} \otimes \gamma_{D}^{*}\right)$ is equal to something which we show to be zero when we establish (3.8). Indeed, if $C$ has an index in common with $A, B$, or $D$, then $\zeta_{u}\left(\gamma_{A}^{*} \otimes \gamma_{B}^{*} \otimes \gamma_{D}^{*}\right)$ is obviously zero. If $A$ and $B$ have an index $i$ in common, then we remove it from $A$ and $B$ and place it in $C$. Observe that

$$
\zeta_{u}\left(\gamma_{A}^{*} \otimes \gamma_{B}^{*} \otimes \gamma_{D}^{*}\right)= \pm \zeta_{u^{\prime}}\left(\gamma_{A^{\prime}}^{*} \otimes \gamma_{B^{\prime}}^{*} \otimes \gamma_{D^{\prime}}^{*}\right)
$$


where $u^{\prime}=\gamma_{C^{\prime}}^{*}, A^{\prime}=A \backslash\{i\}, B^{\prime}=B \backslash\{i\}, C^{\prime}=C \cup\{i\}$, and $D^{\prime}=D$. It is important to notice that $(a-1)+(b-1) \leq d-2$. If $A$ and $D$ have an index $i$ in common, then we remove $i$ from $A$ and $D$ and place it in $C$. Observe that (3.9) continues to hold (for $A^{\prime}, B^{\prime}, C^{\prime}$, and $D^{\prime}$ appropriately defined) and that

$$
(a-1)+b \leq(d-1)-2 .
$$

We have successfully reduced the problem to proving (3.8). Let $A, B, C$, and $D$ be as described in (3.8). Observe, by way of (3.7), that

$$
\zeta_{u}\left(\gamma_{A}^{*} \otimes \gamma_{B}^{*} \otimes \gamma_{D}^{*}\right)=\sum_{\substack{t \\ a+c+t \text { even }}} \sum_{\substack{E \cup F=D \\|E|=t}}(-1)^{(a+c+t) / 2} \sigma(E F) \sigma(A C E) \sigma(B C F)
$$

We use two formulas about arrangements of index sets. Suppose that $S, T$ and $U$ are index sets. A straightforward induction on the size of $S$ shows that

$$
\sigma(S T U)=\sigma(S T) \sigma(S U) \sigma(T U)
$$

Furthermore, if $V$ is an index set and $T \cup U$ is a partition of $V$ into two disjoint subsets, then one can rearrange $S T U$ into ascending order by first arranging $T U$ to be $V$ and then arranging $S V$. It follows that

$$
\sigma(S T U)=\sigma(S V) \sigma(T U)
$$

We apply (3.10) and (3.11) in order to see that $\zeta_{u}\left(\gamma_{A}^{*} \otimes \gamma_{B}^{*} \otimes \gamma_{D}^{*}\right)$ is equal to

$$
\sigma(A C) \sigma(B C) \sigma(C D)\left[\sum_{\substack{t \\ a+c+t \text { even }}} \sum_{\substack{E \cup F=D \\|E|=t}}(-1)^{(a+c+t) / 2} \sigma(E F) \sigma(A E) \sigma(B F)\right]
$$

The sum inside the bracket is zero by the following combinatorial lemma.

Lemma 3.12. Let $A, B$, and $D$ be disjoint index sets of size $a, b$, and $d$, respectively, and let $c$ be a fixed integer. Define

$$
\Psi(A, B, D)=\sum_{\substack{t \\ a+c+t \text { even }}} \sum_{\substack{E \cup F=D \\|E|=t}}(-1)^{(a+c+t) / 2} \sigma(E F) \sigma(A E) \sigma(B F) .
$$

If $a+b \leq d-2$, then $\Psi(A, B, D)=0$.

Proof. Let $D$ be $n_{1}<n_{2}<\ldots<n_{d}$ and define

$$
\Omega(A, B, D)=\operatorname{card}\left\{i \mid \exists x \in A \cup B \quad \text { with } \quad n_{i}<x<n_{i+1}\right\} .
$$

It is obvious that $\Omega(A, B, D) \leq a+b$. We will prove that

$$
\Omega(A, B, D) \leq d-2 \Rightarrow \Psi(A, B, D)=0
$$


by induction on $d$. We first suppose that $d=2$. If $a+c$ is even, then $\Psi(A, B, D)$ is equal to

$$
(-1)^{(a+c) / 2}[\sigma(D) \sigma(A) \sigma(B D)-\sigma(D) \sigma(A D) \sigma(B)]
$$

and this is zero because $\sigma(B D)=1=\sigma(A D)$ since $\Omega(A, B, D)=0$ and $d$ is even. If $a+c$ is odd, then $\Psi(A, B, D)$ is equal to

$$
(-1)^{(1+a+c) / 2}\left[\sigma\left(n_{1} n_{2}\right) \sigma\left(A n_{1}\right) \sigma\left(B n_{2}\right)+\sigma\left(n_{2} n_{1}\right) \sigma\left(A n_{2}\right) \sigma\left(B n_{1}\right)\right]
$$

This is also zero since $\sigma\left(A n_{1}\right)=\sigma\left(A n_{2}\right)$ and $\sigma\left(B n_{1}\right)=\sigma\left(B n_{2}\right)$ because $\Omega(A, B, D)=$ 0 .

Now we suppose that $d \geq 3$. Observe that $\Psi(A, B, D)$ is equal to

(3.14) $\sigma\left(A n_{1}\right) \Psi\left(A \cup\left\{n_{1}\right\}, B, D \backslash\left\{n_{1}\right\}\right)+(-1)^{a+c} \sigma\left(B n_{1}\right) \Psi\left(A, B \cup\left\{n_{1}\right\}, D \backslash\left\{n_{1}\right\}\right)=$

$(-1)^{d-1} \sigma\left(A n_{d}\right) \Psi\left(A \cup\left\{n_{d}\right\}, B, D \backslash\left\{n_{d}\right\}\right)+(-1)^{d+a+c-1} \sigma\left(B n_{d}\right) \Psi\left(A, B \cup\left\{n_{d}\right\}, D \backslash\left\{n_{d}\right\}\right)$.

If there is an $x$ in $A \cup B$ with $n_{1}<x<n_{2}$, then let

$$
\left(A^{\prime}, B^{\prime}, D^{\prime}\right) \quad \text { be either } \quad\left(A \cup\left\{n_{1}\right\}, B, D \backslash\left\{n_{1}\right\}\right) \quad \text { or } \quad\left(A, B \cup\left\{n_{1}\right\}, D \backslash\left\{n_{1}\right\}\right) \text {. }
$$

If there is an $x$ in $A \cup B$ with $n_{d-1}<x<n_{d}$, then let

$$
\left(A^{\prime}, B^{\prime}, D^{\prime}\right) \quad \text { be either } \quad\left(A \cup\left\{n_{d}\right\}, B, D \backslash\left\{n_{d}\right\}\right) \quad \text { or } \quad\left(A, B \cup\left\{n_{d}\right\}, D \backslash\left\{n_{d}\right\}\right) \text {. }
$$

In either case we see that $\Omega\left(A^{\prime}, B^{\prime}, D^{\prime}\right)=\Omega(A, B, D)-1$; consequently

$$
2 \leq|D|-\Omega(A, B, D)=\left|D^{\prime}\right|-\Omega\left(A^{\prime}, B^{\prime}, D^{\prime}\right)
$$

We know that $\Psi\left(A^{\prime}, B^{\prime}, D^{\prime}\right)=0$ by induction on $d$. It follows from (3.14) that $\Psi(A, B, D)$ is also zero. If there does not exist an $x$ in $A \cup B$ with $n_{1}<x<n_{2}$ or $n_{d-1}<x<n_{d}$, then it is clear that $\Omega(A, B, D) \leq d-3$. Let $\left(A^{\prime}, B^{\prime}, D^{\prime}\right)$ be as in (3.15). Observe that

$$
2=3-1 \leq(d-1)-\Omega(A, B, D)=\left|D^{\prime}\right|-\Omega\left(A^{\prime}, B^{\prime}, D^{\prime}\right) .
$$

Once again $\Psi\left(A^{\prime}, B^{\prime}, D^{\prime}\right)=0$ by induction on $d$; hence $\Psi(A, B, D)$ is also zero, and the proof is complete.

Corollary 3.16. Adopt the data of (2.3). Let $a, b$, and $d$ be integers with $a+b+d$ even and $2 g+2 \leq a+b+d$, and let $\zeta: \bigwedge^{a} G^{*} \otimes \bigwedge^{b} G^{*} \otimes \bigwedge^{d} F \rightarrow R$ be the map

$$
\bigwedge^{a} G^{*} \otimes \bigwedge^{b} G^{*} \otimes \bigwedge^{d} F \stackrel{1 \otimes 1 \otimes \Delta}{\longrightarrow} \sum_{t} \bigwedge^{a} G^{*} \otimes \bigwedge^{b} G^{*} \otimes \bigwedge^{t} F \otimes \bigwedge^{d-t} F \stackrel{\sum f_{t}}{\longrightarrow} R
$$

where $t$ varies over all integers with $a+t$ even and

$$
f_{t}: \bigwedge^{a} G^{*} \otimes \bigwedge^{b} G^{*} \otimes \bigwedge^{t} F \otimes \bigwedge^{d-t} F \longrightarrow R
$$


is defined $f_{t}(x \otimes y \otimes z \otimes w)=(-1)^{p} \theta^{(p)}(x \otimes z) \theta^{(q)}(y \otimes w)$ for $p=(a+t) / 2$ and $q=(b+d-t) / 2$. Then the map $\zeta$ is identically zero.

Proof. Let $A$ and $B$ be index sets of size $a$ and $b$ respectively. It suffices to show that $\zeta\left(\gamma_{A}^{*} \otimes \gamma_{B}^{*} \otimes-\right)$ is identically zero. (See (3.6).) Let $C=A \cap B, A^{\prime}=A \backslash C, B^{\prime}=$ $B \backslash C$, and $c=|C|$. Observe that $\zeta\left(\gamma_{A}^{*} \otimes \gamma_{B}^{*} \otimes-\right)= \pm \zeta_{u}\left(\gamma_{A^{\prime}}^{*} \otimes \gamma_{B^{\prime}}^{*} \otimes-\right)$ for $u=\gamma_{C}^{*}$ where $\zeta_{u}$ is defined in (3.3) for the alternating map $\theta: E^{*} \rightarrow E$. Since $A$ and $B$ are both subsets of $[g]$ (as defined in (1.28)), it is clear that

$$
a+b=|A \cup B|+|A \cap B| \leq g+c ;
$$

consequently,

$$
\begin{gathered}
2 a+2 b \leq 2 g+2 c, \quad \text { and } \\
\left|A^{\prime}\right|+\left|B^{\prime}\right|=a+b-2 c \leq 2 g-a-b .
\end{gathered}
$$

On the other hand, $2 g-a-b \leq d-2$ by hypothesis. We conclude, from Proposition 3.4 , that $\zeta$ is identically zero.

Observation 3.17. If $\theta: E^{*} \rightarrow E$ is the map of (2.3), then the rank of $\theta$ is at most $2 g$.

Proof. It is easy to see that the image of the restriction of $\bigwedge^{a+b} \theta$ to $\bigwedge^{a} G^{*} \otimes \bigwedge^{b} F$ is contained in $\sum_{i=0}^{a} \bigwedge^{b+i} G \otimes \bigwedge^{a-i} F^{*}$. The conclusion follows because the rank of $G$ is $g$.

Corollary 3.18. Each $\mathfrak{D}^{q}$ of (2.5) is a complex.

Proof. It suffices to show that (2.11) is a complex. Fix integers $a, b, c$, and $d$ with $a$ not equal to $0, c \neq g$,

$$
a+b=g+q+2, \quad \text { and } \quad c+d=q .
$$

We show that the component

$$
\bigwedge^{a} G^{*} \otimes \bigwedge^{b} F \stackrel{d}{\rightarrow} Q_{q} \stackrel{d}{\rightarrow} \bigwedge^{c} G^{*} \otimes \bigwedge^{d} F
$$

of (2.11) is a complex. Fix index sets $A, B, C$, and $D$ of size $a, b, c$, and $d$, respectively. Expand $d d\left(\gamma_{A}^{*} \otimes \phi_{B}\right)$ in terms of the standard basis of $\bigwedge^{c} G^{*} \otimes \bigwedge^{d} F$. It is easy to see that the coefficient of $\gamma_{C}^{*} \otimes \phi_{D}$, in this expansion, is zero unless $D \subseteq B$. Let $I$ be an arbitrary index set of size $b-d$, and let $\alpha$ be the coefficient of $\gamma_{C}^{*} \otimes \phi_{D}$ in the expansion of $d d\left(\gamma_{A}^{*} \otimes \phi_{I} \wedge \phi_{D}\right)$. The proof is complete once we show that $\alpha$ equals zero. Let $H$ be the complement of $C$ in $[g]$. It is not difficult to see that

$$
\alpha= \pm \sum_{r}(-1)^{(g+r-q) / 2} \sum_{\substack{J \cup K=I \\|J|=b-r}} \sigma(J K) \theta^{(\cdot)}\left(\gamma_{A}^{*} \otimes \phi_{J}\right) \theta^{(\cdot)}\left(\gamma_{H}^{*} \otimes \phi_{K}\right)
$$

where $r$ varies over all integers which satisfy $(2.16)$. (The first $(\cdot)$ means $(a+b-r) / 2$ and the second $(\cdot)$ means $(g-c+r-d) / 2$.) Let $t=b-r$, and use (3.19) to see that

$$
\left.\alpha= \pm \sum_{t}(-1)^{(t+a) / 2} \sum_{\substack{J \cup K=I \\|J|=t}} \sigma(J K) \theta^{(\cdot)}\left(\gamma_{A}^{*} \otimes \phi_{J}\right) \theta^{(\cdot)}\left(\gamma_{H}^{*} \otimes \phi_{K}\right)\right)
$$


where $t$ varies over all integers with $t+a$ even and

$$
2-a \leq t \leq 2 g-a
$$

Observe that (3.20) actually does not put any restriction on $t$. Indeed, $2-a \leq t$ follows automatically from the conditions $1 \leq a$ and $t+a$ even. Furthermore, the rank of $\theta$ is at most $2 g$ by Observation 3.17; consequently, $\theta^{((t+a) / 2)}$ is identically zero for $t>2 g-a$. Use (3.19) in order to see that

$$
a+|H|+|I|=a+(g-c)+(b-d)=2 g+2 .
$$

The proof is completed by appealing to Corollary 3.16 . 


\section{Section 4. Elementary facts about the Complexes $\left\{\mathfrak{D}^{q}\right\}$.}

The notation of (2.3) is in effect throughout this section. We collect some information about the complexes $\mathfrak{D}^{q}$ which follows quickly from their definition. Recall, from Remark 2.38, that

$$
\mathfrak{D}^{q} \cong\left(\mathfrak{D}^{f-2-q}\right)^{*}[-f] .
$$

Observation 4.2. Adopt the data of (2.3).

(a) If $i<0$, or $j<0$, or $e \leq j$, then $K_{i j}=0$.

(b) If $0 \leq i$ and $0 \leq j \leq e-1$, then $K_{i j} \neq 0$.

(c) If $i<0$, or $j \leq 0$, or $e<j$, then $L_{i j}=0$.

(d) If $0 \leq i$ and $1 \leq j \leq e$, then $L_{i j} \neq 0$.

(e) If $q \leq-1-g$, or $e-1 \leq q$, then $Q_{q}=0$.

(f) If $1 \leq f$ and $-g \leq q \leq e-2$, then $Q_{q} \neq 0$.

(g) If $f=0$ and $-g \leq q \leq e-2$, then

$$
\left\{\begin{array}{l}
Q_{q}=0 \quad \text { if } q+g \text { is odd, and } \\
Q_{q} \neq 0 \quad \text { if } q+g \text { is even. }
\end{array}\right.
$$

Proof. All of the assertions follow quickly from Definition 2.15. We give the proof of (f) as an example. If $g-2 \leq q \leq e-2$, then $\bigwedge^{r} F$ is a non-zero summand of $Q_{q}$ for $r=q-g+2$. If $1-g \leq q \leq g-2$, then $\bigwedge^{r} F$ is a non-zero summand of $Q_{q}$ for $r$ equal to 0 or 1 . If $q=-g$, then $Q_{q} \cong Q_{e-2}^{*}$ by (2.32) and therefore, $Q_{q}$ also is non-zero.

A portrait of the family $\left\{\mathfrak{D}^{q}\right\}$ may be found in Figure 4.4. If $\mathbb{F}$ is a complex for which there are integers $a$ and $b$ with $0 \leq b$ such that $F_{j}=0$ if $j<a, F_{j}=0$ if $a+b<j$, and $F_{j} \neq 0$ if $a \leq j \leq a+b$, then we say that the length, $\ell(\mathbb{F})$, of $\mathbb{F}$ is $b$. (If every module in a complex is zero, then we don't assign that complex a length.) The length of the complex $\mathfrak{D}^{q}$ may be read from Observation 4.2 together with Figure 4.4. This information is summarized in Observation 4.3. (It is useful to remember that $\mathfrak{D}^{q}$ and $\mathfrak{D}^{f-2-q}$ have the same length because of (4.1).)

\section{Observation 4.3.}

(a) If $-1 \leq q$, then $\mathfrak{D}_{j}^{q}=0$ for all $j<0$.

(b) If $q \leq f-1$, then $\mathfrak{D}_{j}^{q}=0$ for all $j>f$.

(c) If $j \leq-g$ or $e \leq j$, then $\mathfrak{D}_{j}^{q}=0$ for all $q$.

(d) The length of $\mathfrak{D}^{q}$ is at most $e-1$ for all $q$. Furthermore, if $q \leq-g$ or $e-2 \leq q$, then $\mathfrak{D}^{q}$ has length $e-1$.

(e) If $f \geq 1$, then

$$
\ell\left(\mathfrak{D}^{q}\right)= \begin{cases}f & \text { if }-1 \leq q \leq f-1 \\ q+1 & \text { if } f-1 \leq q \leq e-2 \\ e-1 & \text { if } e-2 \leq q .\end{cases}
$$

(f) Assume that $f=0$ and $g$ is even. The module $\mathfrak{D}_{j}^{-1}$ is equal to zero for all integers $j$. If $q$ is odd and $1 \leq q \leq g-1$, then $\mathfrak{D}^{q}$ and $\mathfrak{D}^{q-1}$ both have length $q$. 
The FAMILY $\left\{\mathfrak{D}^{q}\right\}$ :

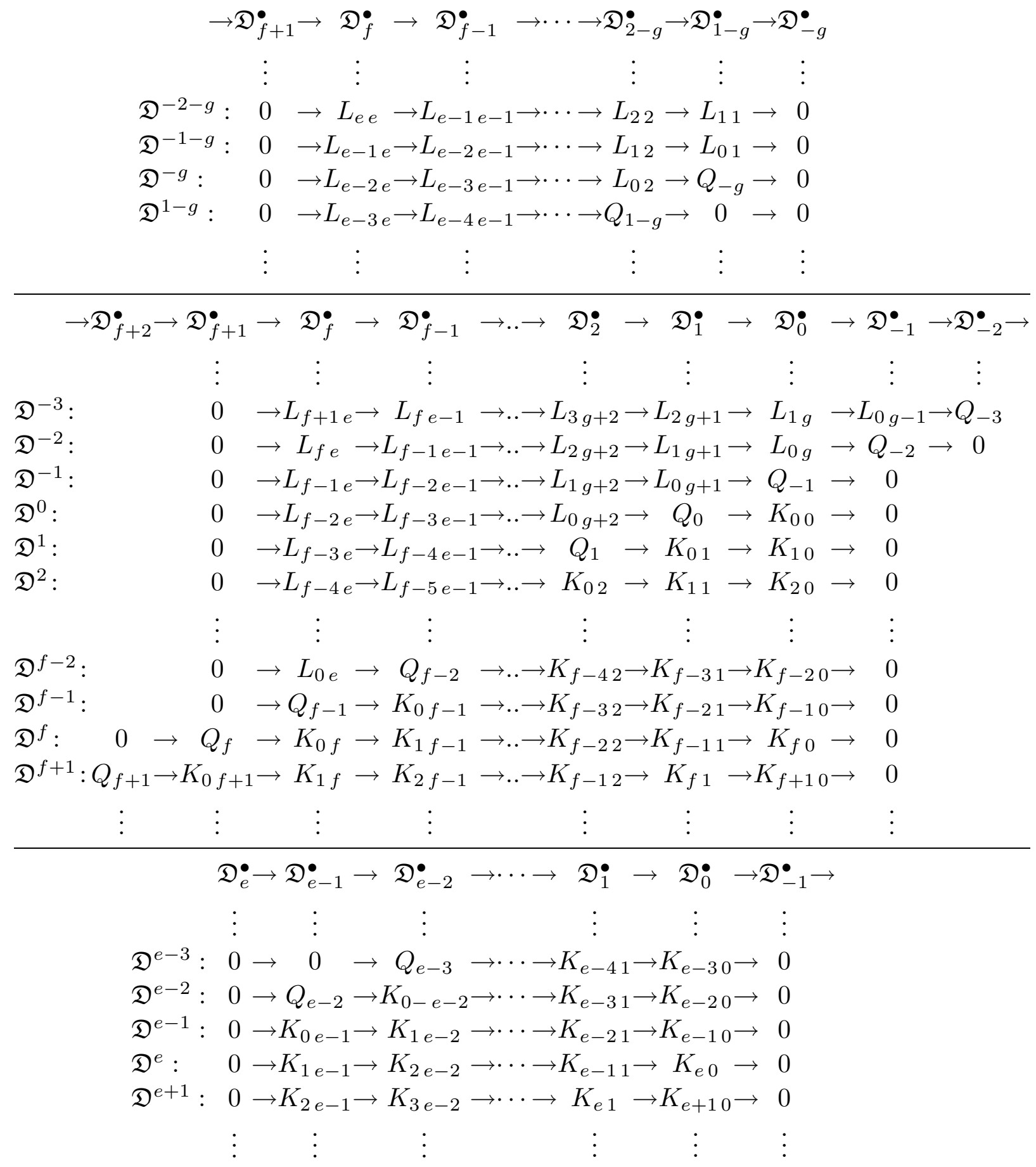

FiguRE 4.4

(g) Assume that $f=0$ and $g$ is odd. If $q$ is even and $0 \leq q \leq g-1$, then $\mathfrak{D}^{q}$ and $\mathfrak{D}^{q-1}$ both have length $q$.

Remark 4.5. If $f-1 \leq q \leq e-2$, then the length of $\mathfrak{D}^{q}$ grows with $q$. This growth is linear if $f$ is positive. However, this growth is a step function if $f=0$; see Figures 4.6 and 4.7 . 
THE COMPLEXES $\mathfrak{D}^{q}$ WHEN $f=0$ AND $g \geq 4$ IS EVEN:

$$
\begin{aligned}
& \rightarrow \mathfrak{D}_{4}^{\bullet} \rightarrow \mathfrak{D}_{3}^{\bullet} \rightarrow \mathfrak{D}_{2}^{\bullet} \rightarrow \mathfrak{D}_{1}^{\bullet} \rightarrow \mathfrak{D}_{0}^{\bullet} \rightarrow \mathfrak{D}_{-1}^{\bullet} \rightarrow \\
& \mathfrak{D}^{-2}: \quad 0 \rightarrow 0 \rightarrow 0 \rightarrow 0 \rightarrow L_{0 g} \rightarrow Q_{-2} \\
& \mathfrak{D}^{-1}: \quad 0 \rightarrow 0 \rightarrow 0 \rightarrow 0 \rightarrow 0 \rightarrow 0 \\
& \mathfrak{D}^{0}: \quad 0 \rightarrow 0 \rightarrow 0 \rightarrow Q_{0} \rightarrow K_{00} \rightarrow 0 \\
& \mathfrak{D}^{1}: \quad 0 \rightarrow 0 \rightarrow 0 \rightarrow K_{01} \rightarrow K_{10} \rightarrow 0 \\
& \mathfrak{D}^{2}: \quad 0 \rightarrow Q_{2} \rightarrow K_{02} \rightarrow K_{11} \rightarrow K_{20} \rightarrow 0 \\
& \mathfrak{D}^{3}: \quad 0 \rightarrow K_{03} \rightarrow K_{12} \rightarrow K_{21} \rightarrow K_{30} \rightarrow 0
\end{aligned}
$$

FiguRE 4.6

The COMPLEXes $\mathfrak{D}^{q}$ When $f=0$ AND $g \geq 3$ IS ODD:

$$
\begin{aligned}
& \rightarrow \mathfrak{D}_{3}^{\bullet} \rightarrow \mathfrak{D}_{2}^{\bullet} \rightarrow \mathfrak{D}_{1}^{\bullet} \rightarrow \mathfrak{D}_{0}^{\bullet} \rightarrow \mathfrak{D}_{-1}^{\bullet} \rightarrow \\
& \mathfrak{D}^{-2}: \quad 0 \rightarrow 0 \rightarrow 0 \rightarrow L_{0 g} \rightarrow 0 \\
& \mathfrak{D}^{-1}: \quad 0 \rightarrow 0 \rightarrow 0 \rightarrow Q_{-1} \rightarrow 0 \\
& \mathfrak{D}^{0}: \quad 0 \rightarrow 0 \rightarrow 0 \rightarrow K_{00} \rightarrow 0 \\
& \mathfrak{D}^{1}: \quad 0 \rightarrow Q_{1} \rightarrow K_{01} \rightarrow K_{10} \rightarrow 0 \\
& \mathfrak{D}^{2}: \quad 0 \rightarrow K_{02} \rightarrow K_{11} \rightarrow K_{20} \rightarrow 0
\end{aligned}
$$

FiguRE 4.7

If $(g, f)$ is the shape of an almost alternating map $\rho$, then $g \geq 1$ and $f \geq 0$. The complexes that occur at the boundary $f=0$ are recorded in Figures 4.6 and 4.7. These complexes are new and interesting. For example, if $\rho$ is sufficiently general and $g$ is odd, then these complexes resolve the powers of a grade three Gorenstein ideal; see Corollary 6.25. On the other hand, the complexes that occur at the boundary $g=1$ are very familiar. (We take advantage of this fact in some of our proofs by induction.)

Example 4.8. If $\rho=[0 \psi]$ is an almost alternating map of shape $(1, f)$, then for every integer $q, \mathfrak{D}^{q}$ is the ordinary Koszul complex associated to the map $\psi: F \rightarrow$ $G \cong R$.

Proof. Use Definition 2.15 to see that

$$
\begin{aligned}
K_{i j} & =S_{i} G \otimes \bigwedge^{j} F \cong \bigwedge^{j} F \\
Q_{j} & =\bigwedge^{j+1} F \\
L_{i j} & =D_{i} G^{*} \otimes \bigwedge^{1} G^{*} \otimes \bigwedge^{j-1} F \cong \bigwedge^{j-1} F
\end{aligned}
$$


for all integers $i$ and $j$. Furthermore, it is not difficult to see that the map $\mathfrak{D}_{j}^{q} \rightarrow$ $\mathfrak{D}_{j-1}^{q}$ is isomorphic to the composition:

$$
\bigwedge^{j} F \stackrel{\Delta}{\longrightarrow} F \otimes \bigwedge^{j-1} F \stackrel{\psi \otimes 1}{\longrightarrow} G \otimes \bigwedge^{j-1} F \stackrel{[] \otimes 1}{\longrightarrow} \bigwedge^{j-1} F
$$

We are next interested in studying the homology in position zero of the complexes $\left\{\mathfrak{D}^{q}\right\}$. We are immediately forced to consider various ideals associated to the data of (2.3). Pick bases for $F$ and $G$ as described in Remark 2.2 (c), and let

$$
T=\left[\begin{array}{cc}
X & Y \\
-Y^{\mathrm{t}} & 0
\end{array}\right]
$$

be the matrix of $\theta$ with respect to these bases. If $Y^{\prime}$ is a submatrix of $Y$ which consists of rows $1, \cdots, g$ and $r$ arbitrary columns then

$$
\left[\begin{array}{cc}
X & Y^{\prime} \\
-\left(Y^{\prime}\right)^{\mathrm{t}} & 0
\end{array}\right]
$$

is a principal submatrix of $T$ which contains $X$.

Definition 4.10. Let $\rho=\left[\begin{array}{ll}X & Y\end{array}\right]$ be an almost alternating matrix of shape $(g, f)$ over the commutative noetherian ring $R$, let $T$ be the matrix described in (4.9), and let $Y_{* j}$ represent the $j^{\text {th }}$ column of $Y$.

(a) Define $J=J(\rho)$ to be the $R$-ideal generated by the pfaffians of all principal submatrices of $T$ which contain $X$.

(b) If $g$ is odd, then define $I=I(\rho)$ to be the $R$-ideal $\operatorname{Pf}_{g-1}(X)$, and define $A=A(\rho)$ to be the $R$-ideal $\left(a_{1}, \cdots, a_{f}\right)$ where

$$
a_{j}=\operatorname{pf}\left[\begin{array}{c|c}
X & Y_{* j} \\
\hline-\left(Y_{* j}\right)^{\mathrm{t}} & 0
\end{array}\right] \text { for } 1 \leq j \leq f .
$$

(c) If $g$ is even and $f$ is positive, then define $I=I(\rho)$ to be the $R$-ideal

$$
\operatorname{Pf}_{g}\left[\begin{array}{c|c}
X & Y_{* 1} \\
\hline-\left(Y_{* 1}\right)^{\mathrm{t}} & 0
\end{array}\right]
$$

and define $A=A(\rho)$ to be the $R$-ideal $\left(\operatorname{pf}(X), a_{2}, \cdots, a_{f}\right)$ where

$$
a_{j}=p f\left[\begin{array}{c|cc}
X & Y_{* 1} & Y_{* j} \\
\hline-\left(Y_{* 1}\right)^{\mathrm{t}} & 0 & 0 \\
-\left(Y_{* j}\right)^{\mathrm{t}} & 0 & 0
\end{array}\right] \quad \text { for } \quad 2 \leq j \leq f .
$$

Remarks 4.11. Retain the notation of Definition 4.10.

(a) If $f=0$ and $g$ is even, then neither $I(\rho)$ nor $A(\rho)$ is defined. 
(b) The ideal $J$ is independent of the choice of bases. In fact, we know from Observation 1.19 that $J$ is the image of the map

$$
\sum_{r} \bigwedge^{g} G^{*} \otimes \bigwedge^{r} F \stackrel{\sum \theta^{((g+r) / 2)}}{\longrightarrow} R
$$

where $r$ varies over all the integers with $g+r$ even.

(c) If $g$ is odd, then $I(\rho)$ and $A(\rho)$ are also coordinate-free. If $g$ is even and $f$ is positive, then $I(\rho)$ and $A(\rho)$ depend on the choice of one basis element, $\phi_{1}$, from $F$; they do not depend on the rest of the basis for $F$ or the basis of $G$.

(d) The ideal $I$ has been defined to be the maximal order pfaffians of an odd-sized alternating matrix. It follows from (1.24) and Proposition 1.22 that if $I$ is a proper ideal, then grade $I \leq 3$. Furthermore, if grade $I=3$, then $I$ is perfect.

Examples 4.12. Let $\rho=\left[\begin{array}{ll}X & Y\end{array}\right]$ be an almost alternating matrix of shape $(g, f)$. If either of the parameters is small, then the ideal $J=J(\rho)$ is well understood.

(a) If $g=1$, then $Y$ is a $1 \times f$ matrix and $J$ is generated by the entries of $Y$. We saw in Example 4.8 that in this case every $\mathfrak{D}^{q}$ is simply the Koszul complex on the generators of $J$.

(b) If $g=2$, then $Y$ is a $2 \times f$ matrix,

$$
X=\left[\begin{array}{cc}
0 & x \\
-x & 0
\end{array}\right]
$$

and $J=\left(x, I_{2}(Y)\right)$. If the almost alternating matrix $\rho$ is generic, then $J$ is a hypersurface section of a determinantal ideal.

(c) Suppose $g=3$. If $X$ is the matrix

$$
\left[\begin{array}{ccc}
0 & x_{12} & x_{13} \\
-x_{12} & 0 & x_{23} \\
-x_{13} & -x_{23} & 0
\end{array}\right]
$$

then let $\mathbf{x}$ be the matrix $\left[\begin{array}{lll}x_{23} & -x_{13} & x_{12}\end{array}\right]$. The ideal $J$ is equal to $I_{1}(\mathbf{x} Y)+I_{3}(Y)$. Ideals of this form have been studied extensively in [7].

(d) If $f=0$ and $g$ is odd, then $J$ is the zero ideal.

(e) If, either $f=1$, or else, $f=0$ and $g$ is even, then $J$ is a principal ideal generated by the pfaffian of a single even-sized alternating matrix:

$$
J= \begin{cases}\operatorname{Pf}_{g}(X) & \text { if } g \text { is even } \\ \operatorname{Pf}_{g+1}(T) & \text { if } g \text { is odd }\end{cases}
$$

Proposition 4.13. Retain the notation of Definition 4.10, let $M=\operatorname{coker} \rho$, and let $\bar{R}$ be the ring $R / J$.

(a) The homology $H_{0}\left(\mathfrak{D}^{0}\right)$ is equal to $\bar{R}$.

(b) If $1 \leq q$, then $H_{0}\left(\mathfrak{D}^{q}\right) \cong S_{q}(M)$. 
(c) For each integer $q$ with $0 \leq q$, the homology $H_{0}\left(\mathfrak{D}^{q}\right)$ is an $\bar{R}$-module.

(d) If $g$ is odd or $f$ is positive, then the following statements hold.

(i) There is an $\bar{R}$-module surjection $M \rightarrow(I / A)$.

(ii) If grade $I \geq 3$, then $M \cong(I / A)$.

(iii) The ideals $I, J$, and $A$ satisfy $I J \subseteq A \subseteq(I \cap J)$.

(iv) For each integer $q \geq 0$ there is an $\bar{R}$ - module surjection $H_{0}\left(\mathfrak{D}^{q}\right) \rightarrow$ $I^{q} \bar{R}$.

(e) There is an ideal $\widetilde{J}$ of $R$ (defined in (4.19)) and an $R$-module surjection

$$
H_{0}\left(\mathfrak{D}^{-1}\right) \rightarrow \widetilde{J} / J .
$$

The objects of Proposition 4.13 For small $f$ or $g$ : The matrix $\widetilde{T}$ and the element $y_{1}$ are defined in (4.19).

\begin{tabular}{l|c|c|c|c|c|} 
& $J$ & $\bar{R}$ & $I$ & $\widetilde{J}$ & $H_{0}\left(\mathfrak{D}^{-1}\right)$ \\
\hline $\begin{array}{l}f=0 \\
g \text { odd }\end{array}$ & 0 & $R$ & $\operatorname{Pf}_{g-1}(X)$ & $(\operatorname{pf}(\widetilde{T}))$ & $R=\bar{R}$ \\
\hline$f=0$ & $(\operatorname{pf}(X))$ & $\frac{R}{(\operatorname{pf}(X))}$ & $\begin{array}{c}\text { no ideal } \\
\text { defined }\end{array}$ & $(\operatorname{pf}(X))$ & 0 \\
$g$ even & & $\frac{R}{I_{1}(Y)}$ & $R$ & $\left(y_{1}\right)$ & $\frac{R}{\left(y_{1}\right)}$ \\
\hline$g=1$ & $I_{1}(Y)$ & & & \\
\hline$f \geq 1$ & & & &
\end{tabular}

TABLE 4.14.a

Remarks 4.14.

(a) The information of Table 4.14.a has been collected for future convenience.

(b) Theorem 7.3 (c), together with Proposition 4.13 (c) and the right hand column in Table 4.14.a, shows that, if $\rho$ is a generic almost alternating map, then $H_{0}\left(\mathfrak{D}^{q}\right)$ is an $\bar{R}$-module for all integers $q$.

(c) In Theorem 7.3 we show that if $\rho$ is a generic almost alternating map, then the surjections of (d.iv) are actually isomorphisms. The situation $f=0$ and $g$ even is not treated by (d) because in this case, if $\rho$ is sufficiently general, then $M$ is an $\bar{R}$-module of rank two. (See Proposition 5.7.) For example, if $g=2$, then it is obvious that $M=\bar{R} \oplus \bar{R}$.

(d) In Theorem 9.13 we show that if $\rho$ is a generic almost alternating map, then it is possible to choose the ideal $\widetilde{J}$ so that the surjection in (e) is actually an isomorphism. Furthermore, if $R$ is a normal domain, then the $\bar{R}$-ideals $\widetilde{J} / J$ and $I^{q} \bar{R}$ are divisorial for $q \geq 1$, and $\left[H_{0}\left(\mathfrak{D}^{q}\right)\right]=q[I \bar{R}]$ in $C \ell(\bar{R})$ for all $q \geq-1$.

Proof of Proposition 4.13.

(a) We see, from the exact sequence

$$
Q_{0} \stackrel{d}{\rightarrow} K_{00} \longrightarrow H_{0}\left(\mathfrak{D}^{0}\right) \longrightarrow 0,
$$


that $H_{0}\left(\mathfrak{D}^{0}\right)$ is the cokernel of

$$
\sum_{r} \bigwedge^{g} G^{*} \otimes \bigwedge^{r} \stackrel{\sum \theta^{((g+r) / 2)}}{\longrightarrow} R
$$

where $r$ varies over all integers with $g+r$ even and $2-g \leq r \leq g$. The indicated inequalities actually put no restriction on $r$ because $g$ is at least one by hypotheses and the rank of $\theta$ is at most $2 g$ by Observation 3.17. The conclusion follows from Remark 4.11 (b).

(b) If $q \geq 1$, then the sequence

$$
K_{q-11} \longrightarrow K_{q 0} \longrightarrow H_{0}\left(\mathfrak{D}^{q}\right) \longrightarrow 0
$$

is exact. It is easy to see that this sequence is the same as

$$
\begin{array}{ll}
\frac{E^{*}}{\bigwedge^{g} G^{*} \otimes \bigwedge^{1-g} F} \stackrel{\rho}{\longrightarrow} G \longrightarrow H_{0}\left(\mathfrak{D}^{1}\right) \longrightarrow 0 & \text { if } q=1, \text { and } \\
\frac{S_{q-1} G \otimes \bigwedge^{1} E^{*}}{\eta S_{g-2} G} \longrightarrow \frac{\partial}{\longrightarrow} G \longrightarrow H_{0}\left(\mathfrak{D}^{q}\right) \longrightarrow 0 & \text { if } q>1,
\end{array}
$$

where $\partial$ is the Koszul algebra map associated to $\rho: E^{*} \rightarrow G$. The assertion is now obvious.

(c) In light of (a) and (b), it suffices to show that $J$ is contained in the annihilator of coker $\rho$. Take a basis for $G$ as described in Remark 2.2 (c). Let $g+r=2 p$, $x_{i}=\gamma_{i}^{*}$ for $1 \leq i \leq g$, and let $x_{g+1}, \ldots, x_{g+r}$ be arbitrary elements of $F$. Then, $\alpha=\theta^{(p)}\left(x_{1} \wedge \ldots \wedge x_{2 p}\right)$ is a typical element of $J$. We show that $\alpha \gamma_{1} \in \operatorname{im} \rho$. Let $x$ be the element

$$
\sum_{i=2}^{2 p}(-1)^{i+1} \theta^{(p-1)}\left(x_{2} \wedge \ldots \wedge \widehat{x_{i}} \wedge \ldots \wedge x_{2 p}\right) x_{i}
$$

of $E^{*}$. Use $(2.22)$ to see that $\rho(x)$ is equal to

$$
\sum_{i=2}^{2 p}(-1)^{i} \sum_{j=1}^{g}\left\langle\theta\left(\gamma_{j}^{*}\right), x_{i}\right\rangle \theta^{(p-1)}\left(x_{2} \wedge \ldots \wedge \widehat{x}_{i} \wedge \ldots \wedge x_{2 p}\right) \gamma_{j}
$$

Interchange the order of summation and apply (1.20) to see that

$$
\rho(x)=\sum_{j=1}^{g} \theta^{(p)}\left(\gamma_{j}^{*} \wedge x_{2} \wedge \ldots \wedge x_{2 p}\right) \gamma_{j}=\alpha \gamma_{1}
$$

(d.i) We first find an $R$-module surjection $M \rightarrow(I / A)$. The $R$-module $M$ is presented by

$$
R^{e} \stackrel{\left[\begin{array}{ll}
X & Y
\end{array}\right]}{\longrightarrow} R^{g} \longrightarrow M \longrightarrow 0
$$


consequently, it suffices to find an $R$-module surjection $\tau: R^{g} \rightarrow(I / A)$ so that

$$
R^{e} \stackrel{[X \quad Y}{\longrightarrow} R^{g} \stackrel{\tau}{\longrightarrow} I / A \longrightarrow 0
$$

is a complex. It is worthwhile to have uniform notation for the ideal $I$; so let $\widetilde{g}$ be the odd integer

$$
\widetilde{g}= \begin{cases}g & \text { if } g \text { is odd } \\ g+1 & \text { if } g \text { is even }\end{cases}
$$

$\widetilde{X}$ be the $\widetilde{g} \times \widetilde{g}$ alternating matrix

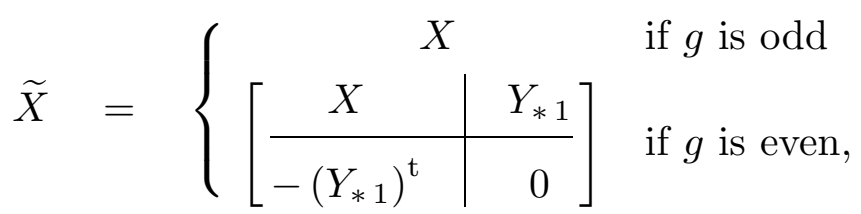

and $\widetilde{\mathbf{x}}$ be the $1 \times \widetilde{g}$ matrix $\left[\widetilde{X}_{\langle 1\rangle}, \ldots, \widetilde{X}_{\langle\widetilde{g}\rangle}\right]$ where $\widetilde{X}_{\langle j\rangle}$ is equal to $(-1)^{j+1}$ times the pfaffian of the matrix obtained by deleting row and column $j$ from $\widetilde{X}$. The ideal $I$ is generated by the entries of $\widetilde{\mathbf{x}}$; furthermore, it is well-known, and easy to see using (1.18) or (1.20), that

$$
0 \longrightarrow R \stackrel{(\widetilde{\mathbf{x}})^{\mathrm{t}}}{\longrightarrow} R^{\widetilde{g}} \stackrel{\widetilde{X}}{\longrightarrow} R^{\widetilde{g}} \stackrel{\widetilde{\mathbf{x}}}{\longrightarrow} I \longrightarrow 0
$$

is a complex. Let $u$ be the $1 \times g$ matrix

$$
u=\left[\tilde{X}_{\langle 1\rangle}, \ldots, \tilde{X}_{\langle g\rangle}\right]
$$

with entries from $R$. (If $g$ is odd, then $u=\widetilde{\mathbf{x}}$. If $g$ is even, then $u$ is one entry shorter than $\widetilde{\mathbf{x}}$.) Let $\tau: R^{g} \rightarrow(I / A)$ be the map induced by $u$. We claim that $\tau$ is a surjection which makes (4.15) be a complex. If $g$ is odd, then $\tau$ is clearly surjective. If $g$ is even, then

$$
\widetilde{X}_{\langle g+1\rangle}=\operatorname{pf}(X) \in A
$$

consequently, the map $\tau$ is surjective in all cases. Line (4.17), together with the fact that (4.16) is a complex, guarantees that every entry of $u X$ is in $A$. Observe that

$$
u Y= \begin{cases}{\left[a_{1}, \ldots, a_{f}\right]} & \text { if } g \text { is odd } \\ {\left[0, a_{2}, \ldots, a_{f}\right]} & \text { if } g \text { is even }\end{cases}
$$

where the $a$ 's are the generators of $A$ described in Definition 4.10. Thus, (4.15) is a complex and $\tau: M \rightarrow(I / A)$ is an $R$-module surjection. We proved in (c) that $M$ is an $\bar{R}$-module; consequently, $\tau$ is necessarily an $\bar{R}$-module surjection.

(d.ii) If grade $I \geq 3$, then Buchsbaum and Eisenbud [14, Theorem 2.1] have proved that (4.16) is exact. It follows readily, using (4.17) and (4.18), that (4.15) is exact. 
(d.iii) In (d.i) we proved that $I / A$ is an $(R / J)$-module. It follows that $I J \subseteq A$. It is clear from Definition 4.10 that $A \subseteq(I \cap J)$.

(d.iv) Combine (b), (d.i), and (d.iii) in order to obtain:

$$
H_{0}\left(\mathfrak{D}^{q}\right)=S_{q}(M) \rightarrow S_{q}(I / A) \rightarrow S_{q}\left(\frac{I}{I \cap J}\right)=S_{q}\left(\frac{I+J}{J}\right) \rightarrow \frac{I^{q}+J}{J}=I^{q} \bar{R} .
$$

(e) Every element $\gamma$ of $G$ gives rise to an $R$-ideal $\widetilde{J}=\widetilde{J}_{\gamma}$ which satisfies assertion (c). When we write $\gamma$ in terms of the basis $\gamma_{1}, \ldots, \gamma_{g}$ for $G$ we obtain $\gamma=\sum y_{i} \gamma_{i}$ for some $y_{i}$ in $R$. Let $\widetilde{T}$ be the alternating matrix

$$
\widetilde{T}=\left[\begin{array}{c|c|c} 
& y_{1} & \\
y_{2} & \\
\vdots & y_{g} & \\
-y_{1}-y_{2} \cdots-y_{g} & \multicolumn{2}{|c}{0} \\
\hline-Y^{\mathrm{t}} & &
\end{array}\right]
$$

which is obtained by "enlarging the $Y$ portion" of the matrix $T$ in (4.9). Define $\widetilde{J}_{\gamma}=\widetilde{J}$ to be the ideal of $R$ which is generated by the pfaffians of the principal submatrices of $\widetilde{T}$ which contain $X$.

We produce a surjection $H_{0}\left(\mathfrak{D}^{-1}\right) \rightarrow \widetilde{J} / J$ by considering a new almost alternating map

$$
\widetilde{\rho}=\left[\begin{array}{ll}
\xi \widetilde{\psi}
\end{array}\right]: \widetilde{E}^{*}=G^{*} \oplus \widetilde{F} \rightarrow G
$$

which is analogous to the almost alternating map $\rho: E^{*}=G^{*} \oplus F \rightarrow G$ of (2.3). Let $R \phi$ be a free $R$-module of rank one, let $\widetilde{F}=R \phi \oplus F$, and let $\widetilde{\psi}: \widetilde{F} \rightarrow G$ be defined by $\widetilde{\psi}(\phi)=\gamma$ and $\left.\widetilde{\psi}\right|_{F}=\psi$. Observe that the ideal $J(\widetilde{\rho})$ of Definition 4.10 formed using $\widetilde{\rho}$ is precisely equal to $\widetilde{J}$. Consider the complex

$$
\widetilde{L}_{0 g+2} \stackrel{\widetilde{d}}{\longrightarrow} \widetilde{Q}_{0} \stackrel{\widetilde{d}}{\longrightarrow} \widetilde{J} \rightarrow 0
$$

which is formed using $\widetilde{\rho}$. Observe that

$$
\widetilde{Q}_{0}=\left(Q_{-1} \otimes R \phi\right) \oplus Q_{0}, \quad \widetilde{L}_{0 g+2}=\left(L_{0 g+1} \otimes R \phi\right) \oplus L_{0 g+2}
$$

and that the diagram

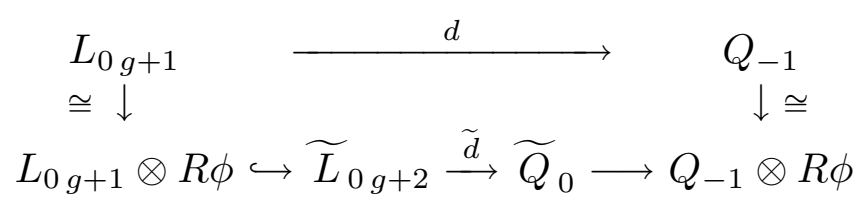


commutes. Since $\left.\widetilde{d}\right|_{Q_{0}}$ maps into $J$ we conclude that

$$
L_{0} g+1 \stackrel{d}{\rightarrow} Q_{-1} \rightarrow \widetilde{J} / J
$$

is a complex, where the second map is the composition

$$
Q_{-1} \stackrel{\cong}{\longrightarrow} Q_{-1} \otimes R \phi \hookrightarrow \widetilde{Q}_{0} \stackrel{\widetilde{d}}{\longrightarrow} \widetilde{J} \longrightarrow \widetilde{J} / J
$$

There is a connection between the complexes $\left\{\mathfrak{D}^{q}\right\}=\left\{\mathfrak{D}^{q}(\rho)\right\}$ constructed using a free module $F$ of rank $f$ and the complexes $\left\{\mathfrak{D}^{\prime q}\right\}=\left\{\mathfrak{D}^{q}\left(\rho^{\prime}\right)\right\}$ constructed using a free module $F^{\prime}$ of rank $f-1$. We have already exploited this connection, to some extent, in our proof of Proposition 4.13 (e). The short exact sequence of complexes of Proposition 4.23 is one of the main tools in our proof, in section 7 , that the complex $\mathfrak{D}^{q}$ is acyclic when $\rho$ is generic and $q \geq-1$.

Adopt the data of (2.3). Suppose that $f \geq 1$ and that $F=F^{\prime} \oplus R \phi$ where $F^{\prime}$ and $R \phi$ are free submodules of $F$ of rank $f-1$ and 1 , respectively. Let $\left(E^{\prime}\right)^{*}=G^{*} \oplus F^{\prime}$, and let $\rho^{\prime}$ be the composition

$$
\left(E^{\prime}\right)^{*} \stackrel{\text { incl }}{\longrightarrow} E^{*} \stackrel{\rho}{\longrightarrow} G .
$$

From $\left\{\mathfrak{D}^{q}\right\}$ and $J$ using the almost alternating map $\rho: E^{*} \rightarrow G$, and form $\left\{\mathfrak{D}^{q}\right\}$ and $J^{\prime}$ using the almost alternating map $\rho^{\prime}: E^{\prime *} \rightarrow G$. Let $M=\operatorname{coker} \rho$ and let $M^{\prime}=\operatorname{coker} \rho^{\prime}$.

The symmetric algebra $S_{\bullet}(M)$ is the polynomial ring $S_{\bullet}(G)$ modulo the ideal generated by the image of $\rho: E^{*} \rightarrow G$ in $G=S_{1}(G)$. We may view $\rho(\phi) \in G$ as an element of $S_{\bullet} G$. It is clear that

$$
S_{\bullet}(M)=\frac{S_{\bullet}(G)}{(\operatorname{im} \rho) S_{\bullet}(G)}=\frac{S_{\bullet}(G)}{\left(\operatorname{im} \rho^{\prime}+\rho(\phi)\right) S_{\bullet}(G)}=\frac{S_{\bullet}\left(M^{\prime}\right)}{(\rho(\phi)) S_{\bullet}\left(M^{\prime}\right)}
$$

In particular,

$$
S_{q-1}\left(M^{\prime}\right) \stackrel{\rho(\phi)}{\longrightarrow} S_{q}\left(M^{\prime}\right) \longrightarrow S_{q}(M) \longrightarrow 0
$$

is an exact sequence for all integers $q>0$.

We may apply Proposition 4.13 (e) to the complexes $\left(\mathfrak{D}^{\prime}\right)^{-1}$, and the element $\gamma=\rho(\phi)$. Observe that $\left(J^{\prime}\right)^{\sim}$ is precisely equal to $J$; and therefore, there is a surjection

$$
H_{0}\left(\mathfrak{D}^{\prime-1}\right) \rightarrow J / J^{\prime}
$$

induced by

$$
Q_{-1}^{\prime} \hookrightarrow Q_{-1}^{\prime} \otimes R \phi \hookrightarrow Q_{0} \stackrel{d}{\rightarrow} J \longrightarrow J / J^{\prime}
$$


Proposition 4.23. If $\rho$ and $\rho^{\prime}$ are defined as above, then for every integer $q$ there is a short exact sequence of complexes

$$
0 \longrightarrow \mathfrak{D}^{\prime q} \longrightarrow \mathfrak{D}^{q} \longrightarrow\left(\mathfrak{D}^{\prime q-1}\right)[-1] \longrightarrow 0 .
$$

In particular, there is a long exact sequence of homology

$$
\cdots \rightarrow H_{i+1}\left(\mathfrak{D}^{q}\right) \rightarrow H_{i}\left(\mathfrak{D}^{q-1}\right) \rightarrow H_{i}\left(\mathfrak{D}^{\prime q}\right) \rightarrow H_{i}\left(\mathfrak{D}^{q}\right) \rightarrow H_{i-1}\left(\mathfrak{D}^{q}\right) \rightarrow \cdots .
$$

Furthermore, the sequences

$$
H_{1}\left(\mathfrak{D}^{\prime q}\right) \longrightarrow H_{1}\left(\mathfrak{D}^{q}\right) \longrightarrow S_{q-1}\left(M^{\prime}\right) \stackrel{\rho(\phi)}{\longrightarrow} S_{q}\left(M^{\prime}\right) \longrightarrow S_{q}(M) \longrightarrow 0,
$$

for $q>0$, and

$$
H_{1}\left(\mathfrak{D}^{\prime 0}\right) \rightarrow H_{1}\left(\mathfrak{D}^{0}\right) \rightarrow H_{0}\left(\mathfrak{D}^{\prime-1}\right) \rightarrow J / J^{\prime} \rightarrow 0
$$

are exact where the last map is described in (4.22) and $S_{0}\left(M^{\prime}\right)$ means $R / J^{\prime}$.

Proof. For integers $i$ and $j$, form $Q_{i}, K_{i j}$, and $L_{i j}$ using the almost alternating map $\rho$ and form $Q_{i}^{\prime}, K_{i j}^{\prime}$, and $L_{i j}^{\prime}$ using the almost alternating map $\rho^{\prime}$. The decomposition $\bigwedge^{a} F=\bigwedge^{a} F^{\prime} \oplus\left(\bigwedge^{a-1} F^{\prime} \otimes R \phi\right)$ yields

$$
\begin{gathered}
Q_{i}=Q_{i}^{\prime} \oplus\left(Q_{i-1}^{\prime} \otimes R \phi\right), \quad K_{i j}=K_{i j}^{\prime} \oplus\left(K_{i j-1}^{\prime} \otimes R \phi\right), \quad \text { and } \\
L_{i j}=L_{i j}^{\prime} \oplus\left(L_{i j-1}^{\prime} \otimes R \phi\right) .
\end{gathered}
$$

Recall that

$$
\mathfrak{D}_{j}^{q}= \begin{cases}K_{q-j j} & \text { if } j \leq q \\ Q_{q} & \text { if } j=q+1 \\ L_{j-q-2 g+j} & \text { if } q+2 \leq j .\end{cases}
$$

It is now clear that $\mathfrak{D}_{j}^{q}=\mathfrak{D}_{j}^{q} \oplus\left(\mathfrak{D}_{j-1}^{q-1} \otimes R \phi\right)$ for all integers $j$ and $q$. These decompositions yield short exact sequences of modules:

$$
0 \rightarrow \mathfrak{D}_{j}^{\prime q} \rightarrow \mathfrak{D}_{j}^{q} \rightarrow \mathfrak{D}_{j-1}^{q-1} \rightarrow 0
$$

which fit together to form (4.24). The corresponding long exact sequence of homology is given in (4.25). In particular, if $i=0$ and $q>0$, then

$$
H_{1}\left(\mathfrak{D}^{q}\right) \rightarrow H_{1}\left(\mathfrak{D}^{q}\right) \rightarrow H_{0}\left(\mathfrak{D}^{q-1}\right) \stackrel{\delta}{\rightarrow} H_{0}\left(\mathfrak{D}^{\prime q}\right) \rightarrow H_{0}\left(\mathfrak{D}^{q}\right) \rightarrow H_{-1}\left(\mathfrak{D}^{q-1}\right)=0
$$

is an exact sequence (where $\delta$ is the connecting homomorphism). Proposition 4.13 (b) yields that $H_{0}\left(\mathfrak{D}^{\prime q-1}\right)=S_{q-1}\left(M^{\prime}\right), \quad H_{0}\left(\mathfrak{D}^{\prime q}\right)=S_{q}\left(M^{\prime}\right)$, and $H_{0}\left(\mathfrak{D}^{q}\right)=S_{q}(M)$. One may evaluate the connecting homomorphism $\delta$ in order to see that the right hand side of (4.27) is isomorphic to the exact sequence (4.21). 
Finally, if $i=q=0$, then (4.25) becomes the exact sequence

$$
H_{1}\left(\mathfrak{D}^{\prime 0}\right) \rightarrow H_{1}\left(\mathfrak{D}^{0}\right) \rightarrow H_{0}\left(\mathfrak{D}^{\prime-1}\right) \stackrel{\delta}{\rightarrow} H_{0}\left(\mathfrak{D}^{\prime 0}\right) \rightarrow H_{0}\left(\mathfrak{D}^{0}\right) \rightarrow H_{-1}\left(\mathfrak{D}^{\prime-1}\right)=0 .
$$

We learn from Proposition 4.13 (a) that $H_{0}\left(\mathfrak{D}^{\prime 0}\right)=R / J^{\prime}$ and $H_{0}\left(\mathfrak{D}^{0}\right)=R / J$. It is easy to see that the map $H_{0}\left(\mathfrak{D}^{\prime 0}\right) \rightarrow H_{0}\left(\mathfrak{D}^{0}\right)$ is precisely the natural quotient map. Thus, we have an exact sequence

$$
H_{1}\left(\mathfrak{D}^{\prime 0}\right) \rightarrow H_{1}\left(\mathfrak{D}^{0}\right) \rightarrow H_{0}\left(\mathfrak{D}^{\prime-1}\right) \stackrel{\delta}{\rightarrow} J / J^{\prime} \rightarrow 0
$$

The proof is completed by verifying that the connecting homomorphism $\delta$ is the same as the map of (4.22).

We conclude this section by discussing the degrees of the maps in the complexes $\left\{\mathfrak{D}^{q}\right\}$. When we view Figure 4.4 in terms of degrees, it resembles a boa constrictor eating a mouse. All of the maps are linear, except those involving $Q_{q}$, where there is a big bulge.

Observation 4.28. Form the complexes $\left\{\mathfrak{D}^{q}\right\}$ using generic data (as described in Remark 2.2(e)). If $q \geq 0$, then $\mathfrak{D}_{j}^{q}$ is equal to

$$
\begin{cases}R(-j)^{b_{q j}} & \text { if } j \leq q \\
\bigoplus_{r} R(-(g+r+q) / 2)^{\left(\begin{array}{l}
f \\
r
\end{array}\right)} & \text { if } j=q+1 \\
R(-(g-1+j))^{b_{q j}} & \text { if } q+2 \leq j\end{cases}
$$

where $r$ varies over all integers which satisfy the conditions of (2.16). In particular, if $0 \leq q \leq f-2$, then

$$
\mathfrak{D}_{f}^{q}=R(-(e-1))^{b_{q f}} \quad \text { where } \quad b_{q f}=\left(\begin{array}{c}
e-3-q \\
g-1
\end{array}\right) .
$$

Note. A formula for the Betti numbers $b_{q j}$ can be obtained by combining (4.26), Proposition 2.41, (2.24), and (2.39).

Proof. It suffices to consider $L_{0 g+2+q} \rightarrow Q_{q} \rightarrow K_{0 q}$. The map

$$
\bigwedge^{r} F \hookrightarrow Q_{q} \rightarrow K_{0 q}=R(-q)^{b_{q q}}
$$

has degree $(g+r-q) / 2$; and consequently, is a homogeneous map of degree zero:

$$
R(-(g+r+q) / 2)^{\left(\begin{array}{l}
f \\
r
\end{array}\right)} \rightarrow R(-q)^{b_{q q}} .
$$

The map $\mathfrak{D}_{q+2}^{q}=L_{0 g+2+q} \rightarrow Q_{q} \rightarrow \bigwedge^{r} F$ has degree $(g+2+q-r) / 2$; and consequently is a homogeneous map of degree zero:

$$
R(-(g+q+1))^{b_{q q+2}} \rightarrow R(-(g+r+q) / 2)^{\left(\begin{array}{l}
f \\
r
\end{array}\right) .}
$$




\section{Section 5. Grade CAlCulations.}

Ultimately, we prove that the complexes $\mathfrak{D}^{q}(\rho)$ are acyclic, for $q \geq-1$, provided the ideals $I_{t}(\rho)$ have sufficient grade. We have named the exact requirement "weak minor condition one" $\left(\mathrm{WMC}_{1}\right)$. (See Definitions 5.8 and 5.9.) In Observation 5.13 we show that every generic almost alternating map satisfies $\mathrm{WMC}_{1}$. In Propositions 5.14, 5.16, and 5.19 we give lower bounds for the grade of other relevant ideals in the generic situation. These results are essential for our proofs of the main theorems. The key step in all of these calculations is Lemma 5.2 which shows how to replace a given almost alternating map, one of whose entries is a unit, with an almost alternating map of smaller shape. The following hypotheses are used throughout the entire section:

$\rho=\left[\begin{array}{ll}\xi & \psi\end{array}\right]: G^{*} \oplus F \rightarrow G$ is an almost alternating map of shape $(g, f)$ over a commutative noetherian ring $R$, the complexes $\mathfrak{D}^{q}=\mathfrak{D}^{q}(\rho)$ are defined in section 2 , and the ideal $J=J(\rho)$ is defined in (4.10).

Lemma 5.2. Adopt the notation of (5.1). Suppose that there are bases for $F$ and $G$ so that the matrix of $\rho$ with respect to these bases is

$$
\left[\begin{array}{cccc|cc}
X_{1} & Y_{1} & 0 & 0 & Y_{2} & 0 \\
-\left(Y_{1}\right)^{\mathrm{t}} & X_{2} & 0 & 0 & 0 & I_{t} \\
0 & 0 & 0 & I_{s} & Y_{3} & Y_{4} \\
0 & 0 & -I_{s} & 0 & Y_{5} & Y_{6}
\end{array}\right]
$$

for some integers $s$ and $t$ with $2 s+t \leq g-1$, some alternating matrices $X_{1}$ and $\mathrm{X}_{2}$ and some arbitrary matrices $Y_{i}$ for $1 \leq i \leq 6$. If $\rho^{\prime}$ is the map which is represented by the matrix $\left[X_{1} \mid Y_{1} Y_{2}\right]$, then $\rho^{\prime}$ is an almost alternating map of shape $(g-2 s-t, f)$ and the following statements hold.

(a) The ideals $J(\rho)$ and $J\left(\rho^{\prime}\right)$ are equal.

(b) If $q \geq 0$, then the modules $H_{0}\left(\mathfrak{D}^{q}(\rho)\right)$ and $H_{0}\left(\mathfrak{D}^{q}\left(\rho^{\prime}\right)\right)$ are isomorphic.

(c) If $1 \leq k \leq g-2 s-t$, then $I_{k}\left(\rho^{\prime}\right)=I_{2 s+t+k}(\rho)$.

(d) If $k$ is an even integer with $2 \leq k \leq g-2 s$, then the ideal $\operatorname{Pf}_{2 s+k}(\xi)$ is equal to

$$
\operatorname{Pf}_{k}\left[\begin{array}{cc}
X_{1} & Y_{1} \\
-\left(Y_{1}\right)^{\mathrm{t}} & X_{2}
\end{array}\right] \text {. }
$$

Proof. It is clear that $\rho^{\prime}$ is an almost alternating map of shape $(g-2 s-t, f)$, and that $\rho^{\prime}$ satisfies (c) and (d). Assertion (b) for $q>0$ follows from Proposition 4.13 because coker $\rho \cong \operatorname{coker} \rho^{\prime}$. We still must show that $J(\rho)$ and $J\left(\rho^{\prime}\right)$ are equal as ideals. (Recall from (4.10) that the ideal $J(\rho)$ is generated by certain pfaffians of the matrix $T$ of (4.9).) It suffices to assume that, either, $s=0$ and $t=1$, or else that, $s=1$ and $t=0$. In the first case, there is a one-to-one correspondence between the pfaffians of the principal submatrices of

$$
T=\left[\begin{array}{cc|cc}
X_{1} & Y_{1} & Y_{2} & 0 \\
-\left(Y_{1}\right)^{\mathrm{t}} & 0 & 0 & 1 \\
\hline-\left(Y_{2}\right)^{\mathrm{t}} & 0 & 0 & 0 \\
0 & -1 & 0 & 0
\end{array}\right]
$$

$$
\text { which contain } \quad\left[\begin{array}{cc}
X_{1} & Y_{1} \\
-\left(Y_{1}\right)^{\mathrm{t}} & 0
\end{array}\right]
$$

and 
the pfaffians of the principal submatrices of

$$
T^{\prime}=\left[\begin{array}{c|cc}
X_{1} & Y_{1} & Y_{2} \\
\hline-\left(Y_{1}\right)^{\mathrm{t}} & 0 & 0 \\
-\left(Y_{2}\right)^{\mathrm{t}} & 0 & 0
\end{array}\right]
$$

which contain $X_{1}$. Notice, for example, that

$$
\operatorname{pf}\left[\begin{array}{cc|cc}
X_{1} & Y_{1} & Z & 0 \\
-\left(Y_{1}\right)^{\mathrm{t}} & 0 & 0 & 1 \\
\hline-Z^{\mathrm{t}} & 0 & 0 & 0 \\
0 & -1 & 0 & 0
\end{array}\right]= \pm \mathrm{pf}\left[\begin{array}{cc}
X_{1} & Z \\
-Z^{\mathrm{t}} & 0
\end{array}\right]
$$

for any submatrix $Z$ of $Y_{2}$ of the appropriate shape. In the second case,

$$
T=\left[\begin{array}{ccc|c}
X_{1} & 0 & 0 & Y_{2} \\
0 & 0 & 1 & Y_{3} \\
0 & -1 & 0 & Y_{5} \\
\hline-\left(Y_{2}\right)^{\mathrm{t}} & -\left(Y_{3}\right)^{\mathrm{t}} & -\left(Y_{5}\right)^{\mathrm{t}} & 0
\end{array}\right] \quad \text { and } \quad T^{\prime}=\left[\begin{array}{c|c}
X_{1} & Y_{2} \\
\hline-\left(Y_{2}\right)^{\mathrm{t}} & 0
\end{array}\right]
$$

It is obvious that $J(\rho)=J\left(\rho^{\prime}\right)$.

Lemma 5.4. If the ring $R$ of (5.1) is local and $I_{\ell}(\rho)=R$ for some positive integer $\ell$, then, either, $I_{g}(\rho)=J=R$, or else, there is an almost alternating map $\rho^{\prime}$ of shape $\left(g^{\prime}, f\right)$ such that:

(a) $1 \leq g^{\prime} \leq g-\ell$

(b) the ideals $J(\rho)$ and $J\left(\rho^{\prime}\right)$ are equal.

(c) $H_{0}\left(\mathfrak{D}^{q}(\rho)\right) \cong H_{0}\left(\mathfrak{D}^{q}\left(\rho^{\prime}\right)\right)$ for all $q \geq 0$, and

(d) $I_{k}\left(\rho^{\prime}\right)=I_{g-g^{\prime}+k}(\rho)$ for all $k$ with $1 \leq k \leq g^{\prime}$.

Proof. We first prove that there are bases for $G$ and $F$ such that the matrix of $\rho$ with respect to these bases has the form of (5.3) where $\ell \leq(2 s+t)$. Let $u$ be the largest integer with $I_{u}(\xi)=R$. Since $\xi$ is an alternating map over a local ring, we know that $u$ is even and we write $u=2 s$. The module $G$ may be decomposed into a direct sum of free modules $G_{1} \oplus G_{2} \oplus G_{2}^{*}$ (with $\operatorname{rank} G_{2}=s$ ) in such a way that the $\operatorname{map} \xi: G_{1}^{*} \oplus G_{2}^{*} \oplus G_{2} \rightarrow G_{1} \oplus G_{2} \oplus G_{2}^{*}$ is

$$
\left[\begin{array}{ccc}
\xi_{1} & 0 & 0 \\
0 & 0 & I_{s} \\
0 & -I_{s} & 0
\end{array}\right]
$$

for some alternating map $\xi_{1}: G_{1}^{*} \rightarrow G_{1}$. (At this point, we have chosen a basis for $G_{2}$, and the corresponding dual basis for $G_{2}^{*}$. We have not yet picked a basis for $G_{1}$ or $F$.) If $2 s \geq \ell$, then the proof is complete. If $2 s<\ell$, then let $t$ be the difference $\ell-2 s$ and let $\psi_{1}$ be $\psi$ followed by the projection $G=G_{1} \oplus G_{2} \oplus G_{2}^{*} \rightarrow G_{1}$. The 
hypothesis guarantees that $I_{t}\left(\psi_{1}\right)=R$. In other words, we may choose bases for $G_{1}$ and $F$ so that the matrix for $\psi_{1}$ has the form

$$
\left[\begin{array}{cc}
Y_{2} & 0 \\
0 & I_{t}
\end{array}\right]
$$

When $\rho$ is written with respect to the bases we have chosen, then, either, $2 s+t=g$, or else $\rho$ has the form of (5.3). In the second case the proof is completed by applying Lemma 5.2. It remains to show that if $I_{g}(\rho)=R$, then $J$ is also equal to $R$. This calculation is rather easy because we know that

$$
\rho=\left[\begin{array}{ccc|cc}
X_{2} & 0 & 0 & 0 & I_{t} \\
0 & 0 & I_{s} & Y_{3} & Y_{4} \\
0 & -I_{s} & 0 & Y_{5} & Y_{6}
\end{array}\right]
$$

and every entry of $X_{2}$ is in the maximal ideal of $R$. It follows that the matrix $T$ of (4.9) is

$$
T=\left[\begin{array}{ccc|cc}
X_{2} & 0 & 0 & 0 & I_{t} \\
0 & 0 & I_{s} & Y_{3} & Y_{4} \\
0 & -I_{s} & 0 & Y_{5} & Y_{6} \\
\hline 0 & -\left(Y_{3}\right)^{\mathrm{t}} & -\left(Y_{5}\right)^{\mathrm{t}} & 0 & 0 \\
-I_{t} & -\left(Y_{4}\right)^{\mathrm{t}} & -\left(Y_{6}\right)^{\mathrm{t}} & 0 & 0
\end{array}\right]
$$

The pfaffian of the principal $2(s+t) \times 2(s+t)$ submatrix

$$
\left[\begin{array}{ccc|c}
X_{2} & 0 & 0 & I_{t} \\
0 & 0 & I_{s} & Y_{4} \\
0 & -I_{s} & 0 & Y_{6} \\
\hline-I_{t} & -\left(Y_{4}\right)^{\mathrm{t}} & -\left(Y_{6}\right)^{\mathrm{t}} & 0
\end{array}\right]
$$

is a unit in $R$; hence, $J=I_{g}(\rho)=R$.

If $P$ is a prime ideal of the ring $R$, then $\kappa(P)$ is the residue field $(R / P)_{P}$ of the local ring $R_{P}$.

Corollary 5.5. The ideals $I_{g}(\rho)$ and $J$ of (5.1) have the same radical.

Proof. Let $P$ be any prime ideal of $R$. If $I_{g}(\rho)$ is not contained in $P$, then rank $\rho \otimes$ $\kappa(P)=g$, and Lemma 5.4 shows that $J(\rho \otimes \kappa(P))=\kappa(P)$. It follows that $J(\rho)$ is also not contained in $P$. If $I_{g}(\rho) \subseteq P$, then $\operatorname{rank} \rho \otimes \kappa(P)<g$, and there are bases such that $\rho \otimes \kappa(P)$ has the form of (5.3) with every entry of $X_{1}, X_{2}, Y_{1}$, and $Y_{2}$ equal to zero. It follows that $J(\rho \otimes \kappa(P))=J\left(\rho^{\prime}\right)$ for $\rho^{\prime}=\left[\begin{array}{lll}0 & 0 & 0\end{array}\right]$. It is clear that $J\left(\rho^{\prime}\right)=0$. We conclude that $J(\rho)$ is also contained in $P$.

Let $\rho=\left[\begin{array}{ll}X & Y\end{array}\right]$ be a generic almost alternating matrix. The next result shows how to apply Lemma 5.2 to $\rho$ in order to obtain a smaller generic almost alternating matrix $\rho^{\prime}$ over a larger base ring. 
Lemma 5.6. Let $R_{0}$ be a commutative noetherian ring; $\rho=\left[\begin{array}{ll}X & Y\end{array}\right]$ be a generic almost alternating map of shape $(g, f)$ over the polynomial ring $R=R_{0}[X, Y]$, and let $P$ be a prime ideal of $R$. Suppose that $\operatorname{Pf}_{2 s}(X)$ is not contained in $P$ for some even integer $2 s$ with $2 \leq 2 s \leq g-1$. Then, there are elements $z_{1}, \ldots, z_{n}, \Delta$ of $R$ and there is an almost alternating matrix $\rho^{\prime}$ of shape $(g-2 s, f)$ with entries in $R\left[\Delta^{-1}\right]$ so that

(a) $\rho^{\prime}$ is obtained from $\rho \otimes_{R} R\left[\Delta^{-1}\right]$ by using the technique of Lemma 5.2;

(b) $z_{1}, \ldots, z_{n}$ are algebraically independent over $R_{0}$;

(c) $\Delta \in R_{0}\left[z_{1}, \ldots, z_{n}\right]$ but $\Delta \notin P$;

(d) the (distinct non-zero) entries of $\rho^{\prime}$ are algebraically independent over

$$
S=R_{0}\left[z_{1}, \ldots, z_{n}, \Delta^{-1}\right] ;
$$

(e) $S\left[\rho^{\prime}\right]=R\left[\Delta^{-1}\right]$; and

(f) $(R / J)\left[\Delta^{-1}\right]=R\left[\Delta^{-1}\right] / J\left(\rho^{\prime}\right)$.

Furthermore, if $R_{0}$ is a domain, then the element $\Delta$ of $R_{0}\left[z_{1}, \ldots, z_{n}\right]$ can be factored into a product of prime elements.

Note. There are two variations in the hypothesis which lead to essentially the same conclusion. If $I_{t}(Y)$ is not contained in $P$ for some $t$ with $1 \leq t \leq g-1$, then the same conclusion holds for a map $\rho^{\prime}$ of shape $(g-t, f)$. If $I_{g}(\rho) \subseteq P$, but $I_{\ell}(\rho)$ is not contained in $P$ for some $\ell$ with $1 \leq \ell \leq g-1$, then the same conclusion holds for a map $\rho^{\prime}$ of shape $\left(g^{\prime}, f\right)$ for some $g^{\prime}$ with $1 \leq g^{\prime} \leq g-\ell$.

Proof. We may prove this result by induction on $s$; and therefore, it suffices to

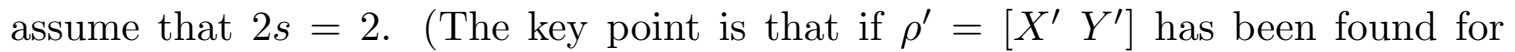
$2 s=2$, then, according to Lemma $5.2(\mathrm{~d}), \mathrm{Pf}_{k}\left(X^{\prime}\right)=\mathrm{Pf}_{k+2}(X)$ for all even $k$.) We may assume, without loss of generality, that $\Delta=x_{g-1 g} \notin P$. Let $z_{1}, \ldots, z_{n}$ be the set of elements

$$
\left\{\begin{array}{ll}
x_{i g-1} & 1 \leq i \leq g-2 \\
x_{i g} & 1 \leq i \leq g-1 \\
y_{i j} & g-1 \leq i \leq g
\end{array} \text { and } \quad 1 \leq j \leq f\right.
$$

of $R$. It is obvious that (b) and (c) hold. After a change of bases, $\rho \otimes_{R} R\left[\Delta^{-1}\right]$ may be put in the form of (5.3); that is,

$$
\rho \otimes_{R} R\left[\Delta^{-1}\right]=\left[\begin{array}{ccc|c}
X_{1} & 0 & 0 & Y_{2} \\
0 & 0 & 1 & Y_{3} \\
0 & -1 & 0 & Y_{5}
\end{array}\right] .
$$

Let $\rho^{\prime}=\left[\begin{array}{ll}X_{1} & Y_{2}\end{array}\right]$. Observe that (a) also holds. It is not difficult to see that (d) and (e) hold. Assertion (f) is implied by (a). Finally, if $R_{0}$ is a domain, then it is obvious that the variable $\Delta$ is a prime element of $R_{0}\left[z_{1}, \ldots, z_{n}\right]$.

The proof of the stated result is complete. A word about the two variations is in order. If $I_{t}(Y)$ is not contained in $P$, then there is no need to do induction: simply take $\Delta$ to be one of the $t \times t$ minors of $Y$ which is not in $P$. If $I_{\ell}(\rho)$ is not contained in $P$, then prove the result in two steps: first apply the stated result to $\operatorname{Pf}_{2 s}(X)$ where $s$ is the largest integer with $\operatorname{Pf}_{2 s}(X)$ not contained in $P$, then apply the first variation.

The next result can be used to calculate the ranks of the $(R / J)$-modules $H_{0}\left(\mathfrak{D}^{q}\right)$. 
Proposition 5.7. Adopt the notation of (5.1). Let $q \geq 0$ be an integer and let $P$ be a prime ideal of $R$ with $J \subseteq P$.

(a) Suppose that, either, $f>0$, or else, that $f=0$ and $g$ is odd. If

$$
f+1 \leq \text { grade } I_{g-1}(\rho) \quad \text { and } \quad \text { grade } P \leq f,
$$

then $H_{0}\left(\mathfrak{D}^{q}\right)_{P} \cong H_{0}\left(\mathfrak{D}^{0}\right)_{P}$ and $J_{P}$ is generated by $f$ elements.

(b) Suppose $f=0$ and $g$ is even. If grade $I_{g-2}(\rho) \geq f+2$ and grade $P \leq f+1$, then $H_{0}\left(\mathfrak{D}^{1}\right)_{P} \cong H_{0}\left(\mathfrak{D}^{0}\right)_{P} \oplus H_{0}\left(\mathfrak{D}^{0}\right)_{P}$.

Note. In our typical application of this result we will already know that $J$ is a perfect ideal with

$$
\text { grade } J= \begin{cases}f+1 & \text { if } f=0 \text { and } g \text { is even } \\ f & \text { in all other cases. }\end{cases}
$$

In this case, the statement of Proposition 5.7 becomes:

If grade $I_{t}(\rho) \geq 1+$ grade $J$ for $t=f+g-1$-grade $J$, then the $(R / J)$-module $H_{0}\left(\mathfrak{D}^{q}\right)$ has rank equal to

$$
\left\{\begin{array}{ll}
1 & \text { if } q \geq 0 \text { and grade } J=f \\
2 & \text { if } q=1 \text { and grade } J=f+1
\end{array} .\right.
$$

Proof.

(a) Lemma 5.4 shows that $H_{0}\left(\mathfrak{D}^{i}\left(\rho \otimes R_{P}\right)\right)=H_{0}\left(\mathfrak{D}^{i}\left(\rho^{\prime}\right)\right)$ for some almost alternating map $\rho^{\prime}$ of shape $(1, f)$ for all $i \geq 0$. So, in particular, $J_{P}=J\left(\rho^{\prime}\right)$. Example 4.8 shows that $H_{0}\left(\mathfrak{D}^{q}\left(\rho^{\prime}\right)\right)=H_{0}\left(\mathfrak{D}^{0}\left(\rho^{\prime}\right)\right)$ and that $J\left(\rho^{\prime}\right)$ is generated by $f$ elements.

(b) The argument is the same as the argument in (a), except this time $\rho^{\prime}$ has shape $(2,0)$. If

$$
\rho^{\prime}=\left[\begin{array}{rr}
0 & x \\
-x & 0
\end{array}\right]
$$

then $J\left(\rho^{\prime}\right)=(x)$ and

$$
\operatorname{coker} \rho^{\prime}=\frac{R_{P}}{(x)} \oplus \frac{R_{P}}{(x)} \text {. }
$$

We now give conditions which ensure that the complexes $\mathfrak{D}^{q}(\rho)$ are acyclic. For $f>0$ our conditions are expressed in terms of the grade of the ideals $I_{t}(\rho)$; however, if $f=0$, then $\rho$ is an alternating map and it is more natural to express our conditions in terms of the lower order pfaffians of $\rho$.

Definition 5.8. If $\rho$ is an almost alternating map of shape $(g, f)$ and $r$ is a fixed integer with $1 \leq r \leq g$, then

(a) $\rho$ satisfies the Weak Minor Condition $\mathrm{WMC}_{r}$ if grade $I_{g}(\rho) \geq f$ and grade $I_{t}(\rho) \geq f+g-t$ for all $t$ with $r \leq t \leq g-1$;

(b) $\rho$ satisfies the Strong Minor Condition $\operatorname{SMC}_{r}$ if grade $I_{g}(\rho) \geq f$ and grade $I_{t}(\rho) \geq f+g-t+1$ for all $t$ with $r \leq t \leq g-1$. 
Note. The minor conditions $\mathrm{WMC}_{g}$ and $\mathrm{SMC}_{g}$ are exactly the same; and the map $\rho$ satisfies these conditions provided grade $I_{g}(\rho) \geq f$. The minor conditions have been defined so that

$$
\mathrm{MC}_{1} \Longrightarrow \mathrm{MC}_{2} \Longrightarrow \cdots \Longrightarrow \mathrm{MC}_{g} \quad \text { and } \quad \mathrm{SMC}_{r} \Longrightarrow \mathrm{WMC}_{r} \text {. }
$$

Definition 5.9. Let $G$ be a free $R$-module of rank $g$ and let $r$ be a fixed integer with $1 \leq r \leq g-1$. If $\xi: G^{*} \rightarrow G$ is an alternating map, then

(a) $\xi$ satisfies the Weak Pfaffian Condition $\mathrm{WPC}_{r}$ if grade $\mathrm{Pf}_{t}(\xi) \geq g-t+1$ for all even $t$ with $r+1 \leq t \leq g$;

(b) $\xi$ satisfies the Strong Pfaffian Condition $\mathrm{SPC}_{r}$ if grade $\mathrm{Pf}_{t}(\xi) \geq g-t+2$ for all even $t$ with $r+1 \leq t \leq g-1$, and grade $\operatorname{Pf}_{g}(\xi) \geq 1$ if $g$ is even.

Note. The pfaffian conditions $\mathrm{WPC}_{g-1}$ and $\mathrm{SPC}_{g-1}$ are exactly the same; and the map $\xi$ satisfies these conditions provided, either $g$ is odd, or else, $g$ is even and the ideal $\operatorname{Pf}_{g}(\xi)$ contains a regular element of $R$. The pfaffian conditions have been defined so that

$$
\mathrm{PC}_{1} \Longrightarrow \mathrm{PC}_{2} \Longrightarrow \cdots \Longrightarrow \mathrm{PC}_{g-1} \quad \text { and } \quad \mathrm{SPC}_{r} \Longrightarrow \mathrm{WPC}_{r}
$$

If $f=0$, then we are able to relate the minor conditions and the pfaffian conditions because of the following well known result.

Observation 5.10. Let $G$ be a free $R$-module of rank $g$. If $\xi: G^{*} \rightarrow G$ is an alternating map and $t$ is an even integer with $2 \leq t \leq g$, then the $R$-ideals

$$
I_{t-1}(\xi), \quad I_{t}(\xi), \quad \text { and } \quad \operatorname{Pf}_{t}(\xi)
$$

all have the same radical.

Proof. If $P$ is a prime ideal of $R$, then the alternating map $\xi \otimes \kappa(P)$ has even rank; consequently, $P$ contains one of the listed ideals if and only if it contains all of them.

Corollary 5.11. If $\rho$ is an almost alternating map of shape $(g, 0)$ and $r$ is a fixed integer with $1 \leq r \leq g-1$, then

(a) $\rho$ satisfies $\mathrm{WMC}_{r}$ if and only if $\rho$ satisfies $\mathrm{WPC}_{r}$ and $\operatorname{grade} \operatorname{Pf}_{r}(\rho) \geq g-r$ if $r$ is even;

(b) $\rho$ satisfies $\mathrm{SMC}_{r}$ if and only if $\rho$ satisfies $\operatorname{SPC}_{r}$ and grade $\operatorname{Pf}_{g}(\rho) \geq 2$ if $g$ is even and grade $\operatorname{Pf}_{r}(\rho) \geq g-r+1$ if $r$ is even.

The next result, which is used in the proof of Corollary 8.9, has essentially the same proof as Observation 5.10; so, we include it here.

\section{Observation 5.12.}

(a) Adopt the notation of (5.1) with $f=1$. Let $\theta$ be the corresponding alternating map of (2.3). If $t$ is an even integer, then the $R$-ideals $\operatorname{Pf}_{t}(\theta)$ and $I_{t-1}(\rho)$ have the same radical.

(b) Adopt the notation of Definition 4.10 with either $g$ odd or $f$ positive. Then grade $I_{g-1}(\rho) \geq \operatorname{grade}(I+J)$. 
Proof.

(a) Let $P$ be a prime ideal of $R$. If $2 s$ denotes the rank of $(\xi \otimes \kappa(P))$, then both of the following equations hold for one fixed $r$ equal to 0 or 1 :

$$
\operatorname{rank}(\theta \otimes \kappa(P))=2 s+2 r \quad \text { and } \quad \operatorname{rank}(\rho \otimes \kappa(P))=2 s+r .
$$

Observe that $\operatorname{Pf}_{t}(\theta) \subseteq P$ if and only if $2 s+2 r<t$; and $I_{t-1}(\rho) \subseteq P$ if and only if $2 s+r<t-1$. Since $t$ is even and $r$ is equal to 0 or 1 , we quickly see that $2 s+2 r<t$ if and only if $2 s+r<t-1$.

(b) We show that $(I+J) \subseteq \operatorname{rad}\left(I_{g-1}(\rho)\right)$. It is clear from Corollary 5.5 that

$$
J \subseteq \operatorname{rad}\left(I_{g}(\rho)\right) \subseteq \operatorname{rad}\left(I_{g-1}(\rho)\right)
$$

If $g$ is odd, then $I$ is defined to be $\operatorname{Pf}_{g-1}(X)$; hence Observation 5.10 shows that

$$
I \subseteq \operatorname{rad}\left(I_{g-1}(X)\right) \subseteq \operatorname{rad}\left(I_{g-1}(\rho)\right)
$$

If $g$ is even, then $I$ is defined to be

$$
\operatorname{Pf}_{g}\left[\begin{array}{c|c}
X & Y_{* 1} \\
\hline-\left(Y_{* 1}\right)^{\mathrm{t}} & 0
\end{array}\right]
$$

and (a) shows that $I \subseteq \operatorname{rad}\left(I_{g-1}\left[X Y_{* 1}\right]\right) \subseteq \operatorname{rad}\left(I_{g-1}(\rho)\right)$.

We conclude this section by giving lower bounds for various relevant ideals in the generic situation.

Observation 5.13. If $\rho$ is a generic almost alternating map of shape $(g, f)$ and $t$ is an integer with $1 \leq t \leq g$, then

$$
\text { grade } I_{t}(\rho) \geq \frac{(g-t+1)(g-t)}{2}+(g-t+1) f
$$

furthermore, the following statements hold.

(a) If, either, $f \geq 1$, or else, $f=0$ and $g$ is odd, then $\rho$ satisfies $\mathrm{SMC}_{1}$.

(b) The map $\rho$ satisfies $\mathrm{WMC}_{1}$ for all $f$ and $g$.

(c) If $f=0$ and $g \geq 2$, then $\rho$ satisfies $\mathrm{SPC}_{1}$.

Note. If $f=0$ and $g$ is even, then it is impossible for $\rho$ to satisfy $\mathrm{SMC}_{r}$ for any $r$ with $1 \leq r \leq g-1$ because $I_{g-1}(\rho)$ and $I_{g}(\rho)$ have the same grade and this grade is at most one.

Proof. Let $g(t)$ represent the right hand side of the above inequality. We prove the inequality by induction on $t$. The assertion is obvious if $t=1$, because $g(1)$ is the number of distinct non-zero entries in the generic almost alternating matrix which represents $\rho$. Suppose that $P$ is a prime ideal of $R$ with $I_{t}(\rho) \subseteq P$. It suffices to show that grade $P R_{P} \geq g(t)$ because for any ideal $I$ in a $\operatorname{ring} R$, the grade of $I$ is equal to

$$
\min \left\{\operatorname{grade} P R_{P} \mid P \in \operatorname{Spec} R \quad \text { and } \quad I \subseteq P\right\}
$$


If $I_{t-1}(\rho) \subseteq P$, then we know, by induction, that grade $P \geq g(t-1) \geq g(t)$. If $I_{t-1}(\rho)$ is not contained in $P$, then we apply Lemma 5.6 in order to obtain a generic almost alternating map $\rho^{\prime}$ of shape $(g-t+1, f)$ with $I_{t}\left(\rho \otimes R_{P}\right)=I_{1}\left(\rho^{\prime}\right)$. It is clear that grade $I_{1}\left(\rho^{\prime}\right)=g(t)$. We conclude that grade $I_{t}(\rho) \geq g(t)$ for all $t$.

Observe that

$$
g(t)=\left\{\begin{array}{ll}
=f & \text { if } t=g \\
=2 f+1 & \text { if } t=g-1 \\
\geq f+g-t+1 & \text { if } 1 \leq t \leq g-2
\end{array}= \begin{cases}=f & \text { if } t=g \\
\geq f+g-t+1 & \text { if } t=g-1 \text { and } f \geq 1 \\
=f+g-t & \text { if } t=g-1 \text { and } f=0 \\
\geq f+g-t+1 & \text { if } 1 \leq t \leq g-2 .\end{cases}\right.
$$

It is clear that $\rho$ satisfies $\mathrm{WMC}_{1}$ for all $f$ and $g$, and that $\rho$ satisfies $\mathrm{SMC}_{1}$ if $f \geq 1$. If $f=0$ and $g$ is odd, then, according to Observation 5.10, the ideals $I_{g-1}(\rho)$ and $I_{g-2}(\rho)$ have the same grade; and so $\rho$ satisfies $\mathrm{SMC}_{1}$. Assertion (c) follows immediately from (1.24).

Proposition 5.14. Let $\rho=[X Y]$ be a generic alternating map of shape $(g, f)$.

(a) If $s$ is an integer with $2 \leq 2 s \leq g$, then

$$
\operatorname{grade}\left(J+\operatorname{Pf}_{2 s}(X)\right) \geq\left(\begin{array}{c}
g-2 s+2 \\
2
\end{array}\right)+f-g+2 s-1 .
$$

(b) Assume that, either, $g$ is odd, or else, $f$ is positive. If $I=I(\rho)$ is the ideal defined in (4.10), then grade $(I+J) \geq f+1$.

Note. Assertion (a) guarantees that

$$
\left\{\begin{array}{l}
\operatorname{grade}\left(J+\operatorname{Pf}_{g-1}(X)\right) \geq f+1 \quad \text { if } g \text { is odd, } \\
\operatorname{grade}\left(J+\operatorname{Pf}_{g-2}(X)\right) \geq f+3 \quad \text { if } g \text { is even, and } \\
\operatorname{grade}\left(J+\operatorname{Pf}_{g-3}(X)\right) \geq f+6 \quad \text { if } g \geq 3 \text { is odd }
\end{array}\right.
$$

Proof.

(a) The proof is very similar to the proof of Observation 5.13. Let $g(s)$ represent the right hand side of the inequality. Recall the grade of $\operatorname{Pf}_{2 s}(X)$ from (1.24). Observe that the inequality is valid if $f-g+2 s-1 \leq 0$; henceforth, we assume that

$$
g-2 s+2 \leq f
$$

Let $P$ be a prime ideal which contains $J+\operatorname{Pf}_{2 s}(X)$. Since $g(s-1) \geq g(s)$, we may assume that $P$ does not contain $\mathrm{Pf}_{2 s-2}(X)$. According to Lemma 5.6, there are bases so that the matrix of $\rho \otimes R_{P}$ is equal to

$$
\left[\begin{array}{ccc|c}
X_{1} & 0 & 0 & Y_{2} \\
0 & 0 & I_{s-1} & Y_{3} \\
0 & -I_{s-1} & 0 & Y_{5}
\end{array}\right]
$$


where $\left[X_{1} Y_{2}\right]$ is a generic almost alternating map. The prime ideal $P$ contains $\mathrm{Pf}_{2 s}(X)$; consequently, $\mathrm{Pf}_{2}\left(X_{1}\right) \subseteq P R_{P}$. The ideal $P$ also contains $J$; and therefore, by Corollary 5.5,

$$
I_{g-2 s+2}\left(Y_{2}\right) \subseteq I_{g}\left(\rho \otimes R_{P}\right) \subseteq P R_{P} .
$$

The generic alternating matrix $X_{1}$ has size $(g-2 s+2) \times(g-2 s+2)$; the generic matrix $Y_{2}$ has size $(g-2 s+2) \times f$ and $g-2 s+2 \leq f$ by (5.15). Apply (1.24) and (1.23) in order see that

$$
\text { grade } P R_{P} \geq\left(\begin{array}{c}
g-2 s+2 \\
2
\end{array}\right)+f-(g-2 s+2)+1
$$

(b) In light of (a), we need only consider the case where $g \geq 2$ is even and $f \geq 1$. As before we suppose that $P$ is a prime ideal of $R$ with grade $P R_{P} \leq f$ and $(I+J) \subseteq P$. Observe that $I_{g}\left(\left[\begin{array}{ll}X & Y^{\prime}\end{array}\right]\right) \subseteq P$ where $Y^{\prime}$ is the matrix formed by deleting the first column of $Y$. Observation 5.13 shows that

$$
\operatorname{grade} I_{g}\left(\left[X Y^{\prime}\right]\right) \geq f-1 \text {. }
$$

It follows that $y_{r 1} \notin P$ for some $r$; because if $y_{11}, y_{21}, \ldots, y_{g 1}$ are all also in $P$, then

$$
\text { grade } P \geq g+f-1 \geq f+1 \text {. }
$$

Suppose that $y_{g 1} \notin P$. Again we apply the technique of Lemma 5.6 and pick bases for $G$ and $F$ (without changing $\phi_{1}$ ) so that the matrix of $\rho \otimes R_{P}$ is

$$
\left[\begin{array}{cc|cc}
X_{1} & Y_{1} & 0 & Y_{2} \\
-\left(Y_{1}\right)^{\mathrm{t}} & 0 & 1 & 0
\end{array}\right]
$$

Observe that

$$
I_{P}=\operatorname{Pf}_{g}\left[\begin{array}{cc|c}
X_{1} & Y_{1} & 0 \\
-\left(Y_{1}\right)^{\mathrm{t}} & 0 & 1 \\
\hline 0 & -1 & 0
\end{array}\right]=\operatorname{Pf}_{g-2}\left(X_{1}\right)
$$

furthermore, $J\left(\rho \otimes R_{P}\right)=J\left(\rho^{\prime}\right)$ where $\rho^{\prime}$ is the generic alternating map

$$
\left[\begin{array}{llll}
X_{1} & \mid & Y_{1} & Y_{2}
\end{array}\right]
$$

The shape of $\rho^{\prime}$ is $(g-1, f)$ and $g-1$ is odd. The proof is completed by appealing to part (a).

Proposition 5.16. Let $\rho=\left[\begin{array}{l}X \\ Y\end{array}\right]$ be a generic almost alternating matrix of shape $(g, f)$. Assume that $f \geq 1$ if $g$ is odd and that $f \geq 2$ if $g$ is even. Let $Y_{* j}$ represent the $j^{\text {th }}$ column of $Y$; $Y^{\prime}$ be the matrix obtained by removing the last column from the matrix $Y ; \rho^{\prime}$ be the generic almost alternating map $\left[X Y^{\prime}\right]$ of shape $(g, f-1)$; and $J^{\prime}$ be the ideal $J\left(\rho^{\prime}\right)$. If $z$ is the pfaffian of the alternating matrix

$$
\widetilde{\widetilde{X}}=\left\{\begin{array}{c|cc}
{\left[\begin{array}{c|c}
X & Y_{* f} \\
\hline-\left(Y_{* f}\right)^{\mathrm{t}} & 0
\end{array}\right]} & \text { if } g \text { is odd } \\
{\left[\begin{array}{c|cc}
X & Y_{* 1} & Y_{* f} \\
\hline-\left(Y_{* 1}\right)^{\mathrm{t}} & 0 & 0 \\
-\left(Y_{* f}\right)^{\mathrm{t}} & 0 & 0
\end{array}\right] \quad \text { if } g \text { is even, }}
\end{array}\right.
$$


then grade $\left(J^{\prime}, z\right) \geq f$.

Proof. Suppose that $P$ is a prime ideal of $R$ with grade $P R_{P} \leq f-1$ and $\left(J^{\prime}, z\right) \subseteq P$. We first consider the case when $g$ is odd. Proposition 5.14 shows that $\operatorname{Pf}_{g-1}(X)$ is not contained in $P$. By applying the technique of Lemma 5.6 we may choose a basis for $G$ (without changing the basis of $F$ ) so that

$$
\rho \otimes R_{P}=\left[\begin{array}{ccc|c}
0 & 0 & 0 & Y_{2} \\
0 & 0 & I_{s} & Y_{3} \\
0 & -I_{s} & 0 & Y_{5}
\end{array}\right] .
$$

The $1 \times f$ matrix $Y_{2}$ is generic and $I_{1}\left(Y_{2}\right) \subseteq P R_{P}$. (The first $f-1$ elements of $Y_{2}$ are in $P R_{P}$ because $J^{\prime} \subseteq P$. The last element of $Y_{2}$ is in $P R_{P}$ because $z \in P$.) The assumption that grade $P R_{P} \leq f-1$ has been contradicted and the proof is complete when $g$ is odd.

If $g$ is even, then Proposition 5.14 shows that $\operatorname{Pf}_{g-2}(X)$ is not contained in $P$. There is a basis for $G$ such that

$$
\rho \otimes R_{P}=\left[\begin{array}{cccc|c}
0 & x & 0 & 0 & y_{11} \cdots y_{1 f} \\
-x & 0 & 0 & 0 & y_{21} \cdots y_{2 f} \\
0 & 0 & 0 & I_{s} & Y_{3} \\
0 & 0 & -I_{s} & 0 & Y_{5}
\end{array}\right]
$$

Observe that

$$
\left(I_{2}\left[\begin{array}{lll}
y_{11} & \cdots & y_{1 f-1} \\
y_{21} & \cdots & y_{2 f-1}
\end{array}\right], x\right) \subseteq J_{P}^{\prime}
$$

and that the ideal $(z, x)_{P}$ is generated by $x$ together with

$$
\operatorname{det}\left[\begin{array}{ll}
y_{11} & y_{1 f} \\
y_{21} & y_{2 f}
\end{array}\right] \text {. }
$$

Lemma 5.18 shows that grade $P R_{P} \geq f$. This contradiction completes the proof.

Lemma 5.18. If $R_{0}$ is a commutative noetherian ring; $f \geq 2$ is an integer; and

$$
Y=\left[\begin{array}{lll}
y_{11} & \cdots & y_{1 f} \\
y_{21} & \cdots & y_{2 f}
\end{array}\right]
$$

is a matrix of indeterminates, then the ideal

$$
A=\left(I_{2}\left[\begin{array}{lll}
y_{11} & \cdots & y_{1 f-1} \\
y_{21} & \cdots & y_{2 f-1}
\end{array}\right], \quad \operatorname{det}\left[\begin{array}{ll}
y_{11} & y_{1 f} \\
y_{21} & y_{2 f}
\end{array}\right]\right)
$$

of the ring $R=R_{0}[Y]$ has grade $f-1$.

Proof. The ideal $A$ is a contained in $I_{2}(Y)$; thus, grade $A \leq f-1$ by (1.23). The result is obvious if $f=2$; so we assume that $f \geq 3$. Let $P$ be a prime ideal of $R$ which contains $A$. If $y_{11}$ and $y_{21}$ are both in $P$, then grade $P \geq f-1$ because either $f-1=2$ or $P$ also contains $I_{2}\left(Y^{\prime}\right)$ where $Y^{\prime}$ is the generic $2 \times(f-2)$ matrix obtained by deleting columns 1 and $f$ from $Y$. If $y_{21} \notin P$, then it is easy to see that the elements

$$
\left\{y_{21} y_{1 i}-y_{2 i} y_{11} \mid 2 \leq i \leq f\right\}
$$

form a regular sequence in $P R_{P}$. 
Proposition 5.19. Let $\rho=\left[\begin{array}{ll}X & Y\end{array}\right]$ be a generic almost alternating matrix of shape $(g, f)$ with $g \geq 2$. Let $X^{\prime}$ be the matrix obtained by deleting the last row and column from $X$; and let $Y^{\prime}$ be the matrix obtained by deleting the last row of $Y$. If $\rho^{\prime}$ is the generic almost alternating matrix $\left[\begin{array}{ll}X^{\prime} & Y^{\prime}\end{array}\right]$ of shape $(g-1, f)$, then $(f+1) \leq \operatorname{grade}\left(J(\rho)+J\left(\rho^{\prime}\right)\right)$.

Proof. If $g$ is even and $f=0$, then $J\left(\rho^{\prime}\right)=0$, but $J(\rho)$ has grade $f+1$. Henceforth, we assume that either, $g$ is odd, or else, $f \geq 1$. If $g-1$ is odd, then let

$$
z=\operatorname{pf}\left[\begin{array}{c|c}
X^{\prime} & x_{1 g} \\
& x_{2 g} \\
& \vdots \\
& x_{g-1 g} \\
\hline-x_{1 g}-x_{2 g} \cdots-x_{g-1 g} & 0
\end{array}\right] .
$$

If $g-1$ is even, then let

$$
z=\operatorname{pf}\left[\begin{array}{c|cc} 
& x_{1 g} & y_{11} \\
X^{\prime} & x_{2 g} & y_{21} \\
& \vdots & \vdots \\
& x_{g-1 g} & y_{g-11} \\
\hline-x_{1 g}-x_{2 g} \cdots-x_{g-1 g} & 0 & y_{g 1} \\
-y_{11}-y_{21} \cdots-y_{g-11} & -y_{g 1} & 0
\end{array}\right]
$$

and let $z^{\prime}$ be $z$ with $y_{g 1}$ set equal to zero. It is clear that $z \in J(\rho)$. If $g-1$ is even, then it is also clear that $z=z^{\prime} \pm y_{g 1} \operatorname{pf}\left(X^{\prime}\right)$; thus, $z-z^{\prime} \in J(\rho)$. Proposition 5.16 shows that

$$
f+1 \leq \operatorname{grade}\left(J\left(\rho^{\prime}\right), z\right) \leq \operatorname{grade}\left(J(\rho)+J\left(\rho^{\prime}\right)\right) .
$$


Section 6. Acyclicity in the Case $f=0$.

Throughout this section

$f=0$ and $\rho$ is the alternating map $\xi$; the complexes $\mathfrak{D}^{q}$ are formed using the data of (2.3); and the ideal $J=\mathrm{Pf}_{g}(\xi)$ is defined in (4.10).

A picture of the complexes $\left\{\mathfrak{D}^{q}\right\}$ may be found in Figures 4.6 and 4.7. The conditions WPC and SPC are defined in (5.9). There are two main results in this section. The first is

Theorem 6.2. Adopt the notation of (6.1). Let $r$ and $q$ be fixed integers such that $1 \leq r \leq g-1,0 \leq q$ and $\ell\left(\mathfrak{D}^{q}\right) \leq g-r$. If $\xi$ satisfies $\mathrm{WPC}_{r}$, then $\mathfrak{D}^{q}$ is acyclic.

Note. The length, $\ell\left(\mathfrak{D}^{q}\right)$, of $\mathfrak{D}^{q}$ is given in Observation 4.3. Recall that $\ell\left(\mathfrak{D}^{q}\right) \leq$ $g-1$ for all $q$. Recall, also, that if the alternating map $\xi$ is generic, then it satisfies $\mathrm{WPC}_{1}$. (We say that a complex $\mathbb{F}$ is acyclic if $\mathbb{F}_{j}=0$ for $j<0$ and $H_{j}(\mathbb{F})=0$ for $j>0$. If $\mathbb{F}$ is an acyclic complex of projective modules, then $\mathbb{F}$ is a projection resolution of $H_{0}(\mathbb{F})$.)

The other main result is Corollary 6.25 , where we calculate depth $\left(R / I^{q}\right)$, for all $q \leq 1$, whenever $I$ is a sufficiently general grade three Gorenstein ideal in a local ring $R$. Our proof of Theorem 6.2 consists of two parts. We use the BuchsbaumEisenbud criterion to reduce to the case where $R$ is a field. Then, we prove the necessary result (Lemma 6.5) under that hypothesis.

Theorem 6.3. ([11]) Let $R$ be a commutative noetherian ring and let

$$
\mathbb{A}: \quad 0 \longrightarrow A_{n} \stackrel{a_{n}}{\longrightarrow} A_{n-1} \longrightarrow \cdots \longrightarrow A_{1} \stackrel{a_{1}}{\longrightarrow} A_{0}
$$

be a complex of free $R$-modules. Then $\mathbb{A}$ is acyclic if and only if

(a) $\operatorname{rank} a_{k+1}+\operatorname{rank} a_{k}=\operatorname{rank} A_{k}$, and

(b) grade $I\left(a_{k}\right) \geq k$

for $1 \leq k \leq n$.

The following piece of folklore is well-known.

Observation 6.4. Let $\mathbb{A}$ and $R$ be as in Theorem 6.3.

(a) If $(R, \mathfrak{m}, k)$ is a local ring and $\mathbb{A} \otimes k$ is acyclic, then $H_{0}(\mathbb{A})$ is a free $R-\operatorname{module}$ and $\mathbb{A}$ is acyclic.

(b) If $\mathbb{A} \otimes \kappa(P)$ is acyclic for all associated prime ideals $P$ of $R$, then $\mathbb{A}$ satisfies the rank condition of Theorem 6.3.

Proof.

(a) Every finite free complex $\mathbb{A}$ over a local ring $(R, k)$ decomposes into a direct sum $\mathbb{A}^{\prime} \oplus \mathbb{A}^{\prime \prime}$ of complexes, where $\mathbb{A}^{\prime}$ is split exact and $\mathbb{A}^{\prime \prime} \otimes k$ is complex with zero differential. The hypothesis guarantees that $\mathbb{A}^{\prime \prime}$ looks like $\cdots \longrightarrow 0 \longrightarrow 0 \longrightarrow A_{0}^{\prime \prime}$.

(b) Fix an integer $k$ with $1 \leq k \leq n$. We must show that rank $a_{k}$ is equal to $r$, where $r$ is defined by

$$
r=\operatorname{rank} A_{k}-\operatorname{rank} A_{k+1}+\operatorname{rank} A_{k+2}-\cdots+(-1)^{n-k} \operatorname{rank} A_{n} .
$$


The hypothesis, together with (a) and Theorem 6.3, guarantees that rank $\left(a_{k}\right)_{P}=r$ for all $P \in$ Ass $R$. It is clear that

$$
\operatorname{rank} a_{k} \geq \operatorname{rank}\left(a_{k}\right)_{P}=r \text {. }
$$

Furthermore, if $t>r$, then $\left(I_{t}\left(a_{k}\right)\right)_{P}=I_{t}\left(\left(a_{k}\right)_{P}\right)=0$ for all $P \in$ Ass $R$; hence, $I_{t}\left(a_{k}\right)=0$ and $r \geq \operatorname{rank} a_{k}$.

Lemma 6.5. Suppose that $f=0$ and $R=k$ is a field. If the complexes $\mathfrak{D}^{q}$ are formed using the data of (2.3), then $H_{j}\left(\mathfrak{D}^{q}\right)=0$ for all $q \geq 0$ and for all $j \geq g-\operatorname{rank} \xi$.

Assume, for the time being, that Lemma 6.5 has been established.

Proof of Theorem 6.2. Let $\ell$ be the length of $\mathfrak{D}^{q}$. A quick look at Observation 4.3 (f) and $(\mathrm{g})$, or Figures 4.6 and 4.7 , shows that $g-\ell$ is odd; consequently, we may assume, without loss of generality, that

$$
2 \leq \ell \leq g-r \quad \text { if } g \text { is odd } \quad \text { and } \quad 1 \leq \ell \leq g-r \quad \text { if } g \text { is even. }
$$

Let $P$ be an associated prime of $R$ and let $t$ be the rank of $\xi$. Since $\xi$ satisfies $\mathrm{WPC}_{r}$, and $r$ satisfies the above constraints, we know that $t=g$ if $g$ is even, $t=g-1$ if $g$ is odd, and that $\operatorname{Pf}_{t}(\xi)$ is not contained in $P$. Apply Lemma 6.5 to $\xi \otimes \kappa(P)$ in order to see that $\mathfrak{D}^{q} \otimes \kappa(P)$ is acyclic. Observation 6.4 (b) shows that $\mathfrak{D}^{q}$ satisfies condition (a) of Theorem 6.3. Furthermore, the complex $\mathfrak{D}^{q} \otimes R_{Q}$ also satisfies condition (a) of Theorem 6.3 for all prime ideals $Q$ of $R$; consequently,

$$
\operatorname{rank} d_{j}=\operatorname{rank}\left(d_{j} \otimes R_{Q}\right) \quad \text { for all } i \text { and for all } Q
$$

Let $j$ be a fixed integer with $1 \leq j \leq \ell$. Define $t$ to be the largest even integer with $t \leq g-j+1$. Observe that

$$
r \leq g-\ell \leq g-j \leq t \leq g-j+1 \leq g \text { and } g-t \leq j \leq g-t+1
$$

Furthermore, we know that $r<t$ because $t$ is even and $g-\ell$ is odd. The map $\xi$ satisfies $\mathrm{WPC}_{r}$; consequently, $j \leq g-t+1 \leq \operatorname{grade~}_{t}(\xi)$.

Let $I_{j}$ be the $j^{\text {th }}$ Fitting ideal, $I\left(d_{j}\right)$, of the complex $\mathfrak{D}^{q}$. Let $P$ be a prime ideal of $R$ with $I_{j} \subseteq P$. We prove that $\operatorname{Pf}_{t}(\xi)$ is also contained in $P$; hence, $j \leq$ grade $P$. Let $s$ be the rank of $\xi \otimes \kappa(P)$. Suppose that $\operatorname{Pf}_{t}(\xi)$ is not contained in $P$. It follows that $s \geq t$. Let $\mathfrak{D}_{\text {trunc }}^{q}$ be the following truncation of the complex $\mathfrak{D}^{q}$ :

$$
0 \longrightarrow \mathfrak{D}_{\ell}^{q} \longrightarrow \cdots \longrightarrow \mathfrak{D}_{g-s-1}^{q}
$$

We see, from Lemma 6.5, that $\mathfrak{D}_{\text {trunc }}^{q} \otimes \kappa(P)$ is acyclic. It follows, from Observation 6.4 (a), that $\mathfrak{D}_{\text {trunc }}^{q} \otimes R_{P}$ is split exact. In particular, the Fitting ideal $I_{k}$ of $\mathfrak{D}^{q}$ is not contained in $P$ for any $k$ with $1 \leq k$ and $g-s \leq k$. (Recall, from (6.6), that $I_{k} R_{P}$ is the $k^{\text {th }}$ Fitting ideal of $\mathfrak{D}^{q} \otimes R_{P}$.) This is a contradiction because $1 \leq j$, $g-s \leq g-t \leq j$, and $I_{j} \subseteq P$. Thus, $\mathfrak{D}^{q}$ also satisfies condition (b) of Theorem 6.3, and is consequently acyclic. 
Proof of Lemma 6.5. In this proof $q$ always represents a non-negative integer. Let $t$ be the rank of $\xi$. The rank $t$ is necessarily even because $\xi$ is an alternating map over a field. It $t=0$, then the assertion holds since $\mathfrak{D}_{j}^{q}=0$ for all $j \geq g$. Henceforth, we assume that $t \geq 2$. Let $S$ be the symmetric algebra $S_{\bullet} G$. Observe that $S$ is a polynomial ring in $g$ variables over the base field $k$. As in (2.6), we let $\mathbb{K}$ be the Koszul algebra associated to the map $\xi: G^{*} \rightarrow G$. Recall, from (1.10), that $\mathbb{K}$ is "the usual Koszul complex"

$$
\bigwedge_{S}\left(S \otimes_{k} G^{*}\right)
$$

which is associated to the map $\Xi$ :

$$
S \otimes_{k} G^{*} \stackrel{1 \otimes \xi}{\longrightarrow} S \otimes_{k} G \stackrel{\text { mult }}{\longrightarrow} S
$$

from the free, rank $g, S$-module $S \otimes_{k} G^{*}$ to $S$. The image of $\Xi$ is an ideal in $S$ which is generated by $t$ linearly independent one forms. Thus, the image of $\Xi$ has grade $t$, and

$$
H_{j}(\mathbb{K})=0 \quad \text { for all } j \text { with } g-t<j .
$$

Exactly as in the proof of Proposition 2.17, the algebra $\mathbb{K}$ decomposes into a direct sum of graded strands $\mathbb{K}=\oplus \mathbb{K}^{q}$, where $\mathbb{K}^{q}$ is the complex

$$
\mathbb{K}^{q}: \quad 0 \rightarrow S_{0} G \otimes \bigwedge^{q} G^{*} \rightarrow \cdots \rightarrow S_{q-1} G \otimes \bigwedge^{1} G^{*} \rightarrow S_{q} G \otimes \bigwedge^{0} G^{*}
$$

(with $S_{q} G \otimes \bigwedge^{0} G^{*}$ in position zero). It follows, from (6.7) that

$$
H_{j}\left(\mathbb{K}^{q}\right)=0 \quad \text { whenever } 0 \leq q \text { and } g-t<j .
$$

It is necessary for us to define a further family of complexes $\left\{\widetilde{\mathbb{K}}^{q}\right\}$, which differs very little from $\left\{\mathbb{K}^{q}\right\}$. If $q \neq g$, then let $\widetilde{\mathbb{K}}^{q}$ be the same as $\mathbb{K}^{q}$. Define $\widetilde{\mathbb{K}}^{g}$ to be the following truncation of $\mathbb{K}^{g}$ :

$$
\widetilde{\mathbb{K}}_{j}^{g}= \begin{cases}\mathbb{K}_{j}^{g} & \text { if } j<g \\ 0 & \text { if } g \leq j\end{cases}
$$

Recall, from (6.8), that $H_{j}\left(\mathbb{K}^{g}\right)=0$ for $j>g-2$ (since $\left.2 \leq t\right)$. It follows that $H_{g-1}\left(\widetilde{\mathbb{K}}^{g}\right)$ is equal to $k$; consequently,

$$
H_{j}\left(\widetilde{\mathbb{K}}^{q}\right)= \begin{cases}k & \text { if } j=g-1 \text { and } q=g \\ 0 & \text { otherwise }\end{cases}
$$

for all integers $q$ and $j$ with $0 \leq q$ and $g-t<j$.

We also define truncated complexes $\left\{\widetilde{\mathfrak{D}}^{q}\right\}$ according to the rule

$$
\widetilde{\mathfrak{D}}_{j}^{q}= \begin{cases}\mathfrak{D}_{j}^{q} & \text { if } j \leq q \\ 0 & \text { if } q<j .\end{cases}
$$


Notice that $\mathfrak{D}_{j}^{q}=0$ for $q+1<j$ and

$$
\mathfrak{D}_{q+1}^{q}= \begin{cases}k & \text { if } 0 \leq q \leq g-2 \text { and } g+q \text { is even } \\ 0 & \text { otherwise. }\end{cases}
$$

Let $q$ be an index with $\mathfrak{D}_{q+1}^{q}$ not zero. The map

$$
k=\mathfrak{D}_{q+1}^{q} \rightarrow \mathfrak{D}_{q}^{q}
$$

involves the pfaffians of all of the principal submatrices of $\xi$ of size $g-q$. This map is zero if $t<g-q$ and this map is not zero if $g-q \leq t$. We draw two conclusions. First of all, if $0 \leq j \leq q$, then

$$
H_{j}\left(\widetilde{\mathfrak{D}}^{q}\right)= \begin{cases}H_{j}\left(\mathfrak{D}^{q}\right) \oplus k & \text { if } g-t \leq j=q \leq g-2 \text { and } g+q \text { is even } \\ H_{j}\left(\mathfrak{D}^{q}\right) & \text { otherwise. }\end{cases}
$$

Secondly, if $0 \leq q<j$ and $H_{j}\left(\mathfrak{D}^{q}\right) \neq 0$, then

$$
j=q+1 \leq g-t \quad \text { and } \quad q+g \text { is even. }
$$

Observe, that there is no further need for us to consider $H_{j}\left(\mathfrak{D}^{q}\right)$ with $0 \leq q<j$; because, if $g-t \leq j, 0 \leq q<j$, and $H_{j}\left(\mathfrak{D}^{q}\right) \neq 0$, then $g-t=j=q+1$, which is impossible since $t$ and $g+q$ are both even.

In Lemma 6.13 we prove that for each integer $q \geq 0$, there is a short exact sequence of complexes

$$
0 \rightarrow \widetilde{\mathfrak{D}}^{q}[-1] \rightarrow \widetilde{\mathbb{K}}^{q+2} \rightarrow \widetilde{\mathfrak{D}}^{q+2} \rightarrow 0
$$

The corresponding long exact sequence of homology yields an exact sequence

$$
H_{j+2}\left(\widetilde{\mathfrak{D}}^{q+2}\right) \rightarrow H_{j}\left(\widetilde{\mathfrak{D}}^{q}\right) \rightarrow H_{j+1}\left(\widetilde{\mathbb{K}}^{q+2}\right)
$$

for all integers $j$ and $q$ with $q \geq 0$. We apply (6.12) three times, always using (6.10) and (6.9) in order to interpret the homology of $\widetilde{\mathfrak{D}}$ and $\widetilde{\mathbb{K}}$.

Suppose that $g-t \leq j \leq q$ and that, either, $q \neq j$, or else that, $q=j$ but $g+q$ is odd. The exact sequence (6.12) yields

$$
H_{j+2}\left(\mathfrak{D}^{q+2}\right) \rightarrow H_{j}\left(\mathfrak{D}^{q}\right) \rightarrow 0 .
$$

If this process is iterated, then we obtain a series of surjections:

$$
H_{j+2 N}\left(\mathfrak{D}^{q+2 N}\right) \rightarrow \cdots \rightarrow H_{j+2}\left(\mathfrak{D}^{q+2}\right) \rightarrow H_{j}\left(\mathfrak{D}^{q}\right) .
$$

The left most module is zero when $j+2 N \geq g$, thus $H_{j}\left(\mathfrak{D}^{q}\right)$ is also zero.

If $j=q=g-2$, then (6.12) becomes the exact sequence

$$
0 \rightarrow H_{g-2}\left(\mathfrak{D}^{g-2}\right) \oplus k \rightarrow k .
$$

We conclude that $H_{g-2}\left(\mathfrak{D}^{g-2}\right)=0$. Finally, if $g-t \leq j=q \leq g-4$ and $g+q$ is even, then (6.12) becomes

$$
H_{q+2}\left(\mathfrak{D}^{q+2}\right) \oplus k \rightarrow H_{q}\left(\mathfrak{D}^{q}\right) \oplus k \rightarrow 0 .
$$

If this process is iterated, then we obtain a series of surjections:

$$
k=H_{g-2}\left(\mathfrak{D}^{g-2}\right) \oplus k \rightarrow \cdots \rightarrow H_{q+2}\left(\mathfrak{D}^{q+2}\right) \oplus k \rightarrow H_{q}\left(\mathfrak{D}^{q}\right) \oplus k,
$$

from which we conclude that $H_{q}\left(\mathfrak{D}^{q}\right)=0$. 
Lemma 6.13. For each integer $q \geq 0$, line (6.11) is a short exact sequence of complexes.

Proof. It is clear, from Proposition 2.17, that

$$
\mathbb{K}^{q}[-1] \stackrel{\eta}{\longrightarrow} \mathbb{K}^{q+2} \longrightarrow \widetilde{\mathfrak{D}}^{q+2} \longrightarrow 0
$$

is an exact sequence of complexes for all $q \geq 0$, except $q=g-2$. The sequence (6.14) looks like:

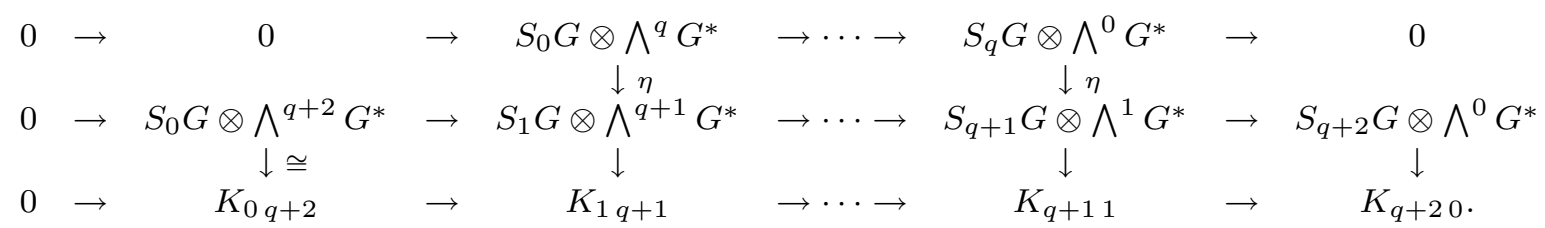

The module $K_{a b}$ is defined to be the indicated cokernel (provided $(a, b) \neq(0, g)$ ) and the differential in $\widetilde{\mathfrak{D}}^{q+2}$ is induced by the differential in $\mathbb{K}^{q+2}$. If $q=g-2$, then $K_{0 q+2}=K_{0 g}$ is zero, but $S_{0} G \otimes \bigwedge^{q+2} G^{*} \neq 0$. We defined the complex $\widetilde{\mathbb{K}}^{g}$ to be the truncation of $\mathbb{K}^{g}$ which makes

$$
\mathbb{K}^{q}[-1] \stackrel{\eta}{\longrightarrow} \widetilde{\mathbb{K}}^{q+2} \longrightarrow \widetilde{\mathfrak{D}}^{q+2} \longrightarrow 0
$$

be exact for $q=g-2$. There is no difference between (6.14) and (6.15) for $q \neq g-2$. Thus, (6.15) holds for all $q \geq 0$.

Let $\overline{\mathbb{K}}^{q}$ represent the complex

$$
0 \rightarrow \frac{S_{0} G \otimes \bigwedge^{q} G^{*}}{\operatorname{Ker} \eta} \rightarrow \cdots \rightarrow \frac{S_{q-1} G \otimes \bigwedge^{1} G^{*}}{\operatorname{Ker} \eta} \rightarrow \frac{S_{q} G \otimes \bigwedge^{1} G^{*}}{\operatorname{Ker} \eta} \rightarrow 0
$$

We see from (6.15) that

$$
0 \rightarrow \overline{\mathbb{K}}^{q}[-1] \stackrel{\eta}{\rightarrow} \widetilde{\mathbb{K}}^{q+2} \rightarrow \widetilde{\mathfrak{D}}^{q+2} \rightarrow 0
$$

is a short exact sequence of complexes. The proof is complete as soon as we prove that the complexes $\overline{\mathbb{K}}^{q}$ and $\widetilde{\mathfrak{D}}^{q}$ are equal. Observe that $\overline{\mathbb{K}}_{g}^{g}=0=\widetilde{\mathfrak{D}}_{g}^{g}$. It suffices to show that

$$
\operatorname{Ker}\left(\eta: S_{a} G \otimes \bigwedge^{b} G^{*} \rightarrow S_{a+1} G \otimes \bigwedge^{b+1} G^{*}\right)=\eta\left(S_{a-1} G \otimes \bigwedge^{b-1} G^{*}\right)
$$

for all pairs of integers $(a, b)$ except $(a, b)=(0, g)$. Fortunately, we have already made the calculation which is necessary to establish (6.16). Let $\mathbb{L}=\left(S \bullet G \otimes \bigwedge^{\bullet} G, \partial\right)$ be the Koszul algebra associated to the identity map on $G$. The graded strand

$$
\mathbb{L}^{i}: \quad 0 \rightarrow S_{0} G \otimes \bigwedge^{i} G \rightarrow S_{1} G \otimes \bigwedge^{i-1} G \rightarrow \cdots \rightarrow S_{i} G \otimes \bigwedge^{0} G
$$


of $\mathbb{L}$ is split exact for all $i>0$. Diagram (2.44) shows that the complex $\mathbb{L}^{i}$ is isomorphic to

$$
0 \rightarrow S_{0} G \otimes \bigwedge^{g-i} G^{*} \stackrel{\eta}{\rightarrow} S_{1} G \otimes \bigwedge^{g-i+1} G^{*} \stackrel{\eta}{\rightarrow} \cdots \stackrel{\eta}{\rightarrow} S_{i} G \otimes \bigwedge^{g} G^{*}
$$

It follows that

$$
S_{a-1} G \otimes \bigwedge^{b-1} G^{*} \stackrel{\eta}{\rightarrow} S_{a} G \otimes \bigwedge^{b} G^{*} \stackrel{\eta}{\rightarrow} S_{a+1} G \otimes \bigwedge^{b+1} G^{*}
$$

is an exact sequence for all pairs of integer $(\mathrm{a}, \mathrm{b})$ provided $b \neq a+g$.

In Theorem 6.17 we expand and reformulate Theorem 6.2. The most interesting addition is part $(\mathrm{d})$. The other parts have been added in order to facilitate comparison between the present result and the main theorem of the paper, which is Theorem 8.3.

Theorem 6.17. Adopt the notation of (6.1). If $I_{g}(\xi)=R$, then every complex $\mathfrak{D}^{q}$ is split exact. If $I_{g}(\xi)$ is a proper ideal of $R$ and $\xi$ satisfies $\mathrm{WPC}_{g-1}$, then the following statements hold.

(a) The ideal $J$ is perfect. If $g$ is odd, then $J$ is the zero ideal. If $g$ is even, then grade $J=1$.

(b) If $\mathfrak{D}^{q}$ has length zero, then $\mathfrak{D}^{q}$ is acyclic and $H_{0}\left(\mathfrak{D}^{q}\right)=R=R / J$.

$\left(\mathrm{b}^{\prime}\right)$ If $J$ has grade one, then

(i) the complexes $\mathfrak{D}^{0}$ and $\mathfrak{D}^{1}$ are acyclic,

(ii) the $R$-module $H_{0}\left(\mathfrak{D}^{1}\right)$ is perfect of projective dimension one, and

(iii) the $(R / J)$-module $H_{0}\left(\mathfrak{D}^{1}\right)$ is torsion-free.

(c) If $\xi$ satisfies the condition $\mathrm{WPC}_{r}$ for some integer $r$ with $1 \leq r \leq g-1$, and $q \geq-1$ is an integer with $\ell\left(\mathfrak{D}^{q}\right) \leq g-r$, then

(i) the complex $\mathfrak{D}^{q}$ is acyclic,

(ii) $H_{j}\left(\mathfrak{D}^{-2-q}\right)=\operatorname{Ext}_{R}^{-j}\left(H_{0}\left(\mathfrak{D}^{q}\right), R\right)$ for all integers $j$, and

(iii) $H_{j}\left(\mathfrak{D}^{-2-q}\right)=0$ for $j \geq 1$.

(d) If $\xi$ satisfies $\mathrm{SPC}_{r}$ for some integer $r$ with $1 \leq r \leq g-1$, and $q \geq-1$ is an integer with $\ell\left(\mathfrak{D}^{q}\right) \leq g-r$, then the following statements hold.

(i) The $(R / J)$-module $H_{0}\left(\mathfrak{D}^{q}\right)$ is torsion-free,

(ii) if $q \geq 1, g$ is odd, and $I$ is the ideal $\mathrm{Pf}_{g-1}(\xi)$ of $R$, then

$$
H_{0}\left(\mathfrak{D}^{q}\right) \cong S_{q}\left(I^{q}\right) \cong I^{q}
$$

(iii) if $g$ is even and grade $\operatorname{Pf}_{g-2}(\xi) \geq 2$, then the rank of the $(R / J)$-module $H_{0}\left(\mathfrak{D}^{q}\right)$ is $q+1$.

Proof. We first suppose that $I_{g}(\xi)=R$. In this case $g$ is necessarily even. If $q \geq 0$, then Theorem 6.2 shows that $\mathfrak{D}^{q}$ is acyclic and Proposition 4.13 shows that $H_{0}\left(\mathfrak{D}^{q}\right)=0$. It follows from (4.1) and Remark 4.14 (a) that $\mathfrak{D}^{q}$ is split exact for all $q$. Henceforth, we assume that $I_{g}(\xi)$ is a proper ideal of $R$. Assertion (a) is obvious. 
(b) If $\mathfrak{D}^{q}$ has length zero, then either $g$ is odd and $-2 \leq q \leq 0$, or else, $g=1$ and $q$ is arbitrary. In either case, it is easy to see that $\mathfrak{D}_{0}^{q}=R$. See, for example, Figure 4.7 and Example 4.8.

$\left(\mathrm{b}^{\prime}\right) \quad$ If $J$ has grade one, then $g$ is necessarily even. Theorem 6.2 shows that $\mathfrak{D}^{0}$ and $\mathfrak{D}^{1}$ are acyclic. Assertion (ii) follows from (1.21) because $J$ annihilates $H_{0}\left(\mathfrak{D}^{1}\right)$. Corollary 1.26 yields that $H_{0}\left(\mathfrak{D}^{1}\right)$ is torsion-free as an $(R / J)$-module.

(c) The first assertion is Theorem 6.2. (The complex $\mathfrak{D}^{-1}$ poses no difficulty.) The second assertion follows from (4.1); and the third assertion is true, but meaningless.

(d) The assertion is true but uninteresting if $q=-1$; henceforth, we assume $q \geq 0$. We apply Proposition 1.25. Let $F_{i}$ be the radical of the $R$-ideal generated by

$$
\left\{x \in R \mid \operatorname{pd}_{R_{x}} H_{0}\left(\mathfrak{D}^{q}\right)_{x}<i\right\} .
$$

We first show that

$$
\operatorname{Pf}_{t}(\xi) \subseteq F_{g-t}
$$

for every even integer with $2 \leq t \leq g-1$. Suppose that $P$ is a prime ideal of $R$ with $\operatorname{Pf}_{t}(\xi)$ not contained in $P$. There is a basis for $G$ such that

$$
\xi_{P}=\left[\begin{array}{c|ccc}
\xi^{\prime} & & 0 & \\
\hline 0 & 0 & I_{s} \\
& -I_{s} & 0
\end{array}\right]
$$

where $t=2 s, \xi:\left(G^{\prime}\right)^{*} \rightarrow G^{\prime}$ is an alternating map and $G^{\prime}$ is a free $R_{P}$-module of rank $g-t$. We know from Proposition 4.13 that $H_{0}\left(\mathfrak{D}^{q}\left(\xi_{P}\right)\right)=S_{q}\left(\operatorname{coker} \xi_{P}\right)$. It is clear that $\xi_{P}$ and $\xi^{\prime}$ have the same cokernel; consequently, $H_{0}\left(\mathfrak{D}^{q}(\xi)\right)_{P}=$ $H_{0}\left(\mathfrak{D}^{q}\left(\xi^{\prime}\right)\right)$. Observe that $\xi^{\prime}$ satisfies $\mathrm{SPC}_{r}$ and that the length of $\mathfrak{D}^{q}\left(\xi^{\prime}\right)$ is at most $\ell$. It follows from (a) that $\mathfrak{D}^{q}\left(\xi^{\prime}\right)$ is acyclic; hence, pd $H_{0}\left(\mathfrak{D}^{q}(\xi)\right)_{P} \leq g-t-1$ and (6.18) is established.

Suppose that $g$ is odd. (In this case $J$ is the zero ideal.) We may as well assume that $q \geq 1$. The hypotheses $g \geq 3$ and $\ell\left(\mathfrak{D}^{q}\right) \leq g-r$ ensure that $r \leq g-2$. Since $\xi$ satisfies $\mathrm{SPC}_{r}$ and $I=\mathrm{Pf}_{g-1}(\xi)$, we see that grade $I=3$. Parts (d.ii) and (b) of Proposition 4.13 yield that $H_{0}\left(\mathfrak{D}^{q}\right) \cong S_{q}(I)$. As soon as we show that $H_{0}\left(\mathfrak{D}^{q}\right)$ is a torsion-free $R$-module, then we can apply Observation 1.27 in order to conclude that the natural map $S_{q}(I) \rightarrow I^{q}$ is an isomorphism. Let $i$ be an odd integer with $1 \leq i \leq \ell-1$. We see from (6.18) that

$$
\operatorname{Pf}_{g-i}(\xi) \subseteq F_{i} \subseteq F_{i+1} .
$$

Observe that $g-i$ is even and $g-i \leq g-1$. Furthermore, since $i \leq \ell-1$ and $\ell \leq g-r$, we see that $r+1 \leq g-i$. The map $\xi$ satisfies $\mathrm{SPC}_{r}$; and therefore,

$$
\text { grade } F_{i+1} \geq \operatorname{grade} F_{i} \geq i+2 .
$$

The length $\ell$ of $\mathfrak{D}^{q}$ is even, thus, grade $F_{i} \geq i+1$ for all $i$ with $1 \leq i \leq \ell$; and therefore, $H_{0}\left(\mathfrak{D}^{q}\right)$ is a torsion-free $R$-module by Proposition 1.25. 
Finally, we suppose that $g$ is even. In this case, the proper ideal $J=\operatorname{Pf}_{g}(\xi)$ is perfect of grade one. Let $i$ be an even integer with $2 \leq i \leq \ell-1$. The inclusions of (6.19) still hold. Furthermore, $g-i$ is an even integer with $r+1 \leq g-i \leq g-2$. Once again, $\xi$ satisfies $\mathrm{SPC}_{r}$ and the inequalities of (6.20) hold; and therefore $H_{0}\left(\mathfrak{D}^{q}\right)$ is a torsion-free $(R / J)$-module. The rank of $H_{0}\left(\mathfrak{D}^{q}\right)=S_{q}\left(H_{0}\left(\mathfrak{D}^{1}\right)\right)$ may be computed using Proposition 5.7.

We conclude this section by relating the complexes $\left\{\mathfrak{D}^{q}\right\}$, with $f=0$ and $g$ odd, to the resolutions of powers of a grade three Gorenstein ideal. Recall that an ideal $I$ in a commutative noetherian ring $R$ is a Gorenstein ideal of grade $c$ if $I$ is a perfect ideal of grade $c$ and $\operatorname{Ext}_{R}^{c}(R / I, R) \cong R / I$. Everyone's favorite examples of Gorenstein ideals are generated by the maximal order pfaffians of an alternating matrix. We adopt the following notation for the rest of this section.

Let $R$ be a commutative noetherian ring, $g \geq 3$ an odd integer, $G$ a free $R$-module of rank $g, \xi: G^{*} \rightarrow G$ an alternating map, and $I$ the $R$-ideal $\operatorname{Pf}_{g-1}(\xi)$. Assume that $I$ is a proper ideal of $R$ and grade $I=3$.

Theorem 6.22. ([14, Theorem 2.1]) Adopt the notation of (6.21).

(a) The ideal I is a grade three Gorenstein ideal.

(b) If $R$ is a local ring, then every grade three Gorenstein ideal in $R$ is described in (a) for some $\xi$.

The hypotheses of (6.21) ensure that $\xi$ satisfies $\mathrm{SPC}_{g-2}$; so Theorem 6.17 (c.i) and (d.ii) show that $\mathfrak{D}^{1}=\mathfrak{D}^{1}(\xi)$ is a resolution of $I$. (Of course, $\mathfrak{D}^{1}$ is exactly the same as Buchsbaum and Eisenbud's resolution of $I$; see (4.16).) Theorem 6.17 also shows that if $\xi$ is sufficiently general, then $\mathfrak{D}^{q}$ is a resolution of $I^{q}$ for all $q \geq 1$. The resolution of $I^{q}$ for $q \geq 2$ was not previously known. The next result is an example of how one can translate the statement " $\xi$ is sufficiently general" into conditions on $I$. If $M$ is a finitely generated module over a local ring, then $\mu(M)$ is the minimal number of generators of $M$.

Observation 6.23. In the notation of (6.21), then the following conditions are equivalent:

(a) $\xi$ satisfies $\mathrm{SPC}_{1}$,

(b) $\mu\left(I_{P}\right) \leq \operatorname{depth} R_{P}$ for all $P \in V(I)$.

Proof.

(a) $\Rightarrow(\mathrm{b})$. Fix $P \in V(I)$. Since $\mu\left(I_{P}\right)$ is odd, there is an integer $s$, with $3 \leq$ $g-2 s \leq g$, so that $\mu\left(I_{P}\right)=g-2 s$. We may choose a basis for $G$ so that

$$
\xi=\left[\begin{array}{c|cc}
X^{\prime} & \multicolumn{2}{|c}{0} \\
\hline 0 & 0 & I_{s} \\
& -I_{s} & 0
\end{array}\right]
$$

where every entry of the $(g-2 s) \times(g-2 s)$ alternating matrix $X^{\prime}$ is in $P R_{P}$. We see that $\mathrm{Pf}_{2 s+2}(\xi) \subseteq P$. The map $\xi$ satisfies $\mathrm{SPC}_{1}$; therefore, we conclude

$$
\mu\left(I_{P}\right)=g-2 s \leq \operatorname{grade} \operatorname{Pf}_{2 s+2}(\xi) \leq \operatorname{grade} P \leq \operatorname{depth} R_{P}
$$


(b) $\Rightarrow$ (a). Let $t$ be an even integer with $2 \leq t \leq g-1$ and let $P$ be a prime ideal with $\operatorname{Pf}_{t}(\xi) \subseteq P$ and grade $\operatorname{Pf}_{t}(\xi)=\operatorname{depth} R_{P}$. There exists an even integer $s$ with $0 \leq 2 s \leq t-2$ so that $\mathrm{Pf}_{2 s}(X)$ is not contained in $P$, but $\mathrm{Pf}_{2 s+2}(X) \subseteq P$. One can choose a basis for $G$ so that $\xi_{P}$ has the form of (6.24) where, again, every entry of $X^{\prime}$ is in $P R_{P}$. We see that $\mu\left(I_{P}\right)=g-2 s$ and we know by hypothesis that $\mu\left(I_{P}\right) \leq \operatorname{depth} R_{P}$; consequently,

$$
g-t+2 \leq g-2 s=\mu\left(I_{P}\right) \leq \operatorname{depth} R_{P}=\operatorname{gradePf}_{t}(\xi) .
$$

Adopt the notation of (6.21). Assume that $R$ is local and $I$ satisfies condition (b) of Observation 6.23. Some information about $\operatorname{depth}\left(R / I^{q}\right)$ is already known. If $q=1$, then the fact that $I$ is perfect of grade three ensures that

$$
\operatorname{depth}\left(R / I^{1}\right)=\operatorname{depth} R-3 .
$$

If $q=2$ and $R$ is regular, then Buchweitz [16, (6.2.11)] (if $R$ contains a field) and Buchweitz and Ulrich [17] (in the general case) proved that $I / I^{2}$ is a (maximal) Cohen-Macaulay $(R / I)$-module. It follows that

$$
\operatorname{depth}\left(R / I^{2}\right)=\operatorname{depth} R-3 .
$$

Huneke and Ulrich [33, Theorem 2.8] have proved that if $q \geq 3, \mu(I) \geq 4$, and $R$ is regular, then

$$
\operatorname{depth}\left(R / I^{q}\right)<\operatorname{depth} R-3 .
$$

Furthermore, they also proved [33, Lemma 2.7] that

$$
\operatorname{depth}\left(R / I^{q}\right)=\operatorname{depth} R-\mu(I)
$$

for all $q \geq \mu(I)-2$, provided $R$ is Gorenstein. We are able to capture all of these results (without any extraneous hypotheses on the ring $R$ ) and find $\operatorname{depth}\left(R / I^{q}\right)$ for all intermediate values of $q$. Notice that the pattern

$$
\operatorname{depth}\left(R / I^{q}\right)=\operatorname{depth}\left(R / I^{q+1}\right),
$$

which is initiated at $q=1$, is repeated forever for all odd $q$. (For further results about $\left\{\operatorname{depth}\left(R / K^{q}\right)\right\}$ for more general ideals $K$, consult $[18,5,27]$.)

Theorem 6.25. Adopt the notation of (6.21). If $\mu\left(I_{P}\right) \leq \operatorname{depth} R_{P}$ for all $P \in$ $V(I)$, then the following statements hold.

(a) For all $q \geq 0, \mathfrak{D}^{q}$ is a resolution of $I^{q} \cong S_{q}(I)$.

(b) If $R$ is a local ring and $\mu(I)=g$, then $\mathfrak{D}^{q}(\xi)$ is a minimal resolution of $I^{q} \cong S_{q}(I)$, and $\operatorname{depth}\left(R / I^{q}\right)=\operatorname{depth} R-s$ where

$$
s=\min \{g, 1+2 \llbracket(q+1) / 2 \rrbracket\} ;
$$

in other words, $s$ is the largest odd integer with

$$
s \leq \min \{\mu(I), q+2\} .
$$


Proof.

(a) Observation 6.23 shows that Theorem 6.17 applies.

(b) The hypothesis $\mu(I)=g$ ensures that the ideal $I_{1}(\xi)$ is contained in the maximal ideal of $R$; and therefore, $\mathfrak{D}^{q}$ is a minimal resolution of $J$. (See Observation 4.28.) The projective dimension of $I^{q}$, which is the same as $\ell\left(\mathfrak{D}^{q}\right)$, is given in Observation $4.3(\mathrm{~g})$. The proof is completed by applying the Auslander-Buchsbaum equation since

$$
\operatorname{pd}_{R}\left(R / I^{q}\right)=1+\operatorname{pd}_{R} I^{q} .
$$




\section{SeCtion 7. ACYCLICITy IN THE GENERIC CASE.}

In this section

$R_{0}$ is a commutative noetherian ring; $\rho=\left[\begin{array}{ll}X & Y\end{array}\right]$ is a generic almost alternating map of shape $(g, f)$ over the polynomial ring $R=R_{0}[X, Y]$; $e=f+g$; the complexes $\mathfrak{D}^{q}=\mathfrak{D}^{q}(\rho)$ are defined in section 2 ; the ideals $J=J(\rho)$ and $I=I(\rho)$ are defined in $(4.10) ; \bar{R}=R / J$; and $M=\operatorname{coker} \rho$.

In Proposition 4.13 we proved that there is an $\bar{R}$-module surjection

$$
H_{0}\left(\mathfrak{D}^{q}\right) \rightarrow I^{q} \bar{R}
$$

for all $q \geq 0$. In this section we prove

Theorem 7.3. Adopt the notation of (7.1).

(a) The ideal $J$ is perfect. The grade of $J$ is $f+1$ if $f=0$ and $g$ is even. In all other cases, the grade of $J$ is $f$.

(b) If $\ell\left(\mathfrak{D}^{q}\right)=f$, then

(i) the complex $\mathfrak{D}^{q}$ is acyclic,

(ii) the $R$-module $H_{0}\left(\mathfrak{D}^{q}\right)$ is perfect of projective dimension $f$, and

(iii) the $(R / J)$-module $H_{0}\left(\mathfrak{D}^{q}\right)$ is torsion-free and has rank one.

(c) If $q \geq-1$, then

(i) the complex $\mathfrak{D}^{q}$ is acyclic,

(ii) $H_{j}\left(\mathfrak{D}^{f-2-q}\right)=\operatorname{Ext}_{R}^{f-j}\left(H_{0}\left(\mathfrak{D}^{q}\right), R\right)$ for all $j$, and

(iii) $H_{j}\left(\mathfrak{D}^{f-2-q}\right)=0$ for $j \geq 1$.

(d) If $q \geq-1$, then $H_{0}\left(\mathfrak{D}^{q}\right)$ is a torsion-free $(R / J)$-module with

$$
\operatorname{rank} H_{0}\left(\mathfrak{D}^{q}\right)= \begin{cases}q+1 & \text { if } g \text { is even and } f=0 \\ 1 & \text { in all other cases. }\end{cases}
$$

(e) If $q \geq 0$, and either, $g$ is odd, or else, $g$ is even and $f$ is positive, then the surjection of (7.2) is an isomorphism.

Proof. We induct on $f$. The case $f=0$ has been established in Theorem 6.17 (using Observation 5.13). Henceforth, we assume that $f \geq 1$ is a fixed integer. Our proof has two parts. First we assume that

$\mathfrak{D}^{q}(\rho)$ is acyclic for all $q \geq 0$ and all almost generic alternating maps $\rho$ of shape $(g, f)$ where $g \geq 1$ is arbitrary.

Let $\rho$ be a fixed generic almost alternating map of shape $(g, f)$. Using (7.4) we prove that $(\mathrm{a})-(\mathrm{e})$ also hold for $\rho$.

(a) The hypothesis (7.4) shows that $\mathfrak{D}^{0}$ is a length $f$ resolution of $H_{0}\left(\mathfrak{D}^{0}\right)=R / J$ by free $R$-modules. Observation 5.13, together with Corollary 5.5, shows that grade $J \geq f$. We conclude from (1.21) that $J$ is perfect of grade $f$. 
(b) Suppose first that $q \geq 0$ and $\ell\left(\mathfrak{D}^{q}\right)=f$. Assertion (i) is contained in hypothesis (7.4). Thus, $\mathfrak{D}^{q}$ is a length $f$ resolution of the $R$-module $H_{0}\left(\mathfrak{D}^{q}\right)$, and $\operatorname{pd}_{R} H_{0}\left(\mathfrak{D}^{q}\right) \leq f$. On the other hand, we know, from Proposition 4.13, that the grade $f$ ideal $J$ annihilates $H_{0}\left(\mathfrak{D}^{q}\right)$. Assertion (ii) is now established, and (iii) follows by way of Corollary 1.26 and Proposition 5.7.

Now suppose that $q \leq-1$ and $\ell\left(\mathfrak{D}^{q}\right)=f$. We see, from (4.1), that

$$
\mathfrak{D}_{j}^{q} \cong\left(\mathfrak{D}_{f-j}^{f-2-q}\right)^{*}
$$

It follows that $\mathfrak{D}^{f-2-q}$ also has length $f$. The preceding paragraph may be applied to $\mathfrak{D}^{f-2-q}$ because $0 \leq f-2-q$. It follows that the dual of $\mathfrak{D}^{f-2-q}$, which up to shift is $\mathfrak{D}^{q}$, also resolves a perfect $R$-module. Notice that (7.5) shows that the non-zero modules in $\mathfrak{D}^{q}$ are $\mathfrak{D}_{j}^{q}$ for $0 \leq j \leq f$. We compute the rank of $H_{0}\left(\mathfrak{D}^{q}\right)$. If $P$ is a prime of $R$ with $P \in \operatorname{Ass}(R / J)$, then the grade of $P$ is $f$. Proposition 5.7 shows that

$$
H_{0}\left(\mathfrak{D}^{f-2-q}\right)_{P}=(R / J)_{P}
$$

and that $J_{P}$ is generated by a regular sequence of length $f$. It follows that

$$
H_{0}\left(\mathfrak{D}^{q}\right)_{P}=\operatorname{Ext}_{R}^{f}\left(H_{0}\left(\mathfrak{D}^{f-2-q}\right), R\right)_{P}=(R / J)_{P}
$$

and hence, $H_{0}\left(\mathfrak{D}^{q}\right)$ is an $(R / J)$-module of rank one.

(c) The complex $\mathfrak{D}^{-1}$ has length $f$; so (i) follows from (7.4) and (b). Assertion (ii) can be read from (7.5); and (iii) follows from (ii) because the grade $f$ ideal $J$ is contained in the annihilator of $H_{0}\left(\mathfrak{D}^{q}\right)$.

(d) If $-1 \leq q \leq f-1$, then Observation 4.3 (e) shows that $\ell\left(\mathfrak{D}^{q}\right)=f$; and therefore, (b) shows that $H_{0}\left(\mathfrak{D}^{q}\right)$ is torsion-free. Henceforth, we assume that $q \geq f$. For each integer $i$, let $F_{i}$ be the radical of the $R$-ideal generated by

$$
\left\{x \in R \mid \operatorname{pd}_{R_{x}} H_{0}\left(\mathfrak{D}^{q}\right)_{x}<i\right\} .
$$

The length of $\mathfrak{D}^{q}$ is bounded by $e-1$; thus, according to Proposition 1.25, we may show that $H_{0}\left(\mathfrak{D}^{q}\right)$ is torsion-free by proving that

$$
\text { grade } F_{i} \geq i+1 \quad \text { for all } i \text { with } \quad f+1 \leq i \leq e-1 \text {. }
$$

Let $i$ be a fixed integer in the above range, and let $P$ be a prime ideal which contains $F_{i}$. It suffices to show that

$$
I_{e-1}(\rho) \subseteq P
$$

(Indeed, if (7.7) holds, then the grade of $P$ is at least $i+1$ because $1 \leq e-i \leq$ $g-1$ and $\rho$ satisfies $\mathrm{SMC}_{1}$ by Observation 5.13.) If (7.7) does not hold, then $I_{e-1}\left(\rho \otimes R_{P}\right)=R_{P}$. On the other hand, $J \subseteq F_{i}$, and therefore, $I_{g}(\rho) \subseteq P$ by Corollary 5.5. Lemma 5.6 and the note following it show that there is an almost alternating map $\rho^{\prime}$, over $R_{P}$, of shape $\left(g^{\prime}, f\right)$, with $g^{\prime}<g$ and $g^{\prime}+f \leq i$, such that $H_{0}\left(\mathfrak{D}^{q}(\rho)\right)=H_{0}\left(\mathfrak{D}^{q}\left(\rho^{\prime}\right)\right)$. Furthermore, since $\rho^{\prime}$ is a localization of a generic 
almost alternating map of shape $\left(g^{\prime}, f\right)$, we know, from $(7.4)$, that $\mathfrak{D}^{q}\left(\rho^{\prime}\right)$ is acyclic. It follows that

$$
\operatorname{pd}_{R_{P}} H_{0}\left(\mathfrak{D}^{q}\right)_{P}=\operatorname{pd}_{R_{P}} H_{0}\left(\mathfrak{D}^{q}\left(\rho^{\prime}\right)\right) \leq g^{\prime}+f-1<i .
$$

This last inequality contradicts the assumption that $F_{i} \subseteq P$; therefore, $H_{0}\left(\mathfrak{D}^{q}\right)$ is a torsion-free $(R / J)$-module. The rank of $H_{0}\left(\mathfrak{D}^{q}\right)$ may be read from Proposition 5.7.

(e) We apply Observation 1.27. Use (a) and Proposition 5.14 in order to see that

$$
\text { grade } I^{q} \bar{R} \geq \operatorname{grade}(I+J)-\text { grade } J \geq(f+1)-f=1 .
$$

(The first inequality is due to the fact that $J$ is perfect; see [9, Proposition 16.18].) The $\bar{R}$-module $H_{0}\left(\mathfrak{D}^{q}\right)$ is torsion-free of rank one by (d).

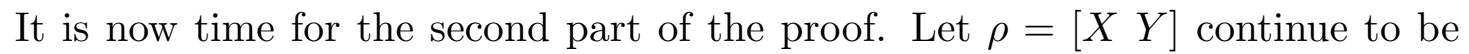
a fixed generic almost alternating map of shape $(g, f)$ and let $q \geq 0$ be a fixed integer. We now suppose that all five assertions $(a)-(e)$ hold for all generic almost alternating maps $\rho^{\prime}$ of shape $(g, f-1)$; and we prove that $\mathfrak{D}^{q}$ is acyclic. Let $Y^{\prime}$ be the matrix which is obtained by removing the last column from the matrix $Y, \rho^{\prime}$ the generic almost alternating map $\left[X Y^{\prime}\right], \mathfrak{D}^{\prime q}=\mathfrak{D}^{q}\left(\rho^{\prime}\right), J^{\prime}=J\left(\rho^{\prime}\right)$, and $M^{\prime}=$ coker $\rho^{\prime}$. Since $q \geq-1$, we know, from the induction hypothesis, that $\mathfrak{D}^{\prime q}$ and $\mathfrak{D}^{\prime q-1}$ are both acyclic. The long exact sequence of homology in (4.25) yields that $H_{i}\left(\mathfrak{D}^{q}\right)=0$ for all $i \geq 2$. Proposition 4.23 also shows that

$$
\begin{aligned}
0 & \rightarrow H_{1}\left(\mathfrak{D}^{q}\right) \rightarrow S_{q-1}\left(M^{\prime}\right) \stackrel{\rho(\phi)}{\longrightarrow} S_{q}\left(M^{\prime}\right) \quad \text { for } q \geq 1, \text { and } \\
0 & \rightarrow H_{1}\left(\mathfrak{D}^{0}\right) \rightarrow H_{0}\left(\mathfrak{D}^{\prime-1}\right) \longrightarrow J / J^{\prime} \rightarrow 0
\end{aligned}
$$

are exact sequences (for $\phi=\phi_{f}$ ).

There are two cases for us to consider. We first suppose that $f-1=0$ and $g$ is even. In this case $\mathfrak{D}^{\prime-1}$ is the zero complex; thus, $H_{0}\left(\mathfrak{D}^{\prime-1}\right)=H_{1}\left(\mathfrak{D}^{0}\right)=0$. Furthermore, the symmetric algebra $S=S_{\left(R / J^{\prime} R\right)}\left(M^{\prime}\right)$ is a familiar object. Recall that $R / J^{\prime}=R_{0}[X, Y] / \operatorname{Pf}_{g}(X)$ where $X_{g \times g}$ is a generic alternating matrix and $Y_{g \times 1}$ is a generic matrix. Since

$$
\left(R / J^{\prime} R\right)^{g} \stackrel{X}{\longrightarrow}\left(R / J^{\prime} R\right)^{g} \longrightarrow M^{\prime}
$$

is a presentation of the $\left(R / J^{\prime} R\right)$-module $M^{\prime}$; it follows that

$$
S=\frac{R_{0}[X, Y, T]}{I_{1}(T X)+\operatorname{Pf}_{g}(X)}
$$

where $T_{1 \times g}$ is a generic matrix. It is clear that $\rho(\phi)$ is the element $I_{1}(T Y)$ in $S$. We see from Lemma 7.10 that $\rho(\phi)$ is a non-zero-divisor on $S$; and therefore, $H_{1}\left(\mathfrak{D}^{q}\right)=0$ for all $q \geq 1$. The proof is complete if $f=1$ and $g$ is even.

Finally, we consider the case where, either, $g$ is odd and $f-1 \geq 0$, or else, $g$ is even and $f-1 \geq 1$. Fix $q \geq 1$. In this case, the induction hypothesis guarantees that for $i$ equal to either $q-1$ or $q$ the map

$$
H_{0}\left(\mathfrak{D}^{\prime i}\right)=S_{i}\left(M^{\prime}\right) \rightarrow\left(I^{\prime}\right)^{i}
$$


of (7.2) is an isomorphism where $I^{\prime}=I\left(R / J^{\prime}\right)$. In the exact sequence of $(7.8)$ we are required to interpret $\rho(\phi)$ as an element of $S_{1}\left(M^{\prime}\right) \subseteq S$. We see from (4.15) that the isomorphism

$$
S_{1}\left(M^{\prime}\right) \longrightarrow I^{\prime}
$$

carries $\rho(\phi)$ to the element

$$
z=\sum_{i=1}^{g} y_{i f} \widetilde{X}_{\langle i\rangle}+J^{\prime} \quad \text { of } R / J^{\prime}
$$

We conclude from $(1.18)$ or $(1.20)$ that $z= \pm \operatorname{pf}(\widetilde{\widetilde{X}})+J^{\prime}$ where $\widetilde{\widetilde{X}}$ is defined in (5.17). The ideal $J^{\prime}$ is perfect of grade $f-1$ by the induction hypothesis. Thus, if $P$ is a prime ideal of $R$ with $P \in \operatorname{Ass}\left(R / J^{\prime}\right)$, then grade $P=f-1$. Proposition 5.16 shows that $z \notin P / J^{\prime}$; and therefore, $z$ is a regular element of $R / J^{\prime}$. In the present notation the exact sequence (7.8) is

$$
0 \rightarrow H_{1}\left(\mathfrak{D}^{q}\right) \rightarrow\left(I^{\prime}\right)^{q-1} \stackrel{z}{\rightarrow}\left(I^{\prime}\right)^{q} .
$$

It follows that $H_{1}\left(\mathfrak{D}^{q}\right)=0$ for $q \geq 1$. Finally, we show that $H_{1}\left(\mathfrak{D}^{0}\right)=0$ by applying Observation 1.27 in order to see that

$$
H_{0}\left(\mathfrak{D}^{\prime-1}\right) \rightarrow\left(J / J^{\prime}\right)
$$

is an isomorphism. We know that $\left(J / J^{\prime}\right)$ has positive grade because $z \in\left(J / J^{\prime}\right)$. The $\left(R / J^{\prime}\right)$-module $H_{0}\left(\mathfrak{D}^{\prime-1}\right)$ is torsion-free of rank one by the induction hypothesis.

Lemma 7.10. Let $R$ be a commutative noetherian ring, $g$ an even integer, $T_{1 \times g}$ and $Y_{g \times 1}$ generic matrices, and $X_{g \times g}$ a generic alternating matrix. If $A$ is the ideal $I_{1}(T X)+\operatorname{Pf}_{g}(X)$ in the ring $\widetilde{R}=R[T, X, Y]$, then the element $T Y$ is regular on $\widetilde{R} / A$.

Proof. Let $B$ be the ideal $I_{1}(T X)+\operatorname{Pf}_{g}(X)$ in the $\operatorname{ring} R[T, X]$. Then $B$ is a deviation two Gorenstein ideal. These ideals were introduced by Huneke and Ulrich [30, Lemma 5.12] and they were resolved in [40]. (Other resolutions may be found in [36] and [51].) In particular, $B$ is a perfect ideal of grade $g-1$. Therefore $I_{1}(T)$ is not contained in any associated prime of $R[T, X] / B$. In other words, $T_{1}, \ldots, T_{g}$ generate an ideal of positive grade in $R[T, X] / B$; and hence, by Hochster's general grade reduction [24], the element $\sum T_{i} Y_{i}=T Y$ is regular on $(R[T, X] / B)[Y]=$ $\widetilde{R} / A$.

Remark 7.11. Our proof in Theorem 7.3 (b.iii) and (d) that $H_{0}\left(\mathfrak{D}^{q}\right)$ is a torsionfree $(R / J)$-module used Proposition 1.25; and therefore, also proved the slightly stronger conclusion that $H_{0}\left(\mathfrak{D}^{q}\right)_{P}$ is a torsion-free $(R / J)_{P}$-module for all prime ideals $P$ of $R / J$. We return to this theme in Proposition 9.2. 


\section{Section 8. ACyClicity in the NON-Generic CASE.}

The following hypotheses are used throughout the entire section:

$\rho=[\xi \psi]: G^{*} \oplus F \rightarrow G$ is an almost alternating map of shape $(g, f)$ over a commutative noetherian ring $R$; $e=f+g$; the complexes $\mathfrak{D}^{q}=\mathfrak{D}^{q}(\rho)$ are defined in section 2; and the ideal $J=J(\rho)$ is defined in (4.10).

Let $\left[\begin{array}{ll}X & Y\end{array}\right]$ be a matrix representation of $\rho$, and let $\widetilde{\rho}=\left[\begin{array}{ll}\widetilde{X} & \widetilde{Y}\end{array}\right]$ be a generic almost alternating matrix with the same shape as $\rho$. The entries $\widetilde{x}_{i j}$ and $\widetilde{y}_{i j}$ of $\widetilde{X}$ and $\widetilde{Y}$ are indeterminates over $R$; whereas the entries $x_{i j}$ and $y_{i j}$ of $X$ and $Y$ are elements of $R$. The ideal $\widetilde{J}=J(\widetilde{\rho})$ of the polynomial ring $R[\widetilde{X}, \widetilde{Y}]$ is perfect. Its grade is given in Theorem 7.3 (a). The ideal $J$ is the image of $\widetilde{J}$ under the $R$-algebra homomorphism $R[\widetilde{X}, \widetilde{Y}] \rightarrow R$ which sends $\widetilde{x}_{i j}$ to $x_{i j}$ and $\widetilde{y}_{i j}$ to $y_{i j}$. We know, from Proposition 1.22, that if $J$ is a proper ideal in $R$, then grade $J \leq$ grade $\widetilde{J}$. Moreover, we know from Corollary 5.5 that the $R$-ideals $J$ and $I_{g}(\rho)$ have the same radical. It follows that if $I_{g}(\rho)$ is a proper ideal of $R$, then

$$
\text { grade } I_{g}(\rho) \leq \begin{cases}f+1 & \text { if } f=0 \text { and } g \text { is even } \\ f & \text { in all other cases. }\end{cases}
$$

We are now ready to prove the main theorem in this paper. The conditions WMC and SMC are defined in (5.8).

Theorem 8.3. Adopt the notation of (8.1). If $I_{g}(\rho)=R$, then every complex $\mathfrak{D}^{q}$ is split exact. If $I_{g}(\rho)$ is a proper ideal of $R$ and equality holds in (8.2), then the following statements hold.

(a) The ideal $J$ is perfect. The grade of $J$ is $f+1$ if $f=0$ and $g$ is even. In all other cases, the grade of $J$ is $f$.

(b) If $\ell\left(\mathfrak{D}^{q}\right)=f$, then the following statements hold.

(i) The complex $\mathfrak{D}^{q}$ is acyclic.

(ii) The $R$-module $H_{0}\left(\mathfrak{D}^{q}\right)$ is perfect of projective dimension $f$.

(iii) The $(R / J)$-module $H_{0}\left(\mathfrak{D}^{q}\right)$ is torsion-free. If grade $I_{g-1}(\rho) \geq f+1$, then $H_{0}\left(\mathfrak{D}^{q}\right)$ has rank one.

(c) If $\rho$ satisfies $\mathrm{WMC}_{r}$ for some $r$ with $1 \leq r \leq g-1$, and $q \geq-1$ is an integer with $\ell\left(\mathfrak{D}^{q}\right) \leq e-r$, then

(i) the complex $\mathfrak{D}^{q}$ is acyclic,

(ii) $H_{j}\left(\mathfrak{D}^{f-2-q}\right)=\operatorname{Ext}_{R}^{f-j}\left(H_{0}\left(\mathfrak{D}^{q}\right), R\right)$ for all $j$, and

(iii) $H_{j}\left(\mathfrak{D}^{f-2-q}\right)=0$ for $j \geq 1$.

(d) If $\rho$ satisfies $\mathrm{SMC}_{r}$ for some $r$ with $1 \leq r \leq g-1$, and $q \geq-1$ is an integer with $\ell\left(\mathfrak{D}^{q}\right) \leq e-r$, then $H_{0}\left(\mathfrak{D}^{q}\right)$ is a torsion-free $(R / J)$-module of rank one.

Note. The length, $\ell\left(\mathfrak{D}^{q}\right)$, of $\mathfrak{D}^{q}$ is given in Observation 4.3. Recall that the inequality $f \leq \ell \leq e-1$ holds in all cases, except for the one trivial complex $\mathfrak{D}^{-1}$ when $f=0$ and $g$ is even. Recall, also, that $\ell\left(\mathfrak{D}^{q}\right)=f$ in each of the following situations: $f \geq 1$ and $-1 \leq q \leq f-1$; or $g=1$ and $q$ is arbitrary; or $g$ is odd, $-2 \leq q \leq 0$, and $f=0$. If $\rho$ is a generic almost alternating map, then the minor and 
pfaffian conditions which $\rho$ satisfies are recorded in Observation 5.13; in particular, $\rho$ satisfies $\mathrm{SMC}_{1}$ if $f \geq 1$. Notice that if $f=0$, then Theorem 6.17 is a better result than Theorem 8.3 because, according to Corollary $5.11, \mathrm{MC}_{r} \Rightarrow \mathrm{PC}_{r}$ for $1 \leq r \leq g-1$.

Proof of Theorem 8.3. If $f=0$, then the present result is implied by Theorem 6.17. Henceforth, we assume that $f \geq 1$. We continue to use " $\sim$ " as described in the first paragraph of this section. We next consider the case where $I_{g}(\rho)=R$. Corollary 5.5 shows that

$$
\widetilde{J}+\operatorname{Ker}(R[\widetilde{X}, \widetilde{Y}] \rightarrow R)=R[\widetilde{X}, \widetilde{Y}]
$$

If $q \geq 0$, then Proposition 4.13 shows that $\widetilde{J}$ annihilates $H_{0}\left(\mathfrak{D}^{q}(\widetilde{\rho})\right)$; and consequently,

$$
\operatorname{Tor}_{i}^{R[\widetilde{X}, \widetilde{Y}]}\left(R, H_{0}\left(\mathfrak{D}^{q}(\widetilde{\rho})\right)\right)=0
$$

for all $i$. This and Theorem 7.3 (c.i) imply that $\mathfrak{D}^{q}$ is split exact for $q \geq 0$; and therefore, (4.1) shows that $\mathfrak{D}^{q}$ is split exact for all $q$. Henceforth, we assume that $I_{g}(\rho)$ and $J$ are proper ideals of $R$. Assertions (a) and (b) hold for $\widetilde{\rho}$ by Theorem 7.3. It follows from Proposition 1.22, Corollary 1.26, and Proposition 5.7 that they also hold for $\rho$. (The argument of (7.6) shows that the rank of $H_{0}\left(\mathfrak{D}^{q}\right)$ is one even if $q \leq-1$.) Assertions (c.ii) and (c.iii) follow from (c.i) exactly as in the proof of Theorem 7.3. The rank of $H_{0}\left(\mathfrak{D}^{q}\right)$ in (d) is given by Proposition 5.7 and (7.6). (Notice that the hypothesis that $\rho$ satisfies $\mathrm{SMC}_{r}$ for some $r \leq g-1$ implies that grade $I_{g-1}(\rho) \geq f+2$. In particular, the case $g$ is even and $f=0$ is not treated in $(\mathrm{d})$ of Theorem 8.3. The appropriate result in this case is Theorem $6.17(\mathrm{~d})$.)

Let $q$ and $r$ be fixed integers with $1 \leq r \leq g-1, q \geq f$, and $\ell\left(\mathfrak{D}^{q}\right) \leq e-r$. We must prove

(c.i) if $\rho$ satisfies $\mathrm{WMC}_{r}$, then $\mathfrak{D}^{q}$ is acyclic, and

(d) if $\rho$ satisfies $\mathrm{SMC}_{r}$, then $H_{0}\left(\mathfrak{D}^{q}\right)$ is a torsion-free $(R / J)$-module.

These statements may be proved locally; so it suffices to assume that $(R, \mathfrak{m})$ is a local ring. Let $\mathfrak{M}$ be the maximal ideal $\left(\mathfrak{m}, \widetilde{x}_{i j}-x_{i j}, \widetilde{y}_{i j}-y_{i j}\right)$ in the polynomial ring $R[\widetilde{X}, \widetilde{Y}]$, and let $\widetilde{R}$ be the local ring $R[\widetilde{X}, \widetilde{Y}]_{\mathfrak{M}}$. Form the $\widetilde{R}$-complexes $\widetilde{\mathfrak{D}}^{q}$, and the $\widetilde{R}$-ideal $\widetilde{J}$, using the almost alternating map $\widetilde{\rho}=[\tilde{X} \widetilde{Y}]$ over the ring $\widetilde{R}$. We know, from Theorem 7.3 and Remark 7.11, that

$\widetilde{J}$ is a perfect ideal of grade $f, \widetilde{\mathfrak{D}}^{q}$ is acyclic, and $H_{0}\left(\widetilde{\mathfrak{D}}^{q}\right)$ is a torsionfree $(\widetilde{R} / \widetilde{J})$-module.

Let $Z$ be the ideal $\left(\widetilde{x}_{i j}-x_{i j}, \widetilde{y}_{i j}-y_{i j}\right)$ in $\widetilde{R}$. It is clear that

$$
\widetilde{R} / Z=R, \quad \widetilde{\rho} \otimes(\widetilde{R} / Z)=\rho, \quad \text { and } \quad \widetilde{\mathfrak{D}}^{q} \otimes(\widetilde{R} / Z)=\mathfrak{D}^{q}
$$

The proof proceeds by induction on $g$. For $g=1$, the claim follows from part (b). So, assume that $g \geq 2$. The ideal $Z$ is generated by a regular $\widetilde{R}$-sequence. We know, from Observation 5.13, that $\widetilde{\rho}$ satisfies the condition $\mathrm{SMC}_{1}$; thus, we 
may apply Lemma 8.8 and choose a regular sequence $z_{1}, \ldots, z_{d}$ which generates $Z$ and which also has the property that

$$
\widetilde{\rho} \otimes \frac{\widetilde{R}}{\left(z_{1}, \ldots, z_{i}\right)}
$$

satisfies $\mathrm{SMC}_{r}$ for $0 \leq i \leq d-1$. The proof is completed by induction on $i$. Assume that the conclusion holds for the almost alternating map on line (8.6); we show that the conclusion also holds for

$$
\widetilde{\rho} \otimes \frac{\widetilde{R}}{\left(z_{1}, \ldots, z_{i}, z_{i+1}\right)} .
$$

A change of notation is in order. Instead of assuming that $\widetilde{\rho}$ is generic, we assume that $\widetilde{\rho}$ satisfies $\mathrm{SMC}_{r}, \widetilde{\rho}$ satisfies (8.4), and $Z$ is a regular element of $\widetilde{R}$ for which (8.5) holds. We also assume that $\rho$ satisfies $\mathrm{WMC}_{r}$. We must prove that $\mathfrak{D}^{q}$ is acyclic. Furthermore, if $\rho$ satisfies $\mathrm{SMC}_{r}$, then we must prove that $H_{0}\left(\mathfrak{D}^{q}\right)$ is a torsion-free $(R / J)$-module.

Parts (a) and (b) show that $J$ is a perfect ideal of grade $f$ and that $\mathfrak{D}^{0}=$ $\widetilde{\mathfrak{D}}^{0} \otimes(\widetilde{R} / Z)$ is acyclic. It follows that

$$
\operatorname{Tor}_{i}^{\widetilde{R}}((\widetilde{R} / Z),(\widetilde{R} / \widetilde{J}))=0
$$

for all positive $i$; and therefore, since $Z$ is regular on $\widetilde{R}$, we know that $Z$ is regular on $\widetilde{R} / \widetilde{J}$. The element $Z$ is also regular on the torsion-free $(\widetilde{R} / \widetilde{J})$-module $H_{0}\left(\widetilde{\mathfrak{D}}^{q}\right)$; hence, $\widetilde{\mathfrak{D}}^{q} \otimes(\widetilde{R} / Z)=\mathfrak{D}^{q}$ is acyclic.

Assume that $\rho$ satisfies $\mathrm{SMC}_{r}$. For each integer $i$, let $F_{i}$ be the radical of the $R$-ideal generated by:

$$
\left\{x \in R \mid \operatorname{pd}_{R_{x}} H_{0}\left(\mathfrak{D}^{q}\right)_{x}<i\right\} .
$$

According to Proposition 1.25, we can prove that $H_{0}\left(\mathfrak{D}^{q}\right)$ is a torsion-free $(R / J)$-module by proving that

$$
\text { grade } F_{i} \geq i+1 \quad \text { for all } i \text { with } \quad f+1 \leq i \leq \ell=\ell\left(\mathfrak{D}^{q}\right) .
$$

Let $i$ be a fixed integer in the above range, and let $P$ be a prime ideal which contains $F_{i}$. It suffices to show that

$$
I_{e-i}(\rho) \subseteq P .
$$

Indeed, if (8.7) holds, then the grade of $P$ is at least $i+1$ because $\ell \leq e-r$ and $\rho$ satisfies $\mathrm{SMC}_{r}$. If (8.7) does not hold, then we reach the contradiction

$$
\operatorname{pd}_{R_{P}} H_{0}\left(\mathfrak{D}^{q}\right)_{P}=\operatorname{pd}_{R_{P}} H_{0}\left(\mathfrak{D}^{q}\left(\rho^{\prime}\right)\right) \leq g^{\prime}+f-1<i
$$

exactly as in the proof of Theorem 7.3 (d). The only change is that, in Theorem 7.3, $\rho^{\prime}$ is a localization of a generic almost alternating map; whereas, in the present proof, $\rho^{\prime}$ satisfies $\mathrm{SMC}_{r}$. Since $g^{\prime}<g$, we obtain the above inequality for $\operatorname{pd}_{R_{P}} H_{0}\left(\mathfrak{D}^{q}\left(\rho^{\prime}\right)\right)$ from our induction hypothesis applied to $\rho^{\prime}$. 
Lemma 8.8. Let $(R, \mathfrak{m})$ be a noetherian local ring and let $Z$ be an ideal of $R$ which is generated by a regular sequence of length $d$. Suppose that $r_{1}, \ldots, r_{s}$ are non-negative integers and $W_{1}, \ldots, W_{s}$ are ideals of $R$ with grade $W_{t} \geq r_{t}$ and grade $W_{t}(R / Z) \geq r_{t}-1$, for all $t$ with $1 \leq t \leq s$. Then there is an $R$-sequence $z_{1}, \ldots, z_{d}$ which generates $Z$ with grade $W_{t}\left(R /\left(z_{1}, \ldots, z_{i}\right)\right) \geq r_{t}$ for all $t$ and for all $i$ with $1 \leq i \leq d-1$.

Proof. There is nothing to prove if $d=1$. It suffices to assume that $d \geq 2$ and to find a minimal generator $z$ of $Z$ with grade $W_{t}(R /(z)) \geq r_{t}$ for all $t$.

For each $t$, let $\mathbf{x}^{(t)}$ be an $R$-sequence of length $r_{t}$ in $W_{t}$. Let $S$ be the set of all prime ideals in $R$ such that there is an integer $t, 1 \leq t \leq s$, with $W_{t} \subseteq P$ and $P$ an associated prime of $R /\left(\mathbf{x}^{(t)}\right)$. Observe that $Z$ is not contained in any of the prime ideals of $S$. Indeed, if $Z \subseteq P$ for some $P \in S$, then

$$
Z+W_{t} \subseteq P \in \operatorname{Ass}\left(\frac{R}{\left(\mathbf{x}^{(t)}\right)}\right)
$$

for some $t$. It follows that grade $\left(Z+W_{t}\right)=r_{t}$. On the other hand, $Z$ is generated by a regular sequence of length $d \geq 2$; thus,

$$
\operatorname{grade} \frac{\left(Z+W_{t}\right)}{Z}=\operatorname{grade}\left(Z+W_{t}\right)-d=r_{t}-d \leq r_{t}-2
$$

This inequality contradicts the hypothesis that the ideal $W_{t}(R / Z)$ had grade at least $r_{t}-1$. We apply the prime avoidance lemma to find an element $z$ of $Z$ with $z \notin \mathfrak{m} Z$ and $z \notin P$ for any $P$ in $S$. Fix $t$. It follows, from the choice of $z$, that the grade of $(z)+W_{t}$ is at least $r_{t}+1$. On the other hand, since $z$ is regular on $R$, we know that the grade of $W_{t}(R /(z))$ is one less than the grade of $(z)+W_{t}$.

Now that we know that $J$ is perfect, it is easy to make the calculation which shows that $J$ is a residual intersection. We return to this theme in Section 10.

Corollary 8.9. Adopt the notation of (8.1) with $J$ a proper ideal and either $g$ odd or $f$ positive. If $g$ is even, then fix a basis element $\phi_{1}$ of $F$. Form the ideals $A=A(\rho)$ and $I=I(\rho)$ of Definition 4.10. If grade $J \geq f$, grade $I \geq 3$, and grade $(I+J) \geq f+1$, then

(a) $(I \cap J)=A$, and

(b) $(A: I)=J$.

Note. If $\rho$ is a generic almost alternating map, then, according to Observation 5.13, (1.24) and Proposition 5.14, the grade hypotheses are all satisfied.

Proof. If $g=1$, then $I=R$ and $A=J$. The result is true but not interesting. Henceforth, we assume $g \geq 2$.

We first observe that $H_{0}\left(\mathfrak{D}^{1}\right)$ is a torsion-free $(R / J)$-module of rank one. Indeed, we know from Observation 4.3 that

$$
\ell\left(\mathfrak{D}^{1}\right)= \begin{cases}f & \text { if } f \geq 2 \\ 2=e-(g-1) & \text { if } f=1 \\ 2=e-(g-2) & \text { if } f=0\end{cases}
$$


Recall, from Observation 5.12, that

$$
\operatorname{grade} I_{g-1}(\rho) \geq \operatorname{grade}(I+J) \geq \max \{3, f+1\} .
$$

If $f \geq 2$, then Theorem 8.3 (b) completes this part of the proof. If $f=1$, then (8.10) shows that $\rho$ satisfies $\mathrm{SMC}_{g-1}$. If $f=0$, then Observation 5.10 shows that

$$
\text { grade } I_{g-2}(\rho)=\text { grade } I_{g-1}(\rho)=\operatorname{grade} I=3
$$

hence, $\rho$ satisfies $\mathrm{SMC}_{g-2}$. If $f \leq 1$, then Theorem 8.3 (d) completes this part of the proof.

The proof of Proposition 4.13 (d.iv) shows that there is a commutative triangle

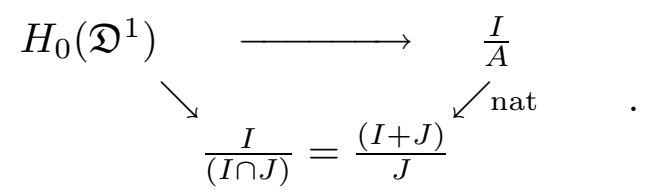

The horizontal map is an isomorphism by Proposition 4.13 (d.ii). The map labeled "nat" is the natural map induced by the inclusion $A \subseteq(I \cap J)$. Observation 1.27 shows that the other map is also an isomorphism because

$$
\text { grade } \frac{I+J}{J} \geq \operatorname{grade}(I+J)-\text { grade } J \geq(f+1)-f=1,
$$

since $J$ is perfect of grade $f$. It follows that "nat" is also an isomorphism and (a) is established.

We now prove (b). Part (a) yields

$$
(A: I)=(I \cap J): I=J:(I+J) .
$$

It follows that, if $(A: I) \neq J$, then $(I+J) \subseteq P$ for some prime ideal $P$ of $R$ with $P \in \operatorname{Ass}(R / J)$. This is a contradiction because every associated prime of $R / J$ has grade $f$ but grade $(I+J) \geq f+1$. 


\section{Section 9. Properties of the Rings $R / J$ in the generic Case.}

Now that we have done the hard work of proving that the ideal $J(\rho)$ is perfect, when $\rho$ is a generic almost alternating matrix, we are able to harvest a great many results by using standard, well-known, techniques. The main result in this section is Theorem 9.13. The standing hypotheses are

$R_{0}$ is a commutative noetherian ring; $\rho=\left[\begin{array}{ll}X & Y\end{array}\right]$ is a generic almost alternating map of shape $(g, f)$ over the polynomial ring $R=R_{0}[X, Y]$; the complexes $\mathfrak{D}^{q}=\mathfrak{D}^{q}(\rho)$ are defined in section 2 ; the ideals $J=J(\rho)$ and $I=I(\rho)$ are defined in $(4.10) ; \bar{R}=R / J$; and $\bar{I}$ is the ideal $(I+J) / J$ of $\bar{R}$.

In Theorem 9.13 we calculate the divisor class group of $\bar{R}$, provided $R_{0}$ is a normal domain. Suppose, for the time being, that $g$ is odd, $f \geq 3$, and $R_{0}$ is a local, Cohen-Macaulay, factorial domain which satisfies the Serre condition $\left(R_{f+1}\right)$. Let $S$ be the complement of the irrelevant maximal ideal in $R_{0}[X]$. The class group of $S^{-1}(\bar{R})$ has been calculated by Huneke and Ulrich [34, Theorem 3.4] as part of their study of generic residual intersections of strongly Cohen-Macaulay ideals. Our ideal $I$ is not an arbitrary strongly Cohen-Macaulay ideal, but is, in fact, a very specific strongly Cohen-Macaulay ideal; and therefore, we have been able to combine the techniques of [34] with the techniques of Bruns [6] (for determinantal ideals) in order to prove a result analogous to [34, Theorem 3.4], but with far fewer hypotheses on the ring $R_{0}$. The ring $R_{0}$ must be a normal domain in order to study $\mathrm{C} \ell\left(R_{0}\right)$. In Theorem 9.13 we have imposed no other hypothesis on $R_{0}$. Furthermore, in Theorem 9.13 we are able to consider two cases not found in [34, Theorem 3.4]. The case when $g$ is even corresponds to residual intersections which are not completely generic. The case $f=2$ has nothing at all to do with residual intersections.

Our first result concerns the $\bar{R}$-modules $H_{0}\left(\mathfrak{D}^{q}\right)$. Recall, from Theorem 7.3 (e), that $H_{0}\left(\mathfrak{D}^{q}\right) \cong \bar{I}^{q}$ for all $q \geq 0$ (except in the case that $f=0$ and $g$ is even). In the proof of Theorem $7.3(\mathrm{~d})$ we applied Proposition 1.25 and showed that the $\bar{R}$-module $H_{0}\left(\mathfrak{D}^{q}\right)$ satisfies the Serre-like condition $\left(\widetilde{S}_{1}\right)$ for all $q \geq-1$. We are able to improve this conclusion, for most $f$, by making better use of the estimate of the grade of the ideals $I_{t}(\rho)$ which is given in Observation 5.13. Our chief reason for wanting to improve the conclusion $\left(\widetilde{S}_{1}\right)$ is, of course, due to the fact that if $\bar{R}$ is a normal domain and $M$ is an $\bar{R}$-module which satisfies the condition $\left(\widetilde{S}_{2}\right)$, then $M$ is a reflexive $\bar{R}-$ module.

Proposition 9.2. If the notation of (9.1) is adopted, then the $\bar{R}$-module $H_{0}\left(\mathfrak{D}^{q}\right)$ satisfies the condition

$$
\begin{cases}\left(\widetilde{S}_{1}\right) & \text { if } f=0 \text { and } g \text { is odd } \\ \left(\widetilde{S}_{3}\right) & \text { if } f=0 \text { and } g \text { is even } \\ \left(\widetilde{S}_{f}\right) & \text { if } f \geq 1\end{cases}
$$

for all $q \geq-1$. In particular, if $f \geq 2, g \geq 2, q \geq 1$, and $P \in \operatorname{Spec} R$ with $P \in \operatorname{Ass}\left(\bar{R} / \bar{I}^{q}\right)$, then depth $\bar{R}_{P}=1$ and grade $P=\operatorname{depth} R_{P}=f+1$. 
Note. If $-1 \leq q \leq f-1$, then Corollary 1.26 shows that the $\bar{R}$-module $H_{0}\left(\mathfrak{D}^{q}\right)$ satisfies $\left(\widetilde{S}_{n}\right)$ for all $n$. The present result is significant because it holds for all $q$ which are at least -1 .

Proof. There is nothing more to prove if $f=0$ and $g$ is odd. For the other two cases we define the ideals $F_{i}$ exactly as in the proof of Theorem 7.3 (d). Suppose that $f=0$ and $g$ is even. Let $i$ be an even integer with $2 \leq i \leq g-2$. Recall from (6.19) that

$$
\operatorname{Pf}_{g-i}(\rho) \subseteq F_{i} \subseteq F_{i+1} .
$$

It follows from (1.24) that

$$
\text { grade } F_{i}-i \geq \operatorname{grade} \operatorname{Pf}_{g-i}(\rho)-i=\frac{(i+2)(i+1)}{2}-i \geq 4 ;
$$

and therefore, grade $F_{j} \geq j+3$ for all $j$ with

$$
\text { grade } J+1=2 \leq j \leq g-1 .
$$

The proof of this case is completed by appealing to Proposition 1.25.

Now we assume that $f \geq 1$. Line (7.7) yields that $I_{e-i}(\rho) \subseteq F_{i}$ for all $i$ with

$$
f+1 \leq i \leq e-1
$$

and therefore, Observation 5.13 shows that

grade $F_{i} \geq \operatorname{grade} I_{e-i}(\rho) \geq \frac{(i-f+1)(i-f)}{2}+(i-f+1) f \geq(i-f)+2 f=i+f$.

Once again, Proposition 1.25 completes the proof.

Finally, we consider the last assertion. The fact that the $\bar{R}$-module $\bar{I}^{q} \cong$ $H_{0}\left(\mathfrak{D}^{q}\right)$ satisfies $\left(\widetilde{S}_{2}\right)$ guarantees that depth $\bar{R}_{P} \leq 1$. On the other hand, the ideal $J R_{P}$ of $R_{P}$ is perfect of grade $f$, so

$$
1 \geq \operatorname{depth} \bar{R}_{P}=\operatorname{grade} \frac{P R_{P}}{J R_{P}} \geq \operatorname{grade} P R_{P}-\operatorname{grade} J R_{P}=\operatorname{depth} R_{P}-f ;
$$

therefore, Proposition 5.14 yields that

$$
f+1 \geq \operatorname{depth} R_{P} \geq \operatorname{grade} P \geq f+1,
$$

and equality holds everywhere.

Example 9.3. The conclusions of Proposition 9.2 are optimal. If $f=0$ and $g \geq 3$ is odd, then $H_{0}\left(\mathfrak{D}^{1}\right)=I$ is a grade three ideal of the ring $\bar{R}=R$; thus, $I$ is not reflexive and does not satisfy $\left(\widetilde{S}_{2}\right)$. Furthermore, if $f \geq 1, g=2$, and $R_{0}$ is a field $k$, then $N=H_{0}\left(\mathfrak{D}^{f}\right)$ does not satisfy $\left(\widetilde{S}_{f+1}\right)$. In this case, $R$ is the polynomial ring $k[x, Y]$ where $x$ is a single indeterminate and $Y_{2 \times f}$ is a generic matrix. Let $P$ be the irrelevant maximal ideal of $R$. We saw in Example 4.12 (b) that $J$ is the grade $f$ ideal $(x)+I_{2}(Y)$ of $R$. Observe that depth $R_{P}=2 f+1$ and $\operatorname{depth} \bar{R}_{P}=f+1$. The projective dimension of the $R_{P}$-module $N_{P}$ is $f+1$; thus, depth $N_{P}=f$ and we see that $N$ does not satisfy $\left(\widetilde{S}_{f+1}\right)$. We emphasize that if $f=1$ and $g=2$, then the ideal $I \bar{R}$, which is $H_{0}\left(\mathfrak{D}^{f}\right)$, is not reflexive. Indeed, $I \bar{R}$ is the grade two ideal $\left(y_{11}, y_{21}, x\right) /(x)$ of the ring $\bar{R}=R_{0}\left[y_{11}, y_{21}, x\right] /(x)$. 
Theorem 9.4. Adopt the notation of (9.1).

(a) If $R_{0}$ is a domain, then so is $\bar{R}$.

(b) Let $k$ be an integer such that

$$
\begin{cases}k<\infty & \text { if } f=0 \text { and } g \text { is odd, } \\ k \leq f & \text { if } 2 \leq f \\ k \leq 4 & \text { if either } f=1, \text { or else } f=0 \text { and } g \text { is even }\end{cases}
$$

(i) If $R_{0}$ satisfies the Serre condition $\left(S_{k+1}\right)$, then so does $\bar{R}$.

(ii) If $R_{0}$ satisfies the Serre conditions $\left(R_{k}\right)$ and $\left(S_{k+1}\right)$, then so does $\bar{R}$. In particular, if the ring $R_{0}$ is reduced, then so is $\bar{R}$; if the ring $R_{0}$ is normal, then so is $\bar{R}$.

Proof.

(a) One could prove most of this result by maneuvering into the situation of generic residual intersection and then appealing to [32, Theorem 3.3]. The proof we give is identical to the proof in [9, Theorem 2.10] that generic determinantal ideals are prime. We proceed by induction on $g$. If $g \leq 2$, then the ideal $J$ has been studied previously (see Example 4.12) and the result is known. Henceforth, we assume that $g \geq 3$. Let $P$ be a prime ideal of $R$ with $P \in \operatorname{Ass} \bar{R}$. Since the ideal $J$ is perfect of grade $f$ or $f+1$, we see that

$$
\text { grade } I_{1}(\rho)=\left(\begin{array}{l}
g \\
2
\end{array}\right)+f g>f+1 \geq \operatorname{grade} J=\text { grade } P .
$$

It follows that some entry $z$ from $\rho$ is not in $P$. Lemma 5.6 and the note following it show that $J\left(\rho \otimes R\left[z^{-1}\right]\right)=J\left(\rho^{\prime}\right)$ for some generic almost alternating map $\rho^{\prime}$ of shape $\left(g^{\prime}, f\right)$ where $g^{\prime}$ is equal to either $g-1$ or $g-2$. The induction hypothesis ensures that $\bar{R}\left[z^{-1}\right]$ is a domain. It suffices to prove that $z$ is a non-zero-divisor on $\bar{R}$. Observe that $P$ is the only associated prime of $\bar{R}$ which does not contain $z$. If $z \in Q \in \operatorname{Ass} \bar{R}$, then there is an entry $w$ of $\rho$ with $w \notin Q$. We repeat the above reasoning to see that $w$ must be in $P$. We have reached a contradiction because $P \bar{R}\left[z^{-1}\right]=0$, but Lemma 9.6 shows that the image of $w$ in $\bar{R}\left[z^{-1}\right]$ is not zero. Therefore $P$ is the only associated prime of $\bar{R}$, and $z$ is regular on $\bar{R}$. It follows that $\bar{R}$ is a domain since $\bar{R}\left[z^{-1}\right]$ has this property.

(b) If $f=0$ and $g$ is odd, then $\bar{R}=R$ and the assertion is obvious. We next assume $f \geq 2$. Let $P$ be a prime ideal of $R$ with $J \subseteq P$ and $\operatorname{depth} \bar{R}_{P} \leq k$. Define $A$ to be the ring

$$
A=\left(R_{0}\right)_{P \cap R_{0}} .
$$

For (i) it suffices to show that $\bar{R}_{P}$ is Cohen-Macaulay; for (ii) it suffices to show that $\bar{R}_{P}$ is regular. Since $J R_{P}$ is a perfect ideal of grade $f$ in the ring $R_{P}$, we see that

grade $P R_{P}-f=\operatorname{grade} P R_{P}-\operatorname{grade} J R_{P} \leq \operatorname{grade} \frac{P R_{P}}{J R_{P}}=\operatorname{depth} \bar{R}_{P} \leq k ;$ 
and therefore, it follows, from Observation 5.13, that

$$
\text { grade } P \leq \operatorname{grade} P R_{P} \leq f+k \leq 2 f<2 f+1 \leq \operatorname{grade} I_{g-1}(\rho)
$$

Apply Lemma 5.6 and the note following it. There is a polynomial ring $\mathcal{R}=$ $R_{0}\left[z_{1}, \ldots, z_{n}\right]$ contained in $R$, and an element $\Delta \in \mathcal{R}$ with $\Delta \notin P$, and a generic almost alternating map $\rho^{\prime}=\left[0 Y^{\prime}\right]$ of shape $(1, f)$ over $\mathcal{R}\left[\Delta^{-1}, Y^{\prime}\right]=R\left[\Delta^{-1}\right]$, such that $J R\left[\Delta^{-1}\right]=J\left(\rho^{\prime}\right)$. We know from Example 4.12 (a) that $J\left(\rho^{\prime}\right)=I_{1}\left(Y^{\prime}\right)$. It is now easy to verify that

$$
\left(P \cap \mathcal{R}, I_{1}\left(Y^{\prime}\right)\right) \mathcal{R}\left[Y^{\prime}, \Delta^{-1}\right]=P R\left[\Delta^{-1}\right]
$$

and therefore,

$$
R_{P}=R\left[\Delta^{-1}\right]_{P R\left[\Delta^{-1}\right]}=\mathcal{R}\left[\Delta^{-1}, Y^{\prime}\right]_{\left(P \cap \mathcal{R}, Y^{\prime}\right) \mathcal{R}\left[\Delta^{-1}, Y^{\prime}\right]}=\mathcal{R}\left[Y^{\prime}\right]_{\left(P \cap \mathcal{R}, Y^{\prime}\right)}
$$

It follows that $\bar{R}_{P}=\mathcal{R}_{P \cap \mathcal{R}}$ and that

$$
\operatorname{depth} A \leq \operatorname{depth} \mathcal{R}_{P \cap \mathcal{R}}=\operatorname{depth} \bar{R}_{P} \leq k \text {. }
$$

The hypothesis guarantees that $A$ is Cohen-Macaulay in case (i) and that $A$ is regular in case (ii). The ring $\mathcal{R}_{P \cap \mathcal{R}}=\bar{R}_{P}$ is a localization of a polynomial ring over $A$ and therefore, it also has the desired property.

Finally, we suppose that, either, $f=1$; or else, $f=0$ and $g$ is even. We saw, in Example 4.12 (e), that, in each of these cases, $J$ is a principal ideal generated by the pfaffian of a generic alternating $n \times n$ matrix " $T$ ". In this situation, $J R_{P}$ is a perfect ideal of grade one and (9.5) becomes

$$
\text { grade } P \leq \text { grade } P R_{P} \leq 1+k \leq 5<6 \leq \operatorname{grade} \mathrm{Pf}_{n-2}(T)
$$

The only other change in the rest of the proof is that $\rho^{\prime}$ will have shape $(2,0),(2,1)$, or $(1,1)$. In any event, $J\left(\rho^{\prime}\right)$ is generated by a single indeterminate.

Lemma 9.6. In the notation of (9.1) assume that $g \geq 3$. If $z$ and $w$ are entries of $\rho$, then $z w$ is not in the radical of $J$.

Proof. The $R$-ideals $J$ and $I_{g}(\rho)$ have the same radical by Corollary 5.5; consequently, it suffices to exhibit an $R_{0}$-algebra homomorphism $\alpha: R \rightarrow R_{0}$ such that $\alpha(z w)$ is not an element of $\alpha\left(\operatorname{rad} I_{g}(\rho)\right)$. Let $\alpha(z)=\alpha(w)=1$. It is possible to define the rest of $\alpha$ so that $I_{g}(\alpha \rho)=0$. For example, if $\rho$ has shape $(3,1)$ with $z=x_{23}$ and $w=y_{11}$, then define $\alpha$ so that

$$
\alpha(\rho)=\left[\begin{array}{rrr|r}
0 & 0 & 1 & 1 \\
0 & 0 & 1 & 1 \\
-1 & -1 & 0 & 0
\end{array}\right] .
$$

The only other interesting case is

$$
\rho=\left[\begin{array}{cccc}
0 & z & x_{13} & x_{14} \\
-z & 0 & x_{23} & x_{24} \\
-x_{13} & -x_{23} & 0 & w \\
-x_{14} & -x_{24} & -w & 0
\end{array}\right]
$$


In this case one can let $\alpha\left(x_{13}\right)=\alpha\left(x_{24}\right)=1$ and $\alpha\left(x_{23}\right)=\alpha\left(x_{14}\right)=0$.

Remark 9.7. Let $R_{0}$ be a commutative noetherian ring and $X$ be a generic $g \times g$ alternating matrix with $g$ odd. We know from (1.24) or [14] that the $R_{0}[X]$-ideal $\mathrm{Pf}_{g-1}(X)$ is perfect of grade three. Buchsbaum and Eisenbud [14, Proposition 6.1] proved that if $R_{0}$ is a field, then $\operatorname{Pf}_{g-1}(X)$ is a prime ideal. One can use the proof employed in Theorem 9.4 to obtain the same conclusion whenever $R_{0}$ is a domain.

Example 9.8. In general, the ring $\bar{R}$ will not be any more regular than the bounds guaranteed by Theorem 9.4 (b). Let $R_{0}$ be a field $k$ and let $P$ be the irrelevant maximal ideal of $R$. If $\rho$ has shape $(4,0)$, then $\bar{R}=k[X] /(\operatorname{pf}(X))$, where $X$ is a generic $4 \times 4$ alternating matrix. Observe that $\bar{R}_{P}$ has dimension five but is not regular. If $\rho$ has shape $(2, f)$ and $f \geq 2$, then $\bar{R}=k[x, Y] /\left(x, I_{2}(Y)\right)$, where $x$ is an indeterminate and $Y$ is a generic $2 \times f$ matrix. We see that $\bar{R}_{P}$ has dimension $f+1$, but is not regular.

Remarks 9.9. In the notation of (9.1), let $R_{0}$ be regular local and let $\mathfrak{m}$ be the irrelevant maximal ideal of $R$.

(a) One consequence of Theorem 9.4 (b) is that if $f \geq 4$ and $g \geq 2$, then the ideal $J_{\mathfrak{m}}$ is not in the linkage class of a complete intersection. Indeed, Huneke and Ulrich [31, Theorem 4.2] have proved that if $I$ is a licci ideal in a regular local ring $S$ and $S / I$ is not a Gorenstein ring, then no deformation of $S / I$ satisfies the condition $\left(R_{4}\right)$. A quick look at Observation 4.28 shows that $(R / J)_{\mathfrak{m}}$ is not Gorenstein because the resolution $\mathfrak{D}_{\mathfrak{m}}^{0}$ is minimal and the last Betti number is $\left(\begin{array}{c}f+g-3 \\ f-2\end{array}\right)$, which is larger than one.

(b) If $g$ is odd and $f \geq 3$, then Huneke and Ulrich [34, Theorem 2.4] predict that $(R / J)_{\mathfrak{m}}$ satisfies the Serre condition $\left(R_{k}\right)$ for $k=\min \{5, f\}$. In Theorem 9.4 (b.ii) we do not require $k \leq 5$.

If $R_{0}$ is a local Cohen-Macaulay domain and $g$ is odd, then most of the next result could be deduced from [34, Theorem 3.4].

Lemma 9.10. Adopt the notation of (9.1). Assume that $f \geq 2, g \geq 2$, and that $R_{0}$ is a domain. If $R_{0}$ satisfies the Serre condition $\left(S_{2}\right)$, then the ideal $\bar{I}$ of $\bar{R}$ is prime of height one, and for all $q \geq 1$, the symbolic $q^{\text {th }}$ power of $\bar{I}, \bar{I}^{(q)}$, is equal to the ordinary $q^{\text {th }}$ power of $\bar{I}, \bar{I}^{q}$.

Proof. Let $P$ be a prime ideal of $R$ with $P \in$ Ass $\left(\bar{R} / \bar{I}^{q}\right)$. We know from Proposition 9.2 that depth $\bar{R}_{P}=1$ and grade $P=f+1$. The ring $\bar{R}$ satisfies the Serre condition $\left(S_{2}\right)$ by Theorem $9.4(\mathrm{~b})$; and therefore, ht $P \bar{R}=1$ and $\bar{I}^{q}$ is an unmixed ideal of height one. The claim about the symbolic powers $\bar{I}^{(q)}$ follows as soon as we prove that $\bar{I}$ is prime and our proof of this fact is modeled after the proof of Theorem 9.4 (a).

We proceed by induction on $g \geq 2$. If $g=2$, then $I+J$ is the prime ideal

$$
\left(x, y_{11}, y_{21}\right)+I_{2}\left(Y^{\prime}\right)
$$

where $Y^{\prime}$ is the matrix formed by deleting the first column of $Y$. If $g=3$, then $I+J$ is the prime ideal $I_{1}(X)+I_{3}(Y)$. So, let $g \geq 4$; then Proposition 5.14 assures 
us that $\operatorname{Pf}_{2}(X)$ is not contained in $P$. Suppose that $\Delta=x_{i j} \notin P$. By Lemma 5.6 there is a generic almost alternating matrix $\rho^{\prime}=\left[X^{\prime} Y^{\prime}\right]$ of shape $(g-2, f)$ with $J_{\Delta}=J\left(\rho^{\prime}\right)$ and $I_{\Delta}=I\left(\rho^{\prime}\right)$. It follows, from the induction hypothesis, that $(I+J)_{\Delta}$ is a prime ideal in $R_{\Delta}$. We finish by imitating the proof of Theorem 9.4 (a). Let $\Delta^{\prime}=x_{k \ell}$ be some other entry of $X$ with $\Delta^{\prime} \notin(I+J)$. If suffices to show that $\left(\Delta \Delta^{\prime}\right)^{n} \notin(I+J)$ for any positive integer $n$. Since $g \geq 4$, one can use the trick of Lemma 9.6 to establish this fact.

Like so much of this section, the idea to make use of the next result in the proof of Theorem 9.13 was inspired by [34]. This time the inspiration came from [34, Lemma 3.3]. The result has been taken from the theory of linkage [30, Propositions 2.5 and 2.6]; however linkage theory always assumes that the ideals in question are perfect or that the ring in question is Gorenstein local, or at least CohenMacaulay local. In fact, one is able to squeeze out some of the same conclusions with less hypotheses.

Lemma 9.11. Let $A$ be a commutative noetherian ring which satisfies the Serre condition $\left(S_{2}\right)$ and let $L$ be a height one ideal in $A$. Let $f_{1}, \ldots, f_{n}$ be a generating set for $L ; T_{1}, \ldots, T_{n}$ be indeterminates over $A ; B$ be the polynomial ring $A\left[T_{1}, \ldots, T_{n}\right]$; $x$ be the element $\sum f_{i} T_{i}$ of $B$, and $K$ be the $B$-ideal $((x): L B)$. If $(L)_{\mathbf{q}}$ is a principal ideal for all height one prime ideals $\mathbf{q}$ of $A$ which contain $L$, then the following statements hold.

(a) The $B$-ideal $K$ is unmixed of height one.

(b) The intersection $K \cap L B$ is equal to $(x)$.

(c) If $A$ is a domain, then $K$ is a height one prime ideal.

Proof.

(a) Let $P$ be a prime ideal of $B$ with $P \in \operatorname{Ass}(B / K)$. There is an element $b \in B$ with $b \notin K$ such that $P b \subseteq K$. It follows that $P b L \subseteq K L \subseteq(x)$, but that $b L$ is not contained in $(x)$. Thus, there is a prime ideal $Q$ of $B$ with $K \subseteq P \subseteq Q \in$ Ass $(B /(x))$. The ideal $L$ of $A$ has grade one because $A$ satisfies the Serre condition $\left(S_{2}\right)$; therefore, the element $x$ of $B$ is regular. Furthermore, the $\operatorname{ring} B$ satisfies the Serre condition $\left(S_{2}\right)$; so

$$
\text { ht } Q=1 \quad \text { for all } \quad Q \in \operatorname{Ass}\left(\frac{B}{(x)}\right)
$$

We see that $1 \leq \mathrm{ht}(K) \leq \mathrm{ht}(P) \leq \mathrm{ht}(Q)=1$; and (a) is established.

(b) Let $Q$ be an arbitrary element of $\operatorname{Ass}(B /(x))$. It suffices to show that $(K \cap$ $L B)_{Q}$ is contained in $(x)_{Q}$. If $L B$ is not contained in $Q$, then the definition of $K$ shows us that $K_{Q}=(x)_{Q}$. Suppose that $L B \subseteq Q$. We show that $(L B)_{Q}=(x)_{Q}$. Let $\mathbf{q}=Q \cap A$. It follows from (9.12) that the height of $\mathbf{q}$ is one and that $Q=\mathfrak{q} B$. One of the $f$ 's, say $f_{1}$, generates $(L)_{\mathfrak{q}}$. It follows that there is an element $s$ in $A$ with $s \notin \mathbf{q}$ and there are elements $a_{2}, \ldots, a_{n}$ in $A$ with $s f_{i}=a_{i} f_{1}$ in $A$ for all $i$. We see that $s x=b f_{1}$ where $b$ is the element $s T_{1}+a_{2} T_{2}+\cdots+a_{n} T_{n}$ of $B$. Assertion (b) is established because $b \notin Q$.

(c) One consequence of the proof of (b) is that $L$ is not contained in any associated prime of $B / K$. A prime avoidance argument yields an element $a$ in $\left(f_{2}, \ldots, f_{n}\right) A$ 
with $s=\left(f_{1}+a\right)$ regular on $B / K$. The $B$-ideal $K$ is prime because $K_{s}=\left(x_{s}\right)$ and $x_{s}$ is an indeterminate over the domain $B_{s}$.

We define one more ideal, $\overline{J^{\prime}}$, of $\bar{R}$. In the notation of (9.1) with $g \geq 2$, let $X^{\prime}$ be the matrix obtained by deleting row and column $g$ from $X, Y^{\prime}$ the matrix obtained by deleting row $g$ from $Y, \rho^{\prime}$ the generic almost alternating matrix $\left[X^{\prime} Y^{\prime}\right]$ of shape $(g-1, f), J^{\prime}$ the ideal $J\left(\rho^{\prime}\right)$ of $R$, and $\overline{J^{\prime}}$ the ideal $\left(J^{\prime}+J\right) / J$ of $\bar{R}$. If $g=1$, then let $\overline{J^{\prime}}$ be the entire ring $\bar{R}$. If $R$ is a Cohen-Macaulay domain which is a factor ring of a local Gorenstein ring, $g$ is odd, and $f \geq 3$, then the next theorem also follows from [34, Theorems 3.4 and 3.5].

Theorem 9.13. Adopt the notation of (9.1).

(a) If either, $f \geq 1$, or else, $g$ is odd, then the following statements hold.

(i) The $\bar{R}$-module $H_{0}\left(\mathfrak{D}^{1}\right)$ is isomorphic to the $\bar{R}$-ideal $\bar{I}$.

(ii) For each $q \geq 1$, the $\bar{R}$-module $H_{0}\left(\mathfrak{D}^{q}\right)$ is isomorphic to both the $q^{\text {th }}$ symmetric power of $\bar{I}$ and the ordinary $q^{\text {th }}$ power of $\bar{I}$ :

$$
H_{0}\left(\mathfrak{D}^{q}\right) \cong S_{q}(\bar{I}) \cong \bar{I}^{q}
$$

(iii) The $\bar{R}$-module $H_{0}\left(\mathfrak{D}^{-1}\right)$ is isomorphic to the ideal $\overline{J^{\prime}}$ of $\bar{R}$.

(b) If $R_{0}$ is a normal domain, then $\bar{R}$ is also a normal domain and the following statements hold.

(i) If, either, $f \leq 1$, or else, $g=1$, then $\mathrm{C} \ell(\bar{R}) \cong \mathrm{C} \ell\left(R_{0}\right)$.

(ii) If $f \geq 2$ and $g \geq 2$, then the following statements hold.

$(\alpha)$ The $\bar{R}$-ideals $\overline{J^{\prime}}$ and $\bar{I}^{q}$ are divisorial for all $q \geq 1$.

( $\beta)$ The divisor class group of $\bar{R}$ is isomorphic to $\mathrm{C} \ell\left(R_{0}\right) \oplus \mathbb{Z}$.

$(\gamma)$ The summand $\mathbb{Z}$ in $\mathrm{C} \ell(\bar{R})$ is generated by $[\bar{I}]$.

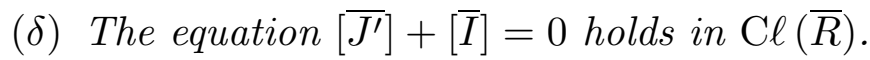

( $\varepsilon)$ Let $M$ be a reflexive $\bar{R}$-module of rank one with $[M]=q[\bar{I}]$ in $\mathrm{C} \ell(\bar{R})$ for some integer $q$. If either, $R_{0}$ is a Cohen-Macaulay ring and $-1 \leq q$, or else, $R_{0}$ is a Gorenstein ring and $q$ is any integer, then $M$ is a Cohen-Macaulay $\bar{R}$-module if and only if $-1 \leq q \leq f-1$.

Proof. Recall, from Example 4.8, that if $\rho$ is a generic almost alternating map of shape $(1, f)$, then $H_{0}\left(\mathfrak{D}^{q}\right)=\bar{R}=R_{0}$ for all integers $q$. The $\bar{R}$-ideals $\bar{I}$ and $\overline{J^{\prime}}$ are both equal to $\bar{R}$; hence, the theorem holds in this case, but is not informative. Henceforth, we assume that $g \geq 2$.

(a) Assertions (i) and (ii) are proved in Theorem 7.3 (e) and Proposition 4.13 (b). We now prove (iii). The $\bar{R}$-module $H_{0}\left(\mathfrak{D}^{-1}\right)$ is torsion-free of rank one by Theorem $7.3(\mathrm{~d})$. Recall the ideal $\widetilde{J}_{\gamma}$ of $R$ from (4.19). If $\gamma$ is set equal to the basis element $\gamma_{g}$, then it is easy to see that $\left(\widetilde{J}_{\gamma}+J\right)=\left(J^{\prime}+J\right)$. It follows, from Proposition 4.13 (e), that there is an $\bar{R}$-module surjection $H_{0}\left(\mathfrak{D}^{-1}\right) \rightarrow \overline{J^{\prime}}$. Recall, also, from Proposition 5.19, that the grade of the $R$-ideal $J^{\prime}+J$ is at least $f+1$. The $R$-ideal $J$ is perfect of grade $f$, so the $\bar{R}$-ideal $\overline{J^{\prime}}$ has positive grade. The conclusion follows from Observation 1.27. 
(b) Proposition 9.4 shows that $\bar{R}$ is a normal domain. We first consider assertion (i). If $f=0$ and $g$ is odd, then $J$ is the zero ideal and $\bar{R}=R$ is a polynomial ring over $R_{0}$; therefore, $\mathrm{C} \ell(\bar{R})=\mathrm{C} \ell\left(R_{0}\right)$. If, either, $f=1$, or else, $f=0$ and $g$ is even, then Example 4.12 (e) shows that $\bar{R}$ is a polynomial ring over $R_{0}[T] /\left(\operatorname{Pf}_{n}(T)\right)$, where $T$ is a generic alternating $n \times n$ matrix and $n$ is even. Now, [2] implies that $\mathrm{C} \ell(\bar{R}) \cong \mathrm{C} \ell\left(R_{0}\right)$.

(b.ii) Assertion $(\alpha)$ is proved in Proposition 9.2. The proof of $(\beta)$ and $(\gamma)$ is next. We know from Lemma 9.10 that the ideal $\bar{I}$ of $\bar{R}$ is prime of height one. Let $\widetilde{X}_{\langle 1\rangle}, \ldots, \widetilde{X}_{\langle g\rangle}$ be the elements of $R$ which are given in (4.16). The image of these elements in $\bar{R}$ is a generating set for $\bar{I}$. Let

$$
T=\left[\begin{array}{c}
T_{1} \\
\vdots \\
T_{g}
\end{array}\right]
$$

be a matrix of indeterminates, $v$ one more indeterminate, $w$ the element $\sum T_{i} \widetilde{X}_{\langle i\rangle}$ of $R[T, v]$, and $x$ the image of $w$ in $\bar{R}[T, v]$. Define $K$ to be the $\bar{R}[T, v]-$ ideal

$$
K=(x): \bar{I} \bar{R}[T, v] .
$$

The ring $\bar{R}$ is a normal domain, so we may apply Lemma 9.11 in order to see that:

(a) $K$ is a height one prime ideal of $\bar{R}[T, v]$,

(b) $K$ and $\bar{I} \bar{R}[T, v]$ are the only height one prime ideals of $\bar{R}[T, v]$ which contain the element $x$, and

(c) $[K]+[\bar{I} \bar{R}[T, v]]=0$ in $\mathrm{C} \ell(\bar{R}[T, v])$.

Let $Q$ be the ring $\bar{R}\left[T, v, T_{1}^{-1}\right]$. Nagata's Theorem [21, Section 7], shows that the natural map $\mathrm{C} \ell(Q) \rightarrow \mathrm{C} \ell\left(Q_{x}\right)$ is a surjection whose kernel is generated by the classes of those height one prime ideals of $Q$ which contain $x$; thus, we have a short exact sequence

$$
0 \rightarrow \mathbb{Z}[\bar{I} Q]+\mathbb{Z}[K Q] \rightarrow \mathrm{C} \ell(Q) \rightarrow \mathrm{C} \ell\left(Q_{x}\right) \rightarrow 0 .
$$

On the other hand, the map $R_{0} \rightarrow \bar{R}$ is flat because of the theory of generic perfection (see [9, Proposition 3.2]); so there are natural maps

$$
\mathrm{C} \ell\left(R_{0}\right) \rightarrow \mathrm{C} \ell(\bar{R}) \stackrel{\cong}{=} \mathrm{C} \ell(Q) .
$$

In Lemma 9.20 we prove that the natural map

$$
\mathrm{C} \ell\left(R_{0}\right) \rightarrow \mathrm{C} \ell\left(Q_{x}\right)
$$

is an isomorphism. It follows that the short exact sequence of (9.16) is split; hence, $\mathrm{C} \ell(Q) \cong\left(\mathrm{C} \ell\left(R_{0}\right) \oplus \mathbb{Z}[\bar{I} Q]\right) ;$ and therefore

$$
\mathrm{C} \ell(\bar{R}) \cong \mathrm{C} \ell\left(R_{0}\right) \oplus \mathbb{Z}[\bar{I}] .
$$


It is clear that $\bar{I}$ has infinite order in $\mathrm{C} \ell(\bar{R})$ because $\bar{I}^{q}$ is a representative of $q[\bar{I}]$ for all $q \geq 1$ and we know that $\bar{I}^{q}$ is not principal. (Indeed, $\mathfrak{D}^{q}$ is a graded resolution of $\bar{I}^{q}$. If $\mathfrak{m}$ is a maximal ideal of $R_{0}$ and $\mathfrak{M}$ is the maximal ideal $\mathfrak{m} R+(X, Y)$ of $R$, then $\mathfrak{D}_{\mathfrak{M}}^{q}$ is a minimal resolution of $\bar{I}^{q}$, but $\mathfrak{D}_{0}^{q}=K_{q 0}=S_{q}(G)$ which has rank greater than one because $g \geq 2$.)

(b.ii. $\delta)$ We know, from (9.15), that $[K]$ is the inverse of $[\bar{I} \bar{R}[T, v]]$ in $\mathrm{C} \ell(\bar{R}[T, v])$, where $K$ is defined in (9.14). Let $\widehat{K}$ be the pre-image of $K$ in $R[T, v]$; that is

$$
\widehat{K}=(w, J R[T, v]): I R[T, v] .
$$

Since $w \in I R[T, v]$, we see that

$$
\widehat{K}=(w,(J R[T, v]) \cap(I R[T, v])): I R[T, v] .
$$

The polynomial extension $R \rightarrow R[T, v]$ is flat so

$$
(J R[T, v]) \cap(I R[T, v])=(J \cap I) R[T, v] .
$$

We know from Corollary 8.9 that $(J \cap I)$ is the ideal $A(\rho)$ of Definition 4.10. Let $\widehat{\rho}$ be the generic almost alternating matrix $\left[\begin{array}{l|l}X \mid Y & T\end{array}\right]$ of shape $(g, f+1)$. Observe that $w$ is precisely equal to the element " $a_{f+1}$ " in Definition 4.10 formed from $\widehat{\rho}$. In other words,

$$
(w,(J R[T, v]) \cap(I R[T, v]))=A(\widehat{\rho}) .
$$

It is clear that $I R[T, v]=I(\widehat{\rho})$. Combine all of these observations in order to obtain $\widehat{K}=(A(\widehat{\rho}): I(\widehat{\rho}))$. We use Corollary 8.9, again, in order to see that the $R[T, v]$-ideals $\widehat{K}$ and $J(\widehat{\rho})$ are equal. We know from (7.9) that $H_{0}\left(\mathfrak{D}^{-1} \otimes_{R} R[T, v]\right)$ is isomorphic to the ideal $\widehat{J} / J$ of $\bar{R}[T, v]$. On the other hand, (a.iii) shows that $H_{0}\left(\mathfrak{D}^{-1}\right)$ is isomorphic to the ideal $\overline{J^{\prime}}$ of $\bar{R}$. It follows that the extension of $\overline{J^{\prime}}$ to $\bar{R}[T, v]$ is isomorphic to $\widehat{J} / J$; and therefore, $\left[\overline{J^{\prime}}\right]+[\bar{I}]=0$ on $\mathrm{C} \ell(\bar{R})$.

(b.ii. $\varepsilon$ ) If $-1 \leq q \leq f-1$, then $M \cong H_{0}\left(\mathfrak{D}^{q}\right)$ is a perfect module over the CohenMacaulay ring $R$. It follows that $M$ is a Cohen-Macaulay $R$-module; and hence, a Cohen-Macaulay $\bar{R}$-module. Henceforth, we consider integers $q$ which are not in the range $-1 \leq q \leq f-1$. Let $\mathfrak{m}$ be a maximal ideal of $R_{0}$ and let $\mathfrak{M}$ be the maximal ideal $\mathfrak{m}+I_{1}(\rho)$ of $R$. We show that $M$ is not a Cohen-Macaulay $\bar{R}$-module by showing that $M_{\mathfrak{M}}$ is not a Cohen-Macaulay $\bar{R}_{\mathfrak{M}}$-module. If $f \leq q$, then $M \cong H_{0}\left(\mathfrak{D}^{q}\right)$ and it is clear from the length of the graded resolution $\mathfrak{D}^{q}$ that

$\operatorname{depth} M_{\mathfrak{M}}<\left[\operatorname{pd}_{R_{\mathfrak{M}}} M_{\mathfrak{M}}-\operatorname{pd}_{R_{\mathfrak{M}}} \bar{R}_{\mathfrak{M}}\right]+\operatorname{depth} M_{\mathfrak{M}}=\operatorname{depth} \bar{R}_{\mathfrak{M}}=\operatorname{dim} \bar{R}_{\mathfrak{M}}=\operatorname{dim} M_{\mathfrak{M}}$

The final part of the argument was taken from [34, Theorem 3.5]. The ring $R_{0}$ is Gorenstein and we write $\bar{R}$ and $M$ for $\bar{R}_{\mathfrak{M}}$ and $M_{\mathfrak{M}}$, respectively. Suppose that $M$ is Cohen-Macaulay for $[M]=q[\bar{I}]$ with $q \leq-2$. Then $\operatorname{Hom}_{\bar{R}}\left(\bar{I}^{-q}, \bar{R}\right)$ is CohenMacaulay. Recall, from Theorem 7.3 (c) and (e), that $\bar{I}^{f-2}$ is isomorphic to the canonical module $\operatorname{Ext}_{R}^{f}(\bar{R}, R)$ of $\bar{R}$. It follows that

$$
\operatorname{Hom}_{\bar{R}}\left(\bar{I}^{-q}, \bar{R}\right) \cong \operatorname{Hom}_{\bar{R}}\left(\bar{I}^{-q} \otimes_{\bar{R}} \bar{I}^{f-2}, \bar{I}^{f-2}\right) \cong \operatorname{Hom}_{\bar{R}}\left(\bar{I}^{f-2-q}, \bar{I}^{f-2}\right)
$$


is Cohen-Macaulay; and hence, $\bar{I}^{f-2-q}$ is Cohen-Macaulay because $\bar{I}^{f-2-q}$ is reflexive and $\bar{I}^{f-2}$ is a canonical module. But, $f-2-q \geq f$; and therefore, $\bar{I}^{f-2-q} \cong H_{0}\left(\mathfrak{D}^{f-2-q}\right)$ cannot be Cohen-Macaulay by the above.

Lemma 9.18. Let $R_{0}$ be a commutative noetherian domain, $X$ be a generic $g \times g$ alternating matrix for some integer $g$, and $R$ be the polynomial ring $R_{0}[X]$. If $x_{12}$ is the entry of $X$ in row 1 and column 2 and $X(12)$ is the pfaffian of the matrix obtained by deleting rows and columns 1 and 2 from $X$, then the $R$-ideals $\left(\operatorname{pf}(X), x_{12}\right)$ and $(\operatorname{pf}(X), X(12))$ are prime.

Proof. Let $X^{\prime}$ denote the submatrix

$$
X^{\prime}=\left[\begin{array}{lll}
x_{13} & \cdots & x_{1 g} \\
x_{23} & \cdots & x_{2 g}
\end{array}\right]
$$

of $X$. The proof is modeled on the proof of Theorem 9.4. We first consider the $R$-ideal $\left(\operatorname{pf}(X), x_{12}\right)$, which we denote $K$. If $g=2$, then the result is obvious. If $g=4$, then $K=\left(x_{12}, I_{2}\left(X^{\prime}\right)\right)$ is a hypersurface section of a determinantal ideal and is therefore prime. Henceforth, we assume that $g \geq 6$. The ideal $K$ is perfect of grade two because $(\operatorname{pf}(X))$ is a prime ideal of grade one by Theorem 9.4 (a) and $x_{12} \notin(\mathrm{pf}(X))$. Let $P$ be a prime ideal of $R$ with $P \in \operatorname{Ass}(R / K)$. The grade of $P$ is two and grade $I_{2}\left(X^{\prime}\right)=g-3>2$. So, some element $\Delta$ of $I_{2}\left(X^{\prime}\right)$ is not in $P$. Without loss of generality, we assume that

$$
\Delta=\operatorname{det}\left[\begin{array}{ll}
x_{1 g-1} & x_{1 g} \\
x_{2 g-1} & x_{2 g}
\end{array}\right] .
$$

We may not choose a basis so that $X_{\Delta}$ looks like

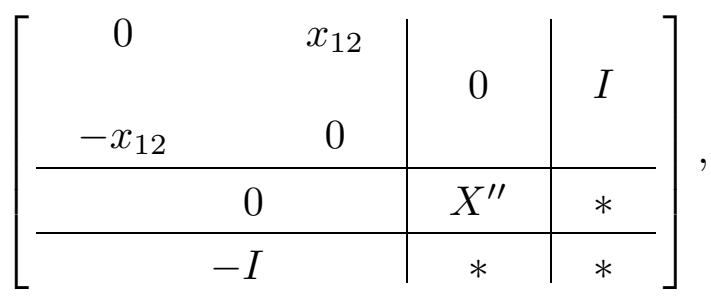

where $X^{\prime \prime}$ is a generic alternating $(g-4) \times(g-4)$ matrix. It follows that $K_{\Delta}$ is the prime ideal $\left(x_{12}, \operatorname{pf}\left(X^{\prime \prime}\right)\right)$. To finish the proof we need only see that $\Delta \Delta^{\prime}$ is not in the radical of $K$ for any other $2 \times 2$ minor $\Delta^{\prime}$ of $X^{\prime}$. Since $g \geq 6$, this fact can be established using the trick of Lemma 9.6. That is, one can choose values for the entries of $X$ so that $K=0$, but $\Delta \Delta^{\prime}=1$.

We now consider $K$ to be the ideal $(\operatorname{pf}(X), X(12))$. The proof uses induction on $g$, but otherwise is very similar. If $g=2$, then the assertion is meaningless. If $g=4$, then the result holds because of the previous case. Henceforth, we assume that $g \geq 6$. As before, some element $\Delta$ of $I_{2}\left(X^{\prime}\right)$ is not in the associated prime $P$, and we can write $X_{\Delta}$ in the form of (9.19). It follows that $K_{\Delta}=\left(X(12), \operatorname{pf}\left(X^{\prime \prime}\right)\right)$; and this ideal is prime by the induction hypothesis. The rest of the argument is unchanged. 
Lemma 9.20. In the notation of Theorem 9.13, the natural map $\mathrm{C} \ell\left(R_{0}\right) \rightarrow$ $\mathrm{C} \ell\left(Q_{x}\right)$ of $(9.17)$ is an isomorphism.

Proof. Let $Z$ be the generic alternating matrix

$$
Z=\left\{\begin{array}{l|c}
{\left[\begin{array}{c|c}
X & T \\
\hline-T^{\mathrm{t}} & 0
\end{array}\right]} & \text { if } g \text { is odd } \\
{\left[\begin{array}{c|cc}
X & Y_{* 1} & T \\
\hline-\left(Y_{* 1}\right)^{\mathrm{t}} & 0 & v \\
-T^{\mathrm{t}} & -v & 0
\end{array}\right] \quad \text { if } g \text { is even, }}
\end{array}\right.
$$

where, as always, $Y_{* j}$ is the $j^{\text {th }}$ column of $Y$. Let $z=\operatorname{pf}(Z)$. Recall that the element $w \in R[T]$, from the proof of Theorem 9.13 (b.ii), has the property that the image of $w$ in $\bar{R}[T]$ is equal to $x$. Observe that $z=w$ if $g$ is odd and $z=w+v \operatorname{pf}(X)$ if $g$ is even. Since $\operatorname{pf}(X) \in J$, we see that the image of $z$ in $Q$ is also $x$. Our proof consists of two parts: we show that there are indeterminates $U$ such that

$$
Q_{x}= \begin{cases}R_{0}\left[Z, T_{1}^{-1}\right]_{z}[U] & \text { if } g \text { is odd } \\ \left(\frac{R_{0}\left[Z, T_{1}^{-1}\right]_{z}}{(\operatorname{pf}(X))}\right)[U] & \text { if } g \text { is even, }\end{cases}
$$

and we show that $R_{0}$ and the rings on the right hand side of (9.21) have the same class group. Let us assume, for the time being, that (9.21) has been established. Suppose, first, that $g$ is odd. The elements $z$ and $T_{1}$ are prime in $R_{0}[Z]$, (use Theorem 9.4 (a) with $f=0$, if necessary), so it is clear that the natural map $\mathrm{C} \ell\left(R_{0}\right) \rightarrow \mathrm{C} \ell\left(Q_{x}\right)$ is an isomorphism. Now we assume that $g$ is even. It is clear that $T_{1}$ is a prime element in $R_{0}[Z] /(\operatorname{pf}(X))$. Lemma 9.18 shows that $z$ is a prime element in $R_{0}[Z] /(\operatorname{pf}(X))$. Thus, we have natural maps

$$
\mathrm{C} \ell\left(R_{0}\right) \rightarrow \mathrm{C} \ell\left(\frac{R_{0}[Z]}{(\operatorname{pf}(X))}\right) \stackrel{\cong}{\rightrightarrows} \ell\left(Q_{x}\right) .
$$

Theorem 9.13 (b.i) shows that the first map is also an isomorphism.

Lemma 5.6 establishes something similar to (9.21); unfortunately, though, the lemma is a little cavalier about what exactly has been inverted. Under the circumstances, it is easiest to establish (9.21) from scratch. Let $u_{i j}=y_{i j}-y_{1 j}\left(T_{j} / T_{1}\right)$. We first take $g$ to be odd. Let $U$ represent the set of indeterminates

$$
\{v\} \cup\left\{u_{i j} \mid 2 \leq i \leq g \quad \text { and } \quad 1 \leq j \leq f\right\} ;
$$

let $S$ be the ring $R_{0}\left[Z, U, T_{1}^{-1}\right]_{z}$; and for each $j$, with $1 \leq j \leq f$, let $a_{j}$ be the element of $J$ described in Definition 4.10 (b). Since

$$
Y_{* j}=\left(\frac{y_{1 j}}{T_{1}}\right) T+\left[\begin{array}{c}
0 \\
u_{2 j} \\
\vdots \\
u_{g j}
\end{array}\right],
$$


we see that

$$
a_{j}=\left(z / T_{1}\right) y_{1 j}+s_{j}
$$

for some element $s_{j}$ of $S$. It follows that $a_{1}, \ldots, a_{f}$ are algebraically independent over $S$ and $S\left[a_{1}, \ldots, a_{f}\right]=R\left[T, v, T_{1}^{-1}\right]_{z}$. The ideal $\left(a_{1}, \ldots, a_{f}\right)$ is a prime ideal of grade $f$ which is contained in the grade $f$ ideal $J R\left[T, v, T_{1}^{-1}\right]_{z}$. It follows that the two ideals are equal and $Q_{x}$, which is defined to be $(R / J)\left[T, v, T_{1}^{-1}\right]_{z}$, is equal to $S$.

Finally, we establish (9.21) for even $g$. The approach is similar to the above. Let $U$ represent the indeterminates

$$
\left\{u_{i j} \mid 2 \leq i \leq g \quad \text { and } \quad 2 \leq j \leq f\right\}
$$

let $S$ be the ring $R_{0}\left[Z, U, T_{1}^{-1}\right]_{z}$; and for each $j$, with $2 \leq j \leq f$, let $a_{j}$ be the element of $J$ described in Definition 4.10 (c). Equation (9.22) still holds; and therefore, $a_{2}, \ldots, a_{f}$ are algebraically independent over $S$ and $S\left[a_{2}, \ldots, a_{f}\right]=R\left[T, v, T_{1}^{-1}\right]_{z}$. The ideal $\left(\operatorname{pf}(X), a_{2}, \ldots, a_{f}\right)$ is a prime ideal of grade $f$ which is contained in the grade $f$ ideal $J R\left[T, T_{1}^{-1}\right]_{z}$. The two ideals are equal and $Q_{x}=S /(\operatorname{pf}(X))$. 


\section{SECTION 10. THE RESIDUAL INTERSECTION of A GRADE THREE GoRENSTEIN IDEAL.}

Definition 10.1. Let $R$ be a commutative noetherian ring, $I$ an ideal in $R, f$ an integer with $f \geq$ ht $I, A$ a proper subideal of $I$ which can be generated by $f$ elements, and $J$ the ideal $A: I$. If ht $J \geq f$, then $J$ is called an $f$-residual intersection of $I$. If, furthermore, $I_{P}=A_{P}$ for all prime ideals $P$ of $R$ with $I \subseteq P$ and ht $P \leq f$, then $J$ is called a geometric $f$-residual intersection of $I$.

The main result of this section is

Theorem 10.2. Let I be a grade three Gorenstein ideal in the Cohen-Macaulay local ring $(R, \mathfrak{m}, k)$; let $J=(A: I)$ be an $f$-residual intersection; and let $t$ denote $\mu(I / A)$. If either

(i) the ring $R$ is Gorenstein, or else,

(ii) the residual intersection $J=(A: I)$ is geometric,

then there is an almost alternating matrix $\rho^{\prime}$ with entries in $\mathfrak{m}$ of shape $(t, f)$ such that the following statements hold.

(a) If $J\left(\rho^{\prime}\right)$ is the ideal defined in (4.10), then $J=J\left(\rho^{\prime}\right)$.

(b) The complex $\mathfrak{D}^{0}\left(\rho^{\prime}\right)$ is the minimal free $R$-resolution of $R / J$.

(c) If $1 \leq q \leq f-1$, then $\mathfrak{D}^{q}\left(\rho^{\prime}\right)$ is the minimal $R$-free resolution of $S_{q}(I / A)$. If, in addition, the residual intersection is geometric, then $S_{q}(I / A) \cong \bar{I}^{q}$, where $\bar{I}$ represents the ideal $(I+J) / J$ of $R / J$.

Our proof of Theorem 10.2 will involve deforming the given residual intersection into a "generic" residual intersection, observing that the result is true at the generic level, and then specializing back to the original situation. We begin by giving some hypotheses under which a specialization map $\chi$ preserves the colon operation:

$$
\chi(\widetilde{A}: \widetilde{I})=(\chi(\widetilde{A}): \chi(\widetilde{I})) .
$$

Lemma 10.4 is not the ultimate result along these lines, but it is adequate for our purposes. Recall from [29] that an ideal is said to be strongly Cohen-Macaulay if the Koszul homology modules of some (and hence any) set of generators of $I$ are Cohen-Macaulay modules. Following the lead of [1], we say that the $R$-ideal $I$ satisfies the property

$$
\left(G_{f}\right)
$$

if $\mu\left(I_{P}\right) \leq$ ht $P$ for all prime ideals $P$ of $R$ with $I \subseteq P$ and ht $P \leq f-1$. The ideal $I$ satisfies $\left(G_{\infty}\right)$ if $I$ satisfies $\left(G_{f}\right)$ for all $f$.

Lemma 10.4. (Huneke-Ulrich) Let $\widetilde{J}=(\widetilde{A}: \widetilde{I})$ be a geometric $f$-residual intersection in a local Cohen-Macaulay ring $\widetilde{R}$. Suppose that the $\widetilde{R}-i d e a l \widetilde{J}$ has height $f$ and that the ring $\widetilde{R} / \widetilde{J}$ is Cohen-Macaulay. Let $\mathbf{x}=x_{1}, \ldots, x_{k}$ be a sequence of elements from $\widetilde{R}$ which forms a regular sequence on both $\widetilde{R}$ and $\widetilde{R} / \widetilde{I}$; let $R$ be the ring $\widetilde{R} /(\mathbf{x})$; and let $\chi$ be the natural map $\chi: \widetilde{R} \rightarrow(\widetilde{R} /(\mathbf{x}))=R$. Suppose that $J=(\chi(\widetilde{A}): \chi(\widetilde{I}))$ is an $f$-residual intersection in $R$. If either

(i) the ring $\widetilde{R}$ is Gorenstein, the $\widetilde{R}$-ideal $\widetilde{I}$ is strongly Cohen-Macaulay and 
satisfies the condition $\left(G_{f}\right)$, and the canonical module of $\widetilde{R} / \widetilde{J}$ is isomorphic to

$$
\frac{\widetilde{I}^{n}+\widetilde{J}}{\widetilde{J}}
$$

for some integer $n>0$, or else,

(ii) the residual intersection $J=(\chi(\widetilde{A}): \chi(\widetilde{I}))$ is geometric,

then $J=\chi(\widetilde{J}), R / J$ is a Cohen-Macaulay ring, and the height of the $R$-ideal $J$ is precisely $f$.

Proof. All references in this proof are to [32].

(i) Theorem 1.5 yields that $\operatorname{depth}(\widetilde{R} / \widetilde{A})=\operatorname{dim} \widetilde{R}-f$. It follows from Proposition 3.2 (i) that $\mathbf{x}$ is a regular sequence on $\widetilde{R} / \widetilde{A}$. If $f>$ ht $\widetilde{I}$, then the result is Theorem 3.7. If $f=\mathrm{ht} \widetilde{I}$, then the result is [30, Lemma 2.12].

(ii) The result is Proposition 3.2 (ii).

The next result is the global version of Theorem 10.2.

Theorem 10.5. Let $R$ be a Cohen-Macaulay ring, $g \geq 3$ an odd integer, and $X$ a $g \times g$ alternating matrix over $R$. Assume that the ideal $I=\operatorname{Pf}_{g-1}(X)$ has grade three and that $J$ is an $f$-residual intersection of $I$. If either

(i) the ring $R$ is Gorenstein, or else,

(ii) the ideal $J$ is a geometric residual intersection of $I$,

then there is an almost alternating map $\rho$ of shape $(g, f)$ over $R$ such that the following statements hold.

(a) If $J(\rho)$ is the ideal defined in (4.10), then $J=J(\rho)$.

(b) The complex $\mathfrak{D}^{0}(\rho)$ is a resolution of $R / J$ by free $R$-modules.

(c) If $1 \leq q \leq f-1$, then the complex $\mathfrak{D}^{q}(\rho)$ is a free $R$-resolution of $S_{q}(I / A)$. If, in addition, the residual intersection is geometric, then $S_{q}(I / A) \cong \bar{I}^{q}$, where $\bar{I}$ represents the ideal $(I+J) / J$ of $R / J$.

Proof. The ideal $I$ is generated by the entries of $\mathbf{x}=\left[X_{\langle 1\rangle}, \ldots, X_{\langle g\rangle}\right]$ for $X_{\langle j\rangle}$ defined above (4.16). The ideal $J$ is defined to be $(A: I)$ for some $f$-generated ideal $A$. There exists a $g \times f$ matrix $Y$ so that $A=I_{1}(\mathrm{x} Y)$. Let $\rho$ be the almost alternating matrix $\left[\begin{array}{ll}X & Y\end{array}\right]$. If $A(\rho)$ and $I(\rho)$ are the ideals defined in (4.10), then observe that $A=A(\rho)$ and $I=I(\rho)$. Part (d.iii) of Proposition 4.13 shows that $J(\rho) \subseteq(A: I)=J$. The first assertion of the present theorem is equivalent to the statement:

$$
J(\rho)=J
$$

Assume, for the time being, that equality holds in (10.6). The rest of the theorem follows quickly. Indeed, Theorem 8.3 (b.i) (by way of Corollary 5.5) yields that $\mathfrak{D}^{q}$ is acyclic for $-1 \leq q \leq f-1$. Assertion (b) is thereby established. If $q \geq 1$, then Proposition 4.13 shows that $H_{0}\left(\mathfrak{D}^{q}\right) \cong S_{q}(I / A)$. If $J$ is a geometric $f$-residual intersection of $I$, then ht $(I+J) \geq f+1$. Hence, for $1 \leq q \leq f-1$, $H_{0}\left(\mathfrak{D}^{q}\right)$ is a torsion-free $(R / J)$-module of rank one by Theorem $8.3(\mathrm{~b})$ and Observation 5.12 (b). Furthermore, $\bar{I}$ is an ideal of positive grade. Consequently, we 
may apply Observation 1.27 in order to see that the natural map $S_{q}(\bar{I}) \rightarrow \bar{I}^{q}$ is an isomorphism.

To prove equality in (10.6), it suffices to prove equality locally at each maximal ideal of $R$. After a change of notation, we may assume that $(R, \mathfrak{m})$ is a local ring. Let $\widetilde{\rho}=\left[\begin{array}{ll}\widetilde{X} & \widetilde{Y}\end{array}\right]$ be a generic almost alternating matrix of shape $(g, f), Z$ the ideal $\left(\widetilde{x}_{i j}-x_{i j}, \widetilde{y}_{i j}-y_{i j}\right)$ of the polynomial $\operatorname{ring} R[\widetilde{X}, \widetilde{Y}], \mathfrak{M}$ the maximal ideal $(\mathfrak{m}, Z)$ of $R[\widetilde{X}, \widetilde{Y}]$, and $\widetilde{R}$ the $\operatorname{ring} R[\widetilde{X}, \widetilde{Y}]_{\mathfrak{M}}$. Form the ideals $\widetilde{J}=J(\widetilde{\rho}), \widetilde{I}=I(\widetilde{\rho})$, and $\widetilde{A}=A(\widetilde{\rho})$ as described in Definition 4.10. The note following Corollary 8.9 shows that

$$
(\widetilde{A}: \widetilde{I})=\widetilde{J}
$$

is a geometric $f$-residual intersection. Let $\chi: \widetilde{R} \rightarrow(\widetilde{R} / Z)=R$ be the natural map. Observe, using the ideas of $(8.5)$, that $\chi(\widetilde{A})=A, \chi(\widetilde{I})=I$, and $\chi(\widetilde{J})=$ $J(\rho)$. It is clear, from dimension considerations, that the listed generators of $Z$ form a regular sequence on both $\widetilde{R}$ and $\widetilde{R} / \widetilde{I}$. As soon as we verify that the rest of the hypotheses of Lemma 10.4 are satisfied, then we will know that the equation of (10.3) holds; and therefore, we can combine all of the above equations in order to conclude that

$$
J(\rho)=\chi(\widetilde{J})=\chi(\widetilde{A}: \widetilde{I})=(\chi(\widetilde{A}): \chi(\widetilde{I}))=(A: I)=J .
$$

We know, from Theorem 7.3 , that $\widetilde{J}$ is a perfect $\widetilde{R}$-ideal of grade $f$; and hence, $\widetilde{R} / \widetilde{J}$ is a Cohen-Macaulay ring. The proof is complete if hypothesis (ii) is in effect. Henceforth, we assume that $R$ is a Gorenstein ring. Theorem 7.3 also shows that

$$
\operatorname{Ext}_{\widetilde{R}}^{f}(\widetilde{R} / \widetilde{J}, \widetilde{R})=H_{0}\left(\mathfrak{D}^{f-2}\right)=\frac{\widetilde{I}^{f-2}+\widetilde{J}}{\widetilde{J}}
$$

hence, the canonical module of $\widetilde{R} / \widetilde{J}$ has the required form. The main theorem of [28] ensures that the ideal $\widetilde{I}$ is strongly Cohen-Macaulay. It is easy to verify that $\widetilde{I}$ satisfies the condition $\left(G_{f}\right)$.

Proof of Theorem 10.2. Let $g$ denote $\mu(I)$. The main theorem of [14] guarantees that there is a $g \times g$ alternating matrix $X$, with entries in $\mathfrak{m}$, such that $\operatorname{Pf}_{g-1}(X)=I$. We apply Theorem 10.5 to find an almost alternating matrix $\rho=\left[\begin{array}{ll}X & Y\end{array}\right]$ of shape $(g, f)$ with $J=J(\rho)$. Every entry of $X$ is in $\mathfrak{m}$; consequently, the hypothesis $\mu(I / A)=t$ ensures that the rank of $\rho \otimes k$ is $g-t$. According to Lemma 5.4, there is an almost alternating matrix $\rho^{\prime}$ of shape $(t, f)$ with $J\left(\rho^{\prime}\right)=J(\rho)=J$. It is clear that every entry of $\rho^{\prime}$ is in $\mathfrak{m}$. Assertion (a) is established. The grade of $J$ is $f$, so Theorem 8.3 (b.i) shows that $\mathfrak{D}^{q}\left(\rho^{\prime}\right)$ is acyclic for $-1 \leq q \leq f-1$. Since the entries of $\rho^{\prime}$ are in $\mathfrak{m}$, it follows from Observation 4.28 that $\mathfrak{D}^{q}\left(\rho^{\prime}\right)$ is a minimal resolution for $q$ in the above range. Parts (b) and (c) follow from Lemma 5.4 (c) and Theorem 10.5.

The next result says that if $I$ has enough generators, then the minimal number of generators of an $f$-residual intersection of $I$ depends only on $f$; it does not depend on $\mu(I)$ or on $\mu(I / A)$. If $f=3$, then this phenomenon is well-known, because a link $J$ of a grade three Gorenstein ideal has at most four generators; furthermore, if $\mu(J) \leq 3$, then $I$ is a complete intersection by [39]. 
Corollary 10.7. Let I be a grade three Gorenstein ideal in a Cohen-Macaulay local ring $R$; and let $J$ be an $f$-residual intersection of $I$. If either

(i) the ring $R$ is Gorenstein, or else

(ii) the ideal $J$ is a geometric residual intersection of $I$, then

$$
\mu(J) \leq 2^{f-1}
$$

furthermore, if $2 f-1 \leq \mu(I)$, then equality holds in (10.8).

Proof. The ideal $J$ is equal to $A: I$ for some $f$-generated ideal $A$. Let $t=\mu(I / A)$. We have seen in Theorem 10.2 that there is an almost alternating map $\rho^{\prime}$ of shape $(t, f)$ so that $\mathfrak{D}^{0}\left(\rho^{\prime}\right)$ is a minimal resolution of $R / J$. It follows that $\mu(J)$ is equal to the rank of $Q_{0}=Q_{0}\left(\rho^{\prime}\right)$. We know, from (2.16), that $Q_{0}$ is equal to $\oplus_{r} \bigwedge^{r} F$, where the sum is taken over all integers $r$ with $t+r$ even and $2-t \leq r \leq t$. Thus,

$$
\mu(J) \leq \sum_{\text {all } r \text { of fixed parity }}\left(\begin{array}{l}
f \\
r
\end{array}\right)=2^{f-1} ;
$$

and equality is obtained if $f-1 \leq t$. On the other hand, if $2 f-1 \leq \mu(I)$, then

$$
f-1=(2 f-1)-f \leq \mu(I)-f \leq \mu(I) \leq \mu(I)-\mu(A) \leq \mu(I / A)=t .
$$

Acknowledgement. This paper was prepared while the first author was on sabbatical. He appreciates the hospitality he received at Michigan State University. The calculations that lead us to the complexes $\left\{\mathfrak{D}^{q}\right\}$ were made using the MACAULAY program of D. Bayer and $M$. Stillman on the computers of the Departments of Mathematics and Computer Science at the University of South Carolina and the Department of Mathematics at Michigan State University. 


\section{REFERENCES}

1. M. Artin and M. Nagata, Residual intersections in Cohen-Macaulay rings, J. Math. Kyoto Univ. 12 (1972), 307-323.

2. L. Avramov, A class of factorial domains, Serdica 5 (1979), 378-379.

3. L. Avramov, A. Kustin, and M. Miller, Poincaré series of modules over local rings of small embedding codepth or small linking number, J. Alg. 118 (1988), 162-204.

4. G. Boffi and R. Sánchez, On the square of the pfaffian ideal, preprint (1989).

5. M. Brodmann, The asymptotic nature of the analytic spread, Math. Proc. Camb. Phil. Soc. 86 (1979), 35-39.

6. W. Bruns, Die Divisorenklassengruppe der Restklassenringe von Polynomringen nach Determinantenidealen, Revue Roumaine Math. Pur. Appl. 20 (1975), 1109-1111.

7. W. Bruns, A. Kustin, and M. Miller, The resolution of the generic residual intersection of a complete intersection, J. Alg. 128 (1990), 214-239.

8. W. Bruns and U. Vetter, Length formulas for the local cohomology of exterior powers, Math. Z. 191 (1986), 145-158.

9. W. Bruns and U. Vetter, Determinantal rings, Lecture Notes in Mathematics 1327, Springer Verlag, Berlin Heidelberg New York, 1988.

10. D. Buchsbaum, A generalized Koszul complex. I, Trans. Amer. Math. Soc. 111 (1964), 183196.

11. D. Buchsbaum and D. Eisenbud, What makes a complex exact?, J. Alg. 25 (1973), 259-268.

12. D. Buchsbaum and D. Eisenbud, Remarks on ideals and resolutions, Symp. Math. 11 (1973), 193-204.

13. D. Buchsbaum and D. Eisenbud, Generic free resolutions and a family of generically perfect ideals, Advances Math. 18 (1975), 245-301.

14. D. Buchsbaum and D. Eisenbud, Algebra structures for finite free resolutions, and some structure theorems for ideals of codimension 3, Amer. J. Math. 99 (1977), 447-485.

15. D. Buchsbaum and D. Rim, A generalized Koszul complex. II, Trans. Amer. Math. Soc. 111 (1964), 197-224.

16. R.-O. Buchweitz, Contributions à la théorie des singularités, Thesis, L'Université Paris VII, 1981.

17. R.-O. Buchweitz and B. Ulrich, Homological properties which are invariant under linkage, preprint.

18. L. Burch, Codimension and analytic spread, Math. Proc. Camb. Phil. Soc. 72 (1972), 369-373.

19. C. Day, Ring around the poset: an introduction to Algebras with Straightening Law, M. A. thesis, University of South Carolina, 1989.

20. J. Eagon and D. Northcott, Ideals defined by matrices and a certain complex associated with them, Proc. Roy. Soc. London Ser. A 269 (1962), 188-204.

21. R. Fossum, The divisor class group of a Krull domain, Springer Verlag, Berlin Heidelberg New York, 1973.

22. J. Herzog, Deformationen von Cohen-Macaulay Algebren, J. reine angew. Math. 318 (1980), 83-105.

23. J. Herzog and B. Ulrich, Self-linked curve singularities, preprint (1989).

24. M. Hochster, Properties of noetherian rings which are stable under general grade reduction, Arch. Math. 24 (1973), 393-396.

25. M. Hochster, Topics in the homological theory of modules over commutative rings, CBMS Regional Conf. Ser. in Math., no. 24, Amer. Math. Soc., Providence, RI, 1975.

26. M. Hochster and J. Eagon, Cohen-Macaulay rings, invariant theory, and the generic perfection of determinantal loci, Amer. J. Math. 93 (1971), 1020-1058.

27. C. Huneke, The theory of $d$-sequences and powers of ideals, Advances Math. 46 (1982), 249-279.

28. C. Huneke, Linkage and the Koszul homology of ideals, Amer. J. Math. 104 (1982), 10431062 .

29. C. Huneke, Strongly Cohen-Macaulay schemes and residual intersections, Trans. Amer. Math. Soc. 277 (1983), 739-763. 
30. C. Huneke and B. Ulrich, Divisor class groups and deformations, Amer. J. Math. 107 (1985), 1265-1303.

31. C. Huneke and B. Ulrich, The structure of linkage, Annals of Math. 126 (1987), 277-334.

32. C. Huneke and B. Ulrich, Residual intersections, J. reine angew. Math. 390 (1988), 1-20.

33. C. Huneke and B. Ulrich, Powers of licci ideals, Commutative Algebra, Mathematical Sciences Research Institute Publications 15, Springer Verlag, Berlin Heidelberg New York, 1989, pp. 339-346.

34. C. Huneke and B. Ulrich, Generic residual intersections, preprint (1989).

35. T. Józefiak, P. Pragacz, and J. Weyman, Resolutions of determinantal varieties and tensor complex associated with symmetric and antisymmetric matrices, Astérisque 87/88 (1981), 109-189.

36. S. Kim, Projective resolutions of generic order ideals, Ph. D. thesis, University of Illinois, Urbana, 1988.

37. D. Kirby, A sequence of complexes associated with a matrix, J. London Math. Soc. (2) 7 (1973), 523-530.

38. H. Kleppe and D. Laksov, The algebraic structure and deformation of Pfaffian schemes, J. Alg. 64 (1980), 167-189.

39. E. Kunz, Almost complete intersections are not Gorenstein rings, J. Alg. 28 (1974), 111-115.

40. A. Kustin, The minimal free resolution of the Huneke-Ulrich deviation two Gorenstein ideals, J. Alg. 100 (1986), 265-304.

41. A. Kustin, M. Miller, and B. Ulrich, Generating a residual intersection, J. Alg. (to appear).

42. A. Kustin and B. Ulrich, If the socle fits, J. Alg. (to appear).

43. V. Marinov, Perfection of ideals generated by the pfaffians of an alternating matrix, C.R. Acad. Bulg. Sci. 31 (1979), 561-563.

44. V. Marinov, Perfection of ideals generated by the pfaffians of an alternating matrix, Serdica 9 (1983), 31-42; 122-131.

45. H. Matsumura, Commutative algebra, 2nd ed., Benjamin/Cummings, Reading, MA, 1980.

46. J. Migliore, Geometric invariants for liaison of space curves, J. Alg. 99 (1986), 548-572.

47. C. Peskine and L. Szpiro, Liaison des variétés algébriques I, Invent. Math. 26 (1974), 271-302.

48. A. Rao, Liaison among curves in $\mathbb{P}^{3}$, Invent. Math. 50 (1979), 205-217.

49. P. Schwartau, Liaison addition and monomial ideals, Ph.D. thesis, Brandeis University, Waltham, MA, 1982.

50. H. Srinivasan, Decomposition formulas for pfaffians, preprint (1988).

51. H. Srinivasan, Minimal algebra resolutions for cyclic modules defined by Huneke-Ulrich ideals, preprint (1989).

Department of Mathematics, University of South Carolina, Columbia, SC 29208 E-mail address: n410123 at univscvm.bitnet

Department of Mathematics, Michigan State University, East Lansing, Mi 48824

E-mail address: 21144bfu at msu.bitnet 